University of Tennessee Health Science Center

UTHSC Digital Commons

\title{
Overcoming Acquired Resistance to BRAF Inhibitors by Novel Synergistic Drug Combination and Discovery of Novel Smac Mimetics as Selective Survivin Inhibitors
}

Jin Wang

University of Tennessee Health Science Center

Follow this and additional works at: https://dc.uthsc.edu/dissertations

Part of the Medicinal and Pharmaceutical Chemistry Commons, and the Pharmaceutics and Drug Design Commons

\section{Recommended Citation}

Wang, Jin , "Overcoming Acquired Resistance to BRAF Inhibitors by Novel Synergistic Drug Combination and Discovery of Novel Smac Mimetics as Selective Survivin Inhibitors" (2015). Theses and Dissertations (ETD). Paper 282. http://dx.doi.org/10.21007/etd.cghs.2015.0341. 


\title{
Overcoming Acquired Resistance to BRAF Inhibitors by Novel Synergistic Drug Combination and Discovery of Novel Smac Mimetics as Selective Survivin Inhibitors
}

\begin{abstract}
The first part (Chapter 1 and 2) of this dissertation presents a novel combination study of melanoma therapy. Acquired clinical resistance to vemurafenib, a selective BRAFV600E inhibitor, arises frequently after short term chemotherapy. Since the inhibitions of targets in the RAFMEK-ERK pathway result in G0/ G1 cell cycle arrest, vemurafenib-resistant cancer cells are expected to escape this cell cycle arrest and progress to subsequent $\mathrm{G} 2 / \mathrm{M}$ phase. We hypothesized that a combined therapy using vemurafenib with a G2/M phase blocking agent will trap resistant cells and overcome vemurafenib resistance. To test this hypothesis, we first determined the combination index $(\mathrm{Cl})$ values of our novel tubulin inhibitor $\mathrm{ABI}-274$ and vemurafenib on parental human A375 and MDA-MB-435 melanoma cell lines to be 0.32 and 0.1 , respectively, suggesting strong synergy for the combination. We then developed an A375RF21 subline with significant acquired resistance to vemurafenib and confirmed the strong synergistic effect. Next we studied the potential mechanisms of overcoming vemurafenib resistance. Flow cytometry confirmed that the combination of ABI-274 and vemurafenib synergistically arrested cells in G1/G2/M phase, and significantly increased apoptosis in both parental A375 and the vemurafenib-resistant A375RF21 cells. Western blot analysis revealed that the combination treatment effectively reduced the level of phosphorylated and total AKT, activated the apoptosis cascade, and increased cleaved caspase- 3 and cleaved PARP, but had no significant influence on the level of ERK phosphorylation. Finally, in vivo coadministration of vemurafenib with $\mathrm{ABI}-274$ showed strong synergistic efficacy in the vemurafenibresistant xenograft model in nude mice. Overall, these results offer a rational combination strategy to significantly enhance the therapeutic benefit in melanoma patients who inevitably become resistant to current BRAF inhibition therapy.
\end{abstract}

The second part (Chapter 3 to 5 ) of this dissertation focuses on the discovery of a series of small molecule survivin inhibitors. Inhibitors of apoptosis (IAP) proteins are widely considered as promising cancer drug targets, especially for drug-resistant tumors. Mimicking the IAP-binding motif of second mitochondria-derived activator of caspases (Smac) is a rational strategy to design potential IAP inhibitors. In this report, we used the bioactive conformation of AVPI tetrapeptide in the N-terminus of Smac as a template and performed a shape-based virtual screening against a drug-like compound library to identify novel IAP inhibitors. Top hits were subsequently docked to available IAP crystal structures as a secondary screening followed by validation using in vitro biological assays. Four novel hit compounds were identified that potently inhibited cell growth in two human melanoma (A375 and M14) and two human prostate (PC-3 and DU145) cancer cell lines. The best compound, UC-112, has IC50 values ranging from 0.7 to $3.4 \mu \mathrm{M}$. UC-112 also potently inhibits the growth of P-glycoprotein (Pgp) overexpressed multidrug-resistant cancer cells, strongly activates capase-3/7 and caspase- 9 activities, and selectively down-regulates survivin level at a concentration as low as $1 \mu \mathrm{M}$. Co-incubation of UC-112 with a known proteasome inhibitor (MG132) rescued survivin inhibition, consistent with the anticipated mechanism of action for UC-112. As a single agent, UC-112 strongly inhibits tumor growth and reduces both XIAP and survivin levels in an $\mathrm{A} 375$ human melanoma xenograft model in vivo. Three analogs generated from UC-112 structural modification along with template compound UC-112 were submitted to $\mathrm{NCl}-60$ cancer cell line screening. The results indicated that structural modification of UC-112 to give our best compound MX106 has improved activity by four fold $(2.2 \mu \mathrm{M}$ for UC-112 vs. $0.5 \mu \mathrm{M}$ for MX106, average GI50 values over all cancer cell lines in the $\mathrm{NCl}-60$ panel). Western blot analyses demonstrated the new compounds maintained high selectivity for survivin inhibition over other members in the inhibitiors of apoptosis protein family. When tested in an A375 human melanoma xenograft model, the most active compound MX106 effectively suppressed tumor growth and strongly induced cancer cell apoptosis in tumor tissues. 
Taken together, this novel scaffold is promising for the development of selective survivin inhibitors as potential anticancer agents.

\section{Document Type}

Dissertation

Degree Name

Doctor of Philosophy (PhD)

\section{Program}

Pharmaceutical Sciences

\section{Research Advisor}

Wei Li, Ph.D.

\section{Keywords}

BRAF inhibitor, combination therapy, inhibitor of apoptosis, melanoma, survivin inhibitor, tubulin inhibitor

\section{Subject Categories}

Medicinal and Pharmaceutical Chemistry | Medicine and Health Sciences | Pharmaceutics and Drug Design | Pharmacy and Pharmaceutical Sciences 
OVERCOMING ACQUIRED RESISTANCE TO BRAF INHIBITORS BY NOVEL SYNERGISTIC DRUG COMBINATION AND DISCOVERY OF NOVEL SMAC MIMETICS AS SELECTIVE SURVIVIN INHIBITORS

\author{
A Dissertation \\ Presented for \\ The Graduate Studies Council \\ The University of Tennessee \\ Health Science Center
}

\begin{abstract}
In Partial Fulfillment
Of the Requirements for the Degree

Doctor of Philosophy

From The University of Tennessee
\end{abstract}

\title{
By
}

Jin Wang

August 2015 
Chapter 1 (C) 2014 by InTech

Portions of Chapter 2 (C) 2014 by American Association for Cancer Research

Portions of Chapter 4 (C) 2014 by the American Society for Pharmacology and

Experimental Therapeutics

Portions of Chapter 5 (C) 2015 by Public Library of Science

All other material (C) 2015 by Jin Wang

All rights reserved. 


\section{ACKNOWLEDGEMENTS}

I'm heartily grateful to my research advisor, Dr. Wei Li, who tutored, inspired and supported me to conduct this research throughout my $\mathrm{Ph} . \mathrm{D}$ years. His insightful minds, patient guidance and diligent working attitude have leaded me through the darkness of my study and will always encourage me in the future time as a scholar.

I would like to take this opportunity to extend my gratitude to my committee, Drs. Miller, Meibohm, Slominski and Park for their supervision, comments, and assistance. I want to especially thank Dr. Duane D. Miller and his people for their help, discussion and encouragement of my routine work in our joint group meetings.

I'm sincerely thankful to Dr. Jianjun Chen, Dr. Yan Lu and Mr. Min Xiao, who synthesized the compounds for my dissertation studies. And I appreciate the training of pathology study from Dr. Andrzej T. Smominski's group and pharmacokinetics study from Dr. Bernd Meibohm's group. I thank Dr. Sunjoo Ahn, Dr. Narayanan Ramesh and Dr. Zorica Janjetovic for their great assistance in my development of biological assays. I thank Dr. David Hamilton and his people who generously give their time and expertise to better my animal work. I thank Dr. Jianjun Chen, Zongtao Lin, Ashit Trivedi and Dr. Dejian Ma for their hands-on training and help in LC/MS/MS. Meifeng Lu and Dr. Dan Rosson kindly provided technical support to my research.

I acknowledge the financial support of the Alma and Hal Reagan Fellowship for the academic years 2012-2014. And I thank the College of Graduate Health Sciences in the University of Tennessee Health Science Center for awarding me with this fellowship as well.

My deepest thank to all the friends, colleagues, and mentors who accompanied, advised, and supported my Ph.D life over the years. The list is long, but I cherish every moment shared with them.

I'm highly indebted to my parents, my husband and my daughter. Their neverending and unconditional love is the greatest motivation in my way to be a better self. 


\begin{abstract}
The first part (Chapter 1 and 2) of this dissertation presents a novel combination study of melanoma therapy. Acquired clinical resistance to vemurafenib, a selective $\mathrm{BRAF}^{\mathrm{V} 600 \mathrm{E}}$ inhibitor, arises frequently after short term chemotherapy. Since the inhibitions of targets in the RAF-MEK-ERK pathway result in $\mathrm{G}_{0} / \mathrm{G}_{1}$ cell cycle arrest, vemurafenib-resistant cancer cells are expected to escape this cell cycle arrest and progress to subsequent $\mathrm{G}_{2} / \mathrm{M}$ phase. We hypothesized that a combined therapy using vemurafenib with a $\mathrm{G}_{2} / \mathrm{M}$ phase blocking agent will trap resistant cells and overcome vemurafenib resistance. To test this hypothesis, we first determined the combination index (CI) values of our novel tubulin inhibitor ABI-274 and vemurafenib on parental human A375 and MDA-MB-435 melanoma cell lines to be 0.32 and 0.1, respectively, suggesting strong synergy for the combination. We then developed an A375RF21 subline with significant acquired resistance to vemurafenib and confirmed the strong synergistic effect. Next we studied the potential mechanisms of overcoming vemurafenib resistance. Flow cytometry confirmed that the combination of ABI-274 and vemurafenib synergistically arrested cells in $\mathrm{G}_{1} / \mathrm{G}_{2} / \mathrm{M}$ phase, and significantly increased apoptosis in both parental A375 and the vemurafenib-resistant A375RF21 cells. Western blot analysis revealed that the combination treatment effectively reduced the level of phosphorylated and total AKT, activated the apoptosis cascade, and increased cleaved caspase- 3 and cleaved PARP, but had no significant influence on the level of ERK phosphorylation. Finally, in vivo co-administration of vemurafenib with ABI-274 showed strong synergistic efficacy in the vemurafenib-resistant xenograft model in nude mice. Overall, these results offer a rational combination strategy to significantly enhance the therapeutic benefit in melanoma patients who inevitably become resistant to current BRAF inhibition therapy.
\end{abstract}

The second part (Chapter 3 to 5) of this dissertation focuses on the discovery of a series of small molecule survivin inhibitors. Inhibitors of apoptosis (IAP) proteins are widely considered as promising cancer drug targets, especially for drug-resistant tumors. Mimicking the IAP-binding motif of second mitochondria-derived activator of caspases (Smac) is a rational strategy to design potential IAP inhibitors. In this report, we used the bioactive conformation of AVPI tetrapeptide in the N-terminus of Smac as a template and performed a shape-based virtual screening against a drug-like compound library to identify novel IAP inhibitors. Top hits were subsequently docked to available IAP crystal structures as a secondary screening followed by validation using in vitro biological assays. Four novel hit compounds were identified that potently inhibited cell growth in two human melanoma (A375 and M14) and two human prostate (PC-3 and DU145) cancer cell lines. The best compound, $\mathrm{UC}-112$, has $\mathrm{IC}_{50}$ values ranging from 0.7 to 3.4 $\mu \mathrm{M}$. UC-112 also potently inhibits the growth of P-glycoprotein (Pgp) overexpressed multidrug-resistant cancer cells, strongly activates capase-3/7 and caspase- 9 activities, and selectively down-regulates survivin level at a concentration as low as $1 \mu \mathrm{M}$. Coincubation of UC-112 with a known proteasome inhibitor (MG132) rescued survivin inhibition, consistent with the anticipated mechanism of action for UC-112. As a single agent, UC-112 strongly inhibits tumor growth and reduces both XIAP and survivin levels 
in an A375 human melanoma xenograft model in vivo. Three analogs generated from UC-112 structural modification along with template compound UC-112 were submitted to NCI-60 cancer cell line screening. The results indicated that structural modification of UC-112 to give our best compound MX106 has improved activity by four fold $(2.2 \mu \mathrm{M}$ for UC-112 vs. $0.5 \mu \mathrm{M}$ for MX106, average $\mathrm{GI}_{50}$ values over all cancer cell lines in the NCI-60 panel).Western blot analyses demonstrated the new compounds maintained high selectivity for survivin inhibition over other members in the inhibitiors of apoptosis protein family. When tested in an A375 human melanoma xenograft model, the most active compound MX106 effectively suppressed tumor growth and strongly induced cancer cell apoptosis in tumor tissues. Taken together, this novel scaffold is promising for the development of selective survivin inhibitors as potential anticancer agents. 


\section{TABLE OF CONTENTS}

\section{CHAPTER 1. EMERGING DRUG COMBINATION APPROACHES IN MELANOMA THERAPY ...................................................................................1}

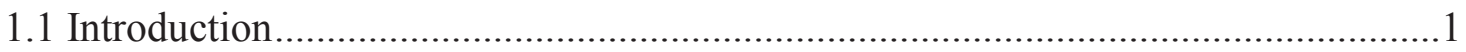

1.2 Combination of Kinase Inhibitors for Melanoma Treatment ...................................1

1.2.1 Combined Inhibitions Targeting Components within the Mitogenactivated Protein Kinase (MAPK) Signaling Pathway .....................................1

1.2.2 Combination Targeted Therapy Using Phosphatidylinositol 3-kinase (PI3K)/AKT/Mammalian Target of Rapamycin (mTOR) Inhibitors ................7

1.2.3 Combining Targeted Therapy with Anti-angiogenic Agents............................8

1.2.4 Combination Therapy Using Targeted Therapy with Versatile

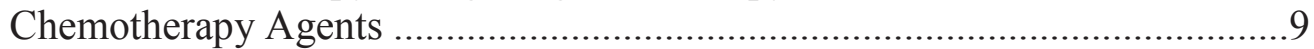

1.3 Combinations Involving Immunotherapy in Melanoma Treatment .........................10

1.3.1 Combined Blockade of Immuno-checkpoints .................................................10

1.3.2 Combined Therapy Inhibiting both Immuno-checkpoint and MAPK

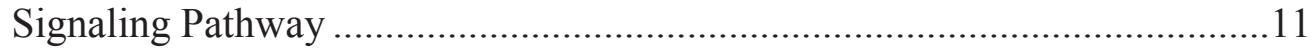

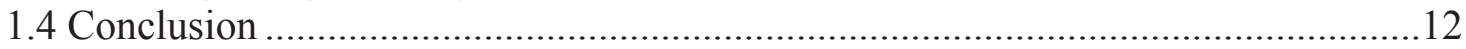

\section{CHAPTER 2. OVERCOMING ACQUIRED RESISTANCE TO BRAF INHIBITORS BY NOVEL SYNERGISTIC DRUG COMBINATION ......................14}

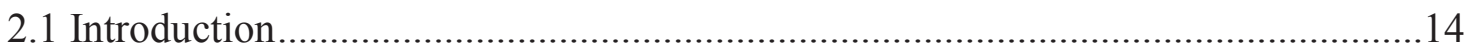

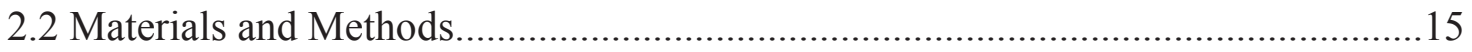

2.2.1 Reagents and Cell Lines.............................................................................

2.2.2 Cell Proliferation and In Vitro Combination Assay ........................................15

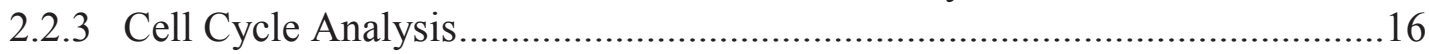

2.2.4 Tubulin Polymerization Assay ....................................................................16

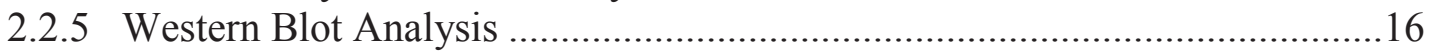

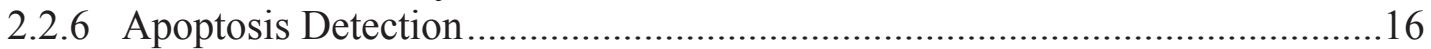

2.2.7 Vemurafenib-resistant Tumor Xenograft and Treatment..................................17

2.2.8 Pathology and Immunohistochemistry (IHC) Analysis ....................................17

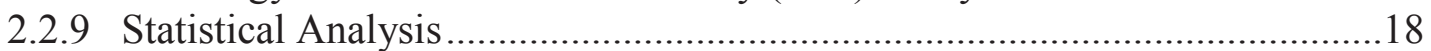

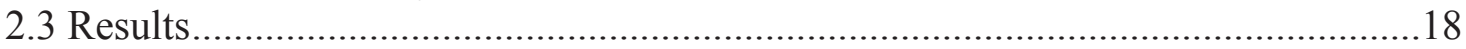

2.3.1 Combination of Vemurafenib with ABI Compound Showed Strong Synergies in Parental BRAF ${ }^{\mathrm{V} 600 \mathrm{E}}$ Mutant Melanoma Cell Lines .....................18

2.3.2 Combination of ABI-231 and Vemurafenib Produced Synergistic Cell Cycle Arrests in Vemurafenib-sensitive A375 Cells......................................18

2.3.3 Combination of ABI-231 and Vemurafenib Affected Related Protein Levels in Vemurafenib-sensitive A375 Cells .................................................18

2.3.4 Establishment of Vemurafenib-resistant A375RF21 Melanoma Subline.......22

2.3.5 Combination of Vemurafenib with ABI-274 Showed Strong Synergies in Vemurafenib-resistant A375RF21 Melanoma Cell Lines ...............................22

2.3.6 Combination of ABI-274 and Vemurafenib Produced Synergistic Cell

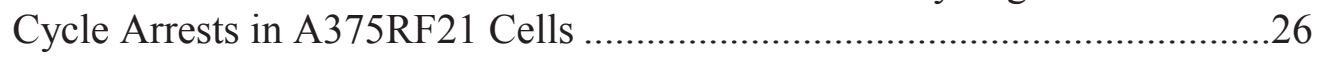


2.3.7 Combination Treatment Induced Significantly Increased Apoptotic and Cell Death in Vemurafenib-resistant Cells ...................................................26

2.3.8 Combination Mitigates Acquired Vemurafenib Resistance by Downregulating pAKT or Total AKT and Activating Apoptosis Cascades ............30

2.3.9 Combination of Vemurafenib and ABI-274 Synergistically Suppresses Vemurafenib-resistant Tumor Growth In Vivo ..............................................34

2.3.10 Establishment of Vemurafenib-resistant MDA-MB-435 VemR Melanoma Subline

2.3.11 Combination of Tubulin Inhibitor with BRAF Inhibitor Showed Synergistic Anti-proliferation Effects in Expanded Panel of Vemurafenib or Trametinib Resistant Cell Lines

\section{CHAPTER 3. LITERATURE REVIEW OF SURVIVIN AND ITS}

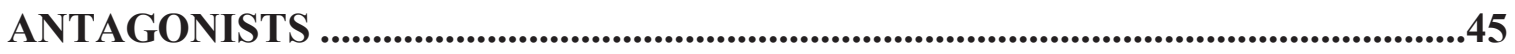

3.1 The Structure and Tissue Distribution of Survivin .............................................45

3.2 The Expression and Regulation of Survivin ..................................................45

3.2.1 Control of Survivin Gene Expression by Transcription Factors ....................47

3.2.2 Post-translational Modification of Survivin................................................47

3.3 The Nodal Functions of Survivin.....................................................................48

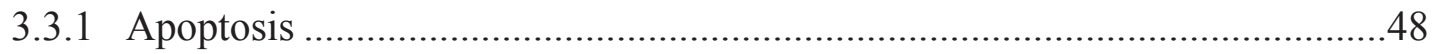

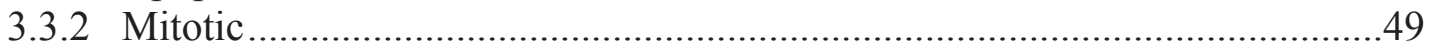

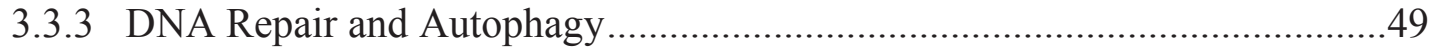

3.4 Current Antagonists of Survivin as Anti-tumor Agents ....................................49

3.4.1 Small Molecule Inhibitors.....................................................................49

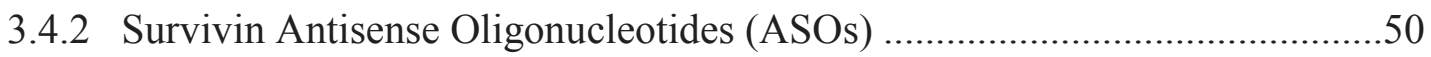

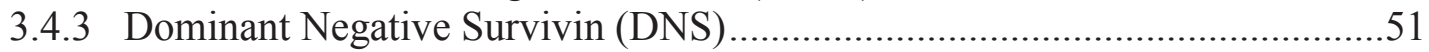

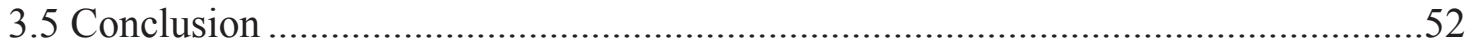

\section{CHAPTER 4. DISCOVERY OF NOVEL SMAC MIMETIC AS SELECTIVE SURVIVIN INHIBITOR .............................................................................................53}

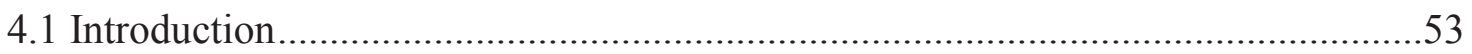

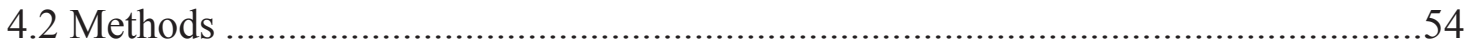

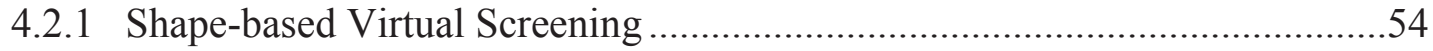

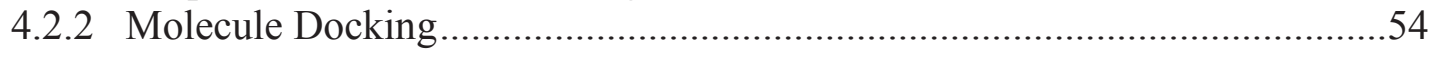

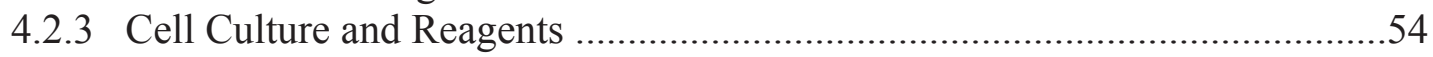

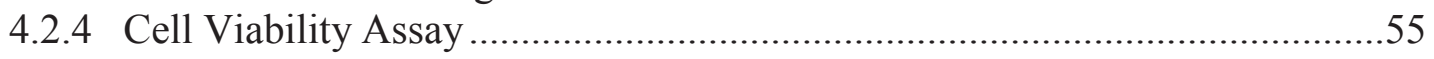

4.2.5 siRNA Silencing Survivin or XIAP Expression ........................................55

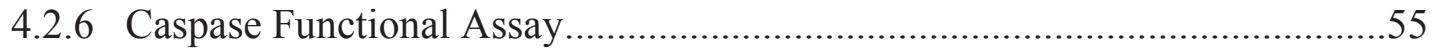

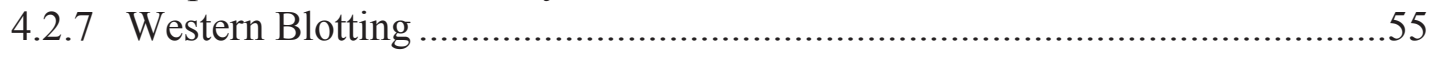

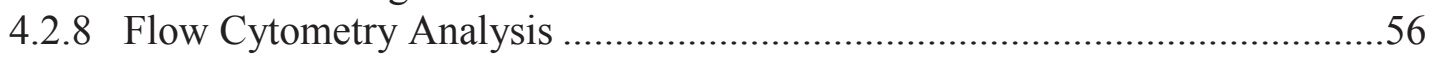

4.2.9 Human Melanoma A375 Tumor Xenograft Model and Treatment .................56

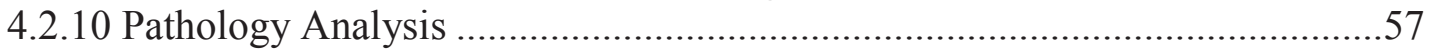

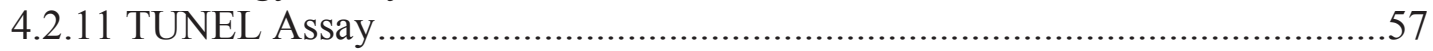

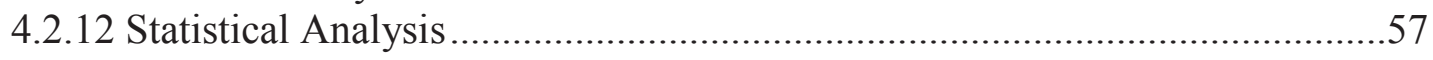

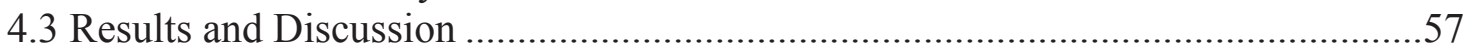


4.3.1 Identification of Initial Hits from Virtual Screening ......................................57

4.3.2 Validation of Virtual Screening Using In Vitro Anti-proliferation Assay.......58

4.3.3 Hit Compounds Increased Caspase Activities Significantly Better than

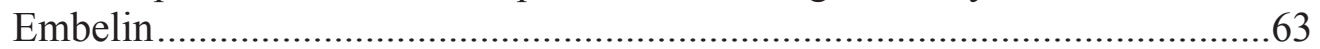

4.3.4 Western Blotting Analyses of IAPs Level Change in Cancer Cells ................63

4.3.5 Proteasome Inhibitor MG132 Rescued the Down-regulated Survivin Levels in Cancer Cells Treated by UC-112 .................................................70

4.3.6 UC-112 Arrested A375 Melanoma Cells in $\mathrm{G}_{1}$ Phase .....................................70

4.3.7 UC-112 Inhibited Tumor Growth in a Melanoma Xenograft Model In

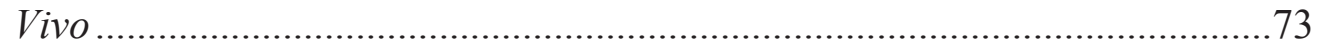

\section{CHAPTER 5. ANTI-CANCER EFFICACY, PHYSIO-CHEMICAL PROPERTY AND TARGET VALIDATION STUDIES OF OPTIMIZED UC-}

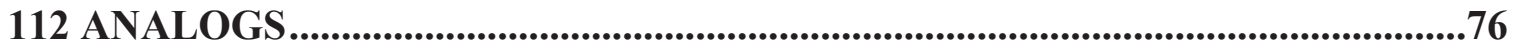

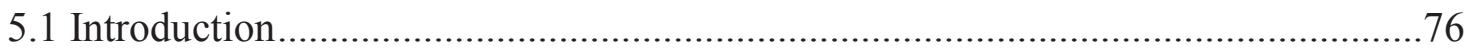

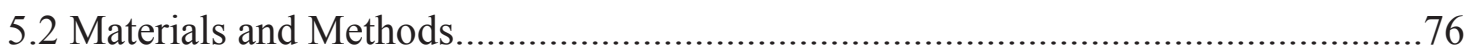

5.2.1 Cell Culture and Cell Viability Assay .........................................................76

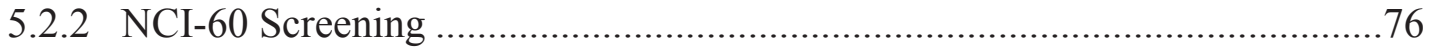

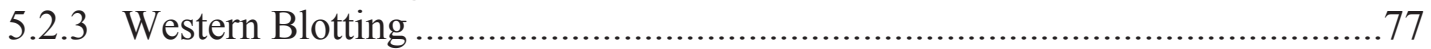

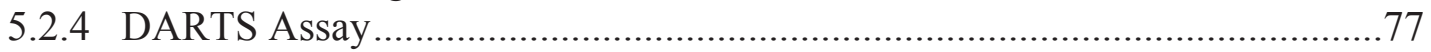

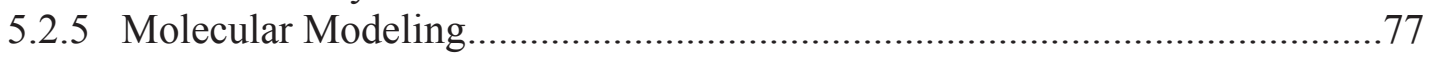

5.2.6 Liver Microsomal Stability Study ……………….....................................78

5.2.7 Plasma Protein Bounding Study ...............................................................78

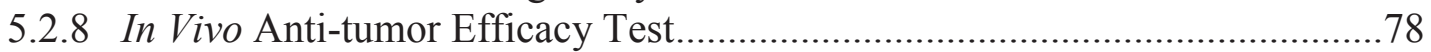

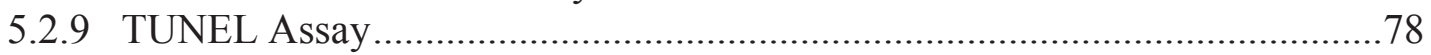

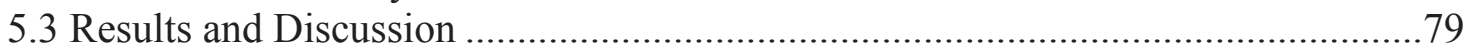

5.3.1 UC-112 Analogs Could Overcome Multidrug Resistance..............................79

5.3.2 UC-112 Analogs Showed Good Anti-proliferation Effects with Selectivity in NCI-60 Cell Line Screening ...................................................................79

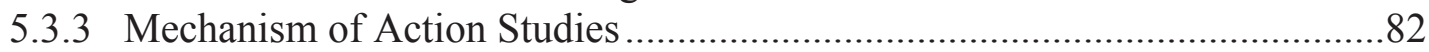

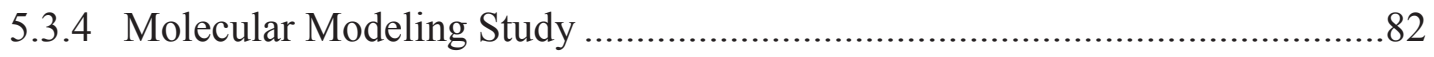

5.3.5 In Vivo Anti-tumor Efficacy Assessment ........................................................86

CHAPTER 6. SUMMARY AND FUTURE DIRECTION ............................................89

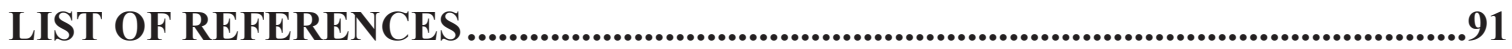

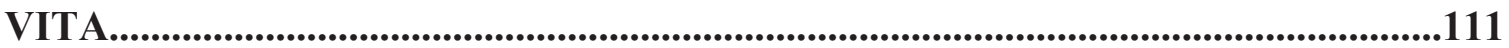




\section{LIST OF TABLES}

Table 2-1. Combination of vemurafenib with tubulin inhibitors showed synergistic effect in the parental BRAF ${ }^{\mathrm{V} 600 \mathrm{E}}$ melanoma cell lines as indicated by $\mathrm{CI}$ values determined at $\mathrm{ED}_{50}$

Table 2-2. Combination of vemurafenib with tubulin inhibitors showed synergistic effect in the vemurafenib-resistant A375RF21 melanoma cell lines

Table 2-3. Tumor growth inhibition (TGI) and tumor weight comparison for in vivo combination of vemurafenib and ABI-274 in the resistant A375RF21 xenograft model $(n=7)$

Table 2-4. Tumor growth inhibition (TGI) and tumor weight comparison for in vivo combination of vemurafenib $(30 \mathrm{mg} / \mathrm{kg})$ and ABI-274 $(15 \mathrm{mg} / \mathrm{kg})$ in the resistant A375RF21 xenograft model $(n=5)$

Table 2-5. Combination of BRAF inhibitors with ABI-274 showed synergistic effect in the expanded panel of vemurafenib-resistant melanoma cell lines

Table 4-1. Growth inhibitory percentages of UC compounds at a concentration of 3 $\mu \mathrm{M}$ or $10 \mu \mathrm{M}$ (MTS assay, $\mathrm{n}=4)$ and $\mathrm{IC}_{50}(\mu \mathrm{M})$ values of $\mathrm{UC}$ compounds in comparison with embelin against cancer cell proliferation (SRB assay, $n=4)$.

Table 4-2. $\quad \mathrm{IC}_{50}(\mu \mathrm{M})$ values of $\mathrm{UC}$ compounds in comparison with embelin against normal cells proliferation (SRB assay, $n=4)$.

Table 4-3. UC-112 overcomes Pgp-mediated drug resistance much better than YM155 $(\mathrm{n}=4)$

Table 5-1. In vitro anti-proliferation activities of UC-112 analogs determined by MTS assay $(n=4)$. 


\section{LIST OF FIGURES}

Figure 1-1. The mechanisms of BRAF inhibitor vemurafenib (Vem) action, toxicity and the interaction between melanoma cells with $\mathrm{T}$ lymphocytes ...................3

Figure 1-2. The mechanisms of acquired resistance to BRAF inhibition...........................

Figure 2-1. Cell cycle analysis of A375 cells incubated with single or combination treatment for $24 \mathrm{~h}(n=3)$

Figure 2-2. Representative images of western blotting results for A375 cell incubated with either single or combination treatment for $24 \mathrm{~h}$

Figure 2-3. Establishment of vemurafenib-resistant A375 melanoma cell line A375RF21 from its parental A375 cell line using chronic selection over 3 months with increasing concentrations of vemurafenib

Figure 2-4. Major vemurafenib resistance mechanisms in A375RF21 cells are the over-expression of PDGFR $\beta$ and the activations of the PI3K-AKT pathway

Figure 2-5. In vitro dose-response curves $(n=5)$ of each combination in A375 and A375RF21 cells.

Figure 2-6. Cell cycle analysis for combination of ABI-274 with vemurafenib ( $n=$ 4)

Figure 2-7. Anti-phospho-histone $\mathrm{H} 3$ and PI (propidium iodide) bivariate staining cell cycle analysis on vemurafenib-resistant A375RF21 cells.

Figure 2-8. Combination of tubulin inhibitors with vemurafenib synergistically increased proportion of cell apoptosis or death in resistant A375RF21 cells.

Figure 2-9. Effect of single agent and combination treatment on purified-protein based tubulin polymerization assay $(n=3)$.

Figure 2-10. Western blot analysis with indicated antibodies on lysate of A375RF21 (A), MDA-MB-435 and WM164 cells (B) after 48h treatment while GAPDH was used as a loading control

Figure 2-11. Western blotting analysis for cell lysates of A375RF21 incubated with indicated treatment for $24 \mathrm{~h}$

Figure 2-12. In vivo combination of vemurafenib and ABI-274 in the resistant A375RF21 xenograft model $(n=7)$. 
Figure 2-13. In vivo combination of high dose vemurafenib $(30 \mathrm{mg} / \mathrm{kg})$ and ABI-274 $(15 \mathrm{mg} / \mathrm{kg})$ in the A375RF21 xenograft model $(n=5)$

Figure 2-14. Establishment of BRAF inhibitor resistant MDA-MB-435 VemR subline with chronically selection in vitro

Figure 3-1. The nodal functions of survivin and targets of survivin inhibitors.

Figure 4-1. In silico similarity alignments of template ligand, Smac N-terminus tetrapeptide AVPI (blue stick), with representative UC compounds (UC222 in brown tube; UC-112 in yellow tube

Figure 4-2. In silico predicted binding poses of 50 hits selected for in vitro activity evaluation docked into AVPI binding sides in different XIAP BIR3Smac complexes

Figure 4-3. Cell growth inhibition percentages of XIAP inhibitor hits on human melanoma A375 and M14 cells at $4 \mu \mathrm{M}$ or $10 \mu \mathrm{M}$ after $48 \mathrm{~h}$ incubation determined by MTS assay $(n=4)$.

Figure 4-4. Relative in vitro caspase activity of human melanoma A375 or human prostate cancer PC-3 cells after treatment with UC compounds or embelin at different concentrations $(n=4)$

Figure 4-5. Compounds UC-112 and UC-222 down-regulate the IAP levels in vitro ....66

Figure 4-6. Lane density quantification data of western blotting analysis results (A375 cells) in (A) Figure 4-5A and (B) Figure 4-5C

Figure 4-7. $\mathrm{IC}_{50}$ values of UC-112 increased in survivin or XIAP gene silenced A375 and PC-3 cells.

Figure 4-8. Proteasome inhibitor MG132 can rescue survivin inhibition by UC-112 ....72

Figure 4-9. UC-112 (20 mg/kg and $40 \mathrm{mg} / \mathrm{kg})$ strongly suppress melanoma tumor growth in an A375 xenograft mouse model ( $n=7$, i.p. injection 5 days per week)

Figure 4-10. Tumor images of in vivo anti-tumor efficacy evaluation of UC-112 (20 $\mathrm{mg} / \mathrm{kg}$ and $40 \mathrm{mg} / \mathrm{kg})$ on A375 xenograft nude mice model $(n=7$, i.p. injection five days per week for three continuous weeks)

Figure 5-1. Average $\mathrm{GI}_{50}$ data for UC-112, compound MX35, MX86 and MX106 tested in NCI-60 anti-proliferative screening....

Figure 5-2. Heat map showing the $\mathrm{GI}_{50}$ values (nM) for $\mathrm{UC}-112$ and three analogs in the NCI-60 screening ..... 
Figure 5-3. Western blotting analysis of MX106 in A375 and PC-3 cell lines.....

Figure 5-4. Representative DARTS results for pronase-digested A375 or M14 cell

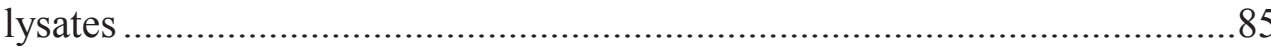

Figure 5-5. Potential binding pose of UC-112 and compound MX106 in the Smac N-terminus tetra-peptide AVPI binding site of survivin crystal structure

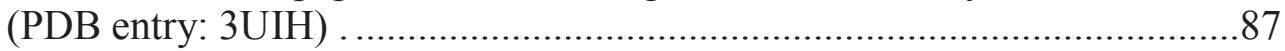

Figure 5-6. In vivo anti-tumor efficacy of compound MX106..................................88 


\section{LIST OF ABBREVIATIONS}

\begin{tabular}{|c|c|}
\hline ABCG2 & ATP-binding cassette sub-family G member 2 \\
\hline AICAR & 5-aminoimidazole-4-carboxamide-1-b-D-ribofuranoside \\
\hline AIF & apoptosis-inducing-factor \\
\hline ALL & acute lymphoblastic leukemia \\
\hline AMPK & AMP-activated protein kinase \\
\hline BCRP & breast cancer resistant protein \\
\hline BIR & baculoviral IAP repeat \\
\hline BRAFi & BRAF inhibitors \\
\hline CBP & CREB binding protein \\
\hline CDK4 & cyclin-dependent kinase 4 \\
\hline CDK6 & cyclin-dependent kinase 6 \\
\hline $\mathrm{CPC}$ & chromosomal passenger complex \\
\hline CRPC & castration-resistant prostate cancer \\
\hline $\mathrm{CSCC}$ & cutaneous squamous cell carcinoma \\
\hline CTL & cytotoxic T-lymphocyte \\
\hline CTLA-4 & cytotoxic T lymphocyte-associated antigen 4 \\
\hline DNMT & DNA methyl-transferase \\
\hline EGF & epidermal growth factor \\
\hline EIASD & enzyme-inducing antiseizure drug \\
\hline ER & endoplasmic reticulum \\
\hline FBS & fetal bovine serum \\
\hline FOXO3 & Forkhead box O3 \\
\hline $\mathrm{H} \& \mathrm{E}$ & hematoxylin and eosin \\
\hline HDAC6 & histone deacetylase 6 \\
\hline HGF & hepatocyte growth factor \\
\hline HSP90 & heat shock protein 9 \\
\hline i.p. & intraperitoneally \\
\hline IGF-1 & insulin-like growth factor 1 \\
\hline IGF-1R & insulin-like growth factor 1 receptor \\
\hline INCENP & inner centromere protein \\
\hline JNK & c-Jun N-terminal kinase \\
\hline LRP & lung-resistance-associated protein \\
\hline MEKi & MEK1/MEK2 inhibitor \\
\hline MRP & multidrug resistance-associated protein \\
\hline MTD & maximum tolerable dose \\
\hline mTOR & mammalian target of rapamycin \\
\hline MTPA & microtubule-targeted tubulin-polymerizing agents \\
\hline $\mathrm{NF}-\kappa \mathrm{B}$ & nuclear factors kappa-light-chain-enhancer of activated B cell \\
\hline PD-1 & programmed cell death protein 1 \\
\hline PDGFR $\beta$ & platelet-derived growth factor receptor beta \\
\hline PFS & progression-free survival \\
\hline Pgp & P-glycoprotein \\
\hline PI3K & phosphatidylinositol 3-kinase \\
\hline
\end{tabular}




$\begin{array}{ll}\text { PKA } & \text { protein kinase A } \\ \text { PKC } & \text { protein kinase C } \\ \text { PLK } & \text { polo-like kinase 1 } \\ \text { PTEN } & \text { phosphatase tensin } \\ \text { RLU } & \text { relative luminescence unit } \\ \text { ROS } & \text { reactive oxygen species } \\ \text { RTKs } & \text { receptor tyrosine kinases } \\ \text { SD } & \text { standard derivation } \\ \text { Smac } & \text { second mitochondria-derived activator of caspases } \\ \text { Sp1 } & \text { specificity protein 1 } \\ \text { SSC } & \text { saline-sodium citrate } \\ \text { STAT3 } & \text { signal transducer and activator of transcription-3 } \\ \text { TGI } & \text { tumor growth inhibition } \\ \text { TNF- } \alpha & \text { tumor necrosis factor } \alpha \\ \text { TTP } & \text { time to progression } \\ \text { TUNEL } & \text { terminal deoxynucleotidyltranferase (TdT)-mediated dUTP nick } \\ & \text { end labeling } \\ \text { Vem } & \text { vemurafenib } \\ \text { XAF } & \text { XIAP-associated factor 1 } \\ \text { XIAP } & \text { X-linked inhibitor of apoptosis }\end{array}$




\section{CHAPTER 1. EMERGING DRUG COMBINATION APPROACHES IN MELANOMA THERAPY*}

\subsection{Introduction}

The FDA approvals of ipilimumab targeting the cytotoxic T lymphocyteassociated antigen 4 (CTLA-4), pembrolizumab targeting the programmed cell death protein 1 (PD-1), BRAF inhibitors vemurafenib and dabrafenib, and MEK inhibitor trametinib represent significant milestones in more effective treatment of advanced melanoma. However, it is clear that the use of these single-agent therapies have limitation clinically. For example, ipilimumab only showed $4.5 \%$ objective response rate when used alone in a Phase II clinical trial (1). The efficacy of vemurafenib lasts only 6.7 months before the disease relapses especially in patients with metastatic melanoma (2). Therefore, rational combination approaches are strongly preferred in order to improve the overall patient progression-free survival (PFS), overcome or delay the development of multi-drug resistance and reduce the incidents of side effects (3-6).

In this chapter, we will summarize the emerging combination therapy approaches from both clinical trial and preclinical research in the past five years (7).

\subsection{Combination of Kinase Inhibitors for Melanoma Treatment}

\subsubsection{Combined Inhibitions Targeting Components within the Mitogen-activated Protein Kinase (MAPK) Signaling Pathway}

\subsubsection{Targeting BRAF: mechanism of action, toxicity and drug resistance}

BRAF is a serine/threonine growth signal transduction protein kinase from RAF family which plays important roles in the RAS/RAF/MEK/ERK pathway and directs cell division, proliferation and secretion (8). BRAF inhibitors (BRAFi) are ATP-competitive ligands which inactivate the function of BRAF protein by either stabilizing the inactive form of kinase domain (sorafenib) or preferentially inhibit the active form of the kinase (vemurafenib, dabrafenib) $(9,10)$. Various mutations of $B R A F$ gene have been identified in cancers including melanoma, colorectal and ovarian cancer. Around $60 \%$ of human melanoma adopted the T1799A transversion in exon 15, which lead to BRAF ${ }^{\mathrm{V} 600 \mathrm{E}}$ mutation and the over-activated monomer phosphorylation for $\operatorname{BRAF}^{\mathrm{V} 600 \mathrm{E}}(10,11)$. The

* Reprinted with permission. Wang J, Miller DD and Li W. Emerging Drug Combination Approaches in Melanoma Therapy, Melanoma - Current Clinical Management and Future Therapeutics, Prof. Mandi Murph (Ed.), 2015. ISBN: 978-953-51-2036-0, InTech, DOI: $10.5772 / 59360$. 
two FDA approved BRAFi (vemurafenib and dabrafenib) selectively and potently block the activation of BRAF ${ }^{\mathrm{V} 600 \mathrm{E}}$ and thus inhibit the MAPK signaling pathway.

These drugs show very high clinical efficacy in metastatic melanoma patients harboring the $\mathrm{BRAF}^{\mathrm{V} 600 \mathrm{E}}$ mutation (12-14). Interestingly, in a clinical study which treated 43 patients with any V600 BRAF mutation including the rare V600R variant, five out of the six melanoma patients having V600R mutation had clinical response to the therapy of vemurafenib or dabrafenib (response rate $86 \%$ ) (15).

However, wide type BRAF melanoma tumors do not respond to vemurafenib or dabrafenib inhibition, although they are sensitive to the MEK inhibitors (10).

Paradoxically, in cells with RAS mutation and wild-type BRAF, treatment with vemurafenib or dabrafenib will promote the formation of BRAF-CRAF heterodimer and lead to the activation of subsequent MEK/ERK signaling and cell proliferation (5) as shown in Figure 1-1. This mechanism is used to explain the observation of typical clinical side effects associated with the use of vemurafenib: nearly $25 \%$ of patients developed skin lesions and even cutaneous squamous cell carcinoma (CSCC). In addition, in vitro study has revealed that vemurafenib inhibits multiple off-target kinases including c-Jun N-terminal kinase (JNK), suppresses JNK-dependent apoptosis, and generates CSCC toxicity (16).

\subsubsection{Mechanisms of resistance to BRAF inhibition}

In general, due to alternative pathway activations and inter- and intra-patients melanoma genetic heterogeneity, various mechanisms of resistance to BRAF inhibition have been identified $(11,17-20)$. As we mentioned before, melanoma tumors bearing wide type BRAF are intrinsically resistant to vemurafenib and dabrafenib. Tumor microenvironment also contributes to the innate resistance to BRAF inhibition in melanoma. For example, stromal cells secrete hepatocyte growth factor (HGF), which activates the HGF-receptor MET, MAPK and PI3K-AKT pathways (21).

Eventually, nearly all BRAF mutated melanoma tumors develop acquired drug resistance upon treatment with BRAF inhibitors. The disease progression arises as early as two-month continuous treatment $(19,20)$. The mechanisms of acquired resistance to BRAF inhibition can be generalized into two categories: BRAF $^{\mathrm{V} 600 \mathrm{E}}$-bypass mechanisms and MAPK-bypass mechanisms.

First, the BRAF ${ }^{\mathrm{V} 600 \mathrm{E}}$-bypass mechanisms reactivate MAPK signaling and lead to ERK-dependent tumor cell survival and proliferation (Figure 1-2A). COT, which is coded by gene $M A P 3 K 8$, is a MEK kinase. The overexpression of COT or amplification of $M A P 3 K 8$ directly activates MEK signaling without the participation of RAF protein (22). The mutant of MEK $1^{\mathrm{C} 121 \mathrm{~S}}$ increases catalytic capability and circumvents BRAF to activate basal level of ERK phosphorylation (23). Before the treatment of vemurafenib or dabrafenib, melanoma cells with BRAF ${ }^{\mathrm{V} 600 \mathrm{E}}$ mutation have over-activated monomer BRAF/MEK/ERK cascade which forms an ERK-dependent negative feedback loop. This negative feedback loop reduces the expression of the active RAS-GTP. In the presence of 


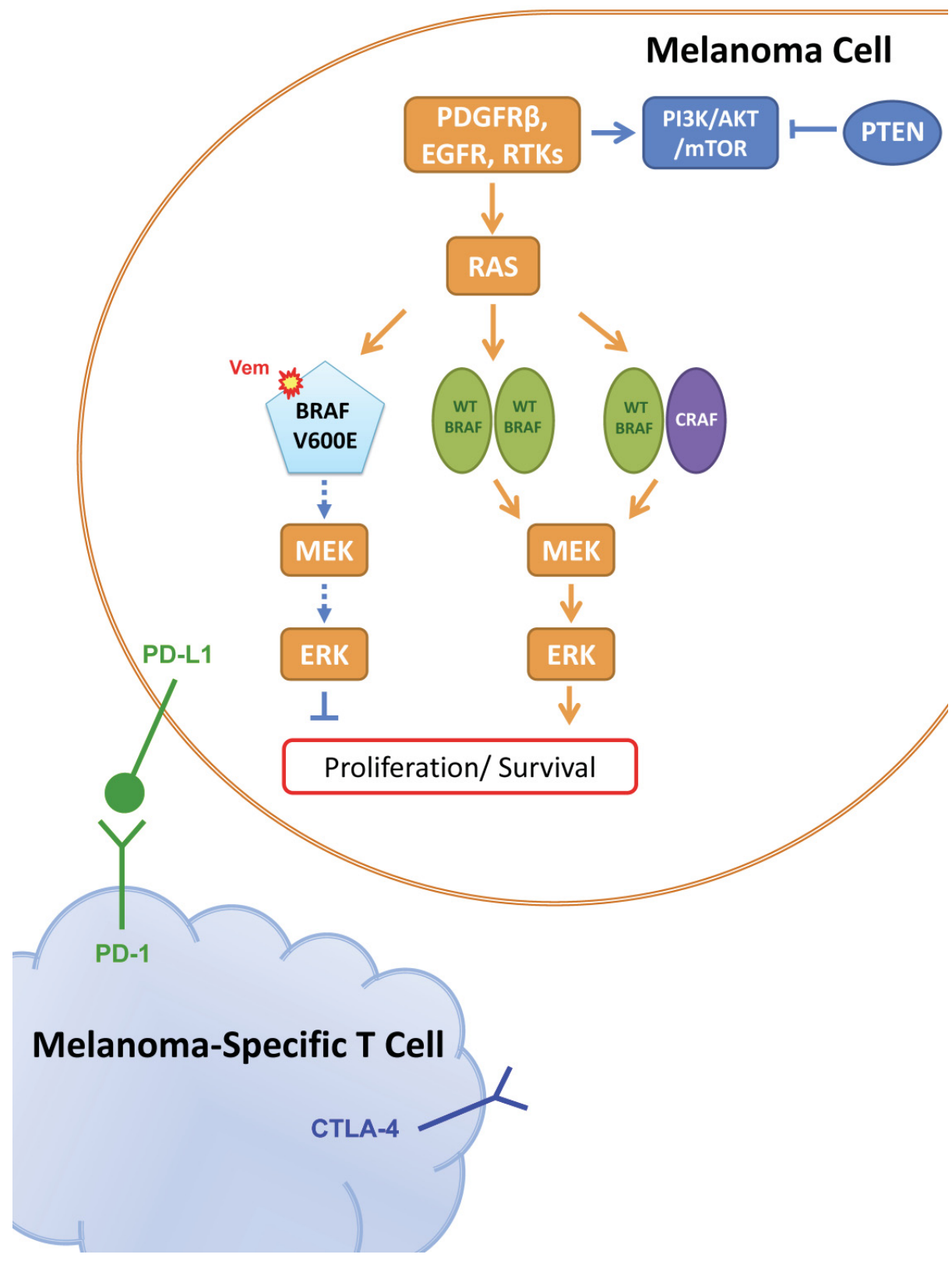

Figure 1-1. The mechanisms of BRAF inhibitor vemurafenib (Vem) action, toxicity and the interaction between melanoma cells with $T$ lymphocytes 


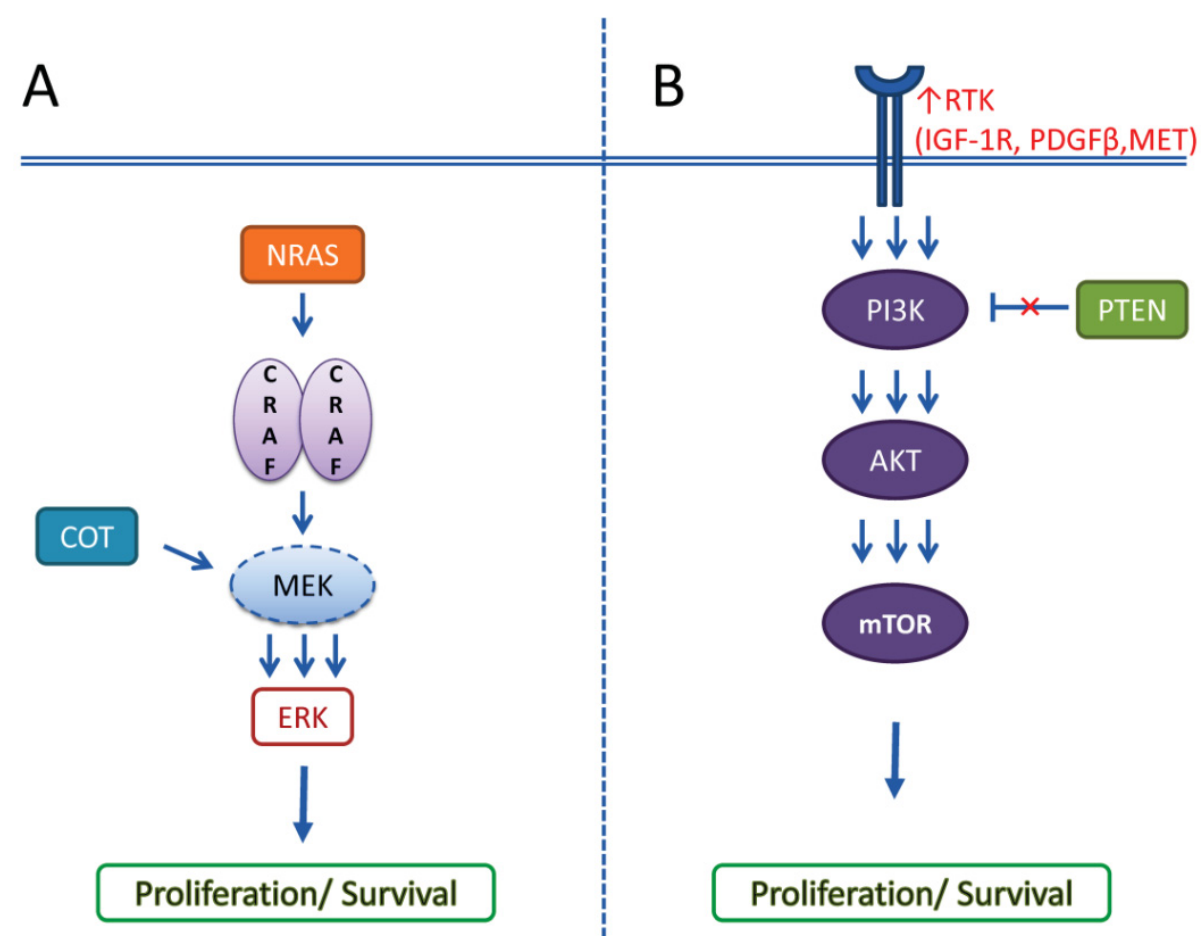

Figure 1-2. The mechanisms of acquired resistance to BRAF inhibition 
vemurafenib or dabrafenib, ERK phosphorylation level is rapidly reduced and the feedback suppression on RAS activation is abolished (Figure 1-1). Therefore, eventually the ERK cascade level is restored through RAS over-activation. NRAS mutants including $\mathrm{NRAS}^{\mathrm{Q} 61 \mathrm{~K}}$ and NRAS ${ }^{\mathrm{Q} 61 \mathrm{R}}$ can drive ERK activation through ARAF or CRAF homo- or hetero-dimers which are alternative MEK activators (24). The combinations of BRAF inhibition plus MEK or ERK inhibition have showed efficacy of overcoming the resistance through these $\mathrm{BRAF}^{\mathrm{V} 600 \mathrm{E}}$-bypass mechanisms $(25-27)$, leading to the recent FDA approval of dabrafenib plus trametinib combination therapy for advanced melanoma.

Second, the MAPK-bypass mechanisms allow melanoma cells to escape from the cytotoxicity of BRAF or MEK inhibition through the activation of ERK-independent survival pathways (Figure 1-2B). The PI3K-AKT signaling pathway can be activated through the overexpression of receptor tyrosine kinases (RTKs), for example, insulin-like growth factor 1 (IGF-1) receptor (IGF-1R) and platelet-derived growth factor receptor beta (PDGFR $\beta$ ) (28). The elevated levels of IGF-1R, PDGFR $\beta$ or HGF can also stimulate another receptor tyrosine kinase, MET, and increase the activity of PI3K. Phosphatase tensin (PTEN) is a negative regulator of PI3K. The PTEN loss-of-function mutation induces the resistance of BRAF inhibition and reduces the PFS of dabrafenib therapy in melanoma patients due to the PI3K activation (29). Moreover, the up-regulation of cyclin D1 can activate cyclin-dependent kinase 4 (CDK4) and 6 (CDK6) and make melanoma cells less dependent on MAPK signaling in cell cycle progressing (30).

Additionally, Jaehyuk Choi et al has reported a BRAF ${ }^{\mathrm{L} 55 \mathrm{H}}$ mutation which changes an amino acid residue in BRAF-vemurafenib interface and causes the resistance to vemurafenib treatment in vitro (31). Since vemurafenib is a substrate of the ATPbinding cassette sub-family $\mathrm{G}$ member 2 (ABCG2), the overexpression of ABCG2 in BRAF $^{\mathrm{V} 600 \mathrm{E}}$ melanoma cell lines has caused the increasing of vemurafenib efflux in vitro (32). The elucidation on the mechanism of acquired-resistance to BRAFi opens a door to rationally design and explore the proper combination strategies to overcome or delay the development of BRAFi resistance.

\subsubsection{Targeting MEK: mechanism of action, toxicity and resistance}

Trametinib, which was approved by FDA in May 2013 as a monotherapy agent against advanced melanoma with $\mathrm{BRAF}^{\mathrm{V} 600 \mathrm{E}}$ and $\mathrm{BRAF}^{\mathrm{V} 600 \mathrm{~K}}$ mutations, is a first-inclass, orally available, allosteric (non-ATP-competitive) MEK1/MEK2 inhibitor (MEKi)(33, 34). It selectively inhibits MEK, the down-stream kinase protein of RAF in the RAS-RAF-MEK-ERK pathway. As a result, melanoma cells with acquired resistance to BRAFi are commonly cross-resistant to MEKi such as trametinib or selumetinib, another selective allosteric MEKi $(25,35)$. This mechanism explains the clinical trial results in which trametinib monotherapy fails to significantly benefit patients who have already developed acquired BRAFi resistance (36). In contrast to the use of a BRAFi, no CSCC side effects are observed among the patients received trametinib treatment in clinical trials $(14,33)$. However, similar to the use of vemurafenib, disease progression occurs within 6-7 months in patients receiving single-agent trametinib treatment (37). 
Nevertheless, a retrospective analysis of 23 patients, who were first treated with MEKi and upon progression with a selective BRAFi, shows that the median time to progression (TTP) has been prolonged to 8.9 months from 4.8 months using a single-agent MEKi or 4.4 months for a single-agent BRAFi treatment, respectively (38). However, a recent clinical trial indicated that if melanoma patients were treated with a BRAFi first then MEKi therapy, no confirmed response was observed (36). This indicates that optimal treatment schedule and sequence is important for the melanoma therapy targeting the MAPK pathway.

\subsubsection{Drug combination targeting MAPK pathway: from lab bench to clinical practice}

Given that the mechanisms of tumor cells develop resistance to BRAFi partially by reactivating the ERK cascade and side effects such as CSCC are RAF-dependent, combining BRAFi with MEKi has attracted lots of research interest in order to further block the MAPK signaling pathway. In vitro and murine models first show the synergistic anti-proliferation and anti-tumor growth effects using the combined BRAFi and MEKi treatment $(10,28,39,40)$. Further, this combination overcomes the acquired resistance to BRAFi $(28,39)$ in both cellular based assay and mouse xenograft models. In addition, the combined inhibition of BRAF-MEK suppresses the paradoxical BRAFiinduced MAPK signal elevation in melanoma cells and reduces the incidences of skin lesions in a rat model (10).

When it comes to the clinical trial data, the combined inhibition of BRAF-MEK has presented significant improvements of major patient benefits (PFS and overall survival). A phase I/II trial (ClinicalTrials.gov, NCT1072175) investigated the combination of oral dabrafenib (150 mg twice per day) plus oral trametinib (1 or $2 \mathrm{mg}$ daily) (combination 150/1 and 150/2) versus monotherapy of dabrafenib (150 mg twice per day) over 108 metastatic melanoma patients bearing either V600E (92 patients) or V600K (16 patients) BRAF mutation $(13,37)$. Median PFS in combination 150/2 group reached 9.4 months, compared to 5.8 months in the dabrafenib monotherapy group (hazard ratio $0.39,95 \%$ confidence interval 0.25 to 0.62 ). The incidence of CSCC adverse events among combination 150/2 group is non-significantly lower than that among monotherapy group $(7 \%$ versus $19 \%, P=0.09)$. But more frequent cases of pyrexia which is not common in trametinib single treatment have been reported in combination $150 / 2$ group (71\%, with recurrent rate $79 \%$ ), as compared with dabrafenib monotherapy group (26\%) (41). These promising data lead to an accelerated FDA approval of the combination of dabrafenib (BRAFi) and trametinib (MEKi) for the treatment of unresectable or metastatic melanoma patients with BRAF V600E or V600K mutation, although further phase III studies with recruitment of more patients comparing the combination therapy with dabrafenib or vemurafenib single treatment (ClinicalTrials.gov, NCT01584648, NCT01597908) are still being assessed.

In addition, several ongoing phase I/II clinical trials now have shown that generally the combination of other BRAFi and MEKi is well tolerated in patients with or without receiving BRAFi treatment before (ClinicalTrials.gov, NCT01271803 
vemurafenib (BRAFi) + cobimetinib (MEKi), NCT01543698 LGX818 (BRAFi) + MEK162 (MEKi)) (42-44) and overall response rate has increased comparing to the monotherapy groups, although the anti-tumor efficacy data haven't been released.

\subsubsection{Combination Targeted Therapy Using Phosphatidylinositol 3-kinase (PI3K)/AKT/Mammalian Target of Rapamycin (mTOR) Inhibitors}

The activation of PI3K/AKT/mTOR pathway have been widely proved to be one of the major mechanisms of intrinsic or acquired resistance to both DNA-methylation agents (e.g. dacarbazine) and targeted BRAF inhibitor therapy (Figure 1-1). Some cell lines that are cross-resistant to both BRAFi and MEKi, are still sensitive to the inhibition of AKT/mTOR(35). On the other hand, mechanistic study revealed evidences of a negative crosstalk between RAF/MEK/ERK and PI3K/AKT/mTOR pathways through RAS kinase. Therefore, when the downstream mTOR function is blocked, PI3K will be able to activate MAPK pathway via a switch of RAS $(45,46)$. These investigations suggest a promising combination strategy of targeting MAPK pathway together with PI3K/AKT/mTOR cascade. Several preclinical studies widely proved that in MAPK inhibition sensitive melanoma cell lines, co-targeting PI3K/AKT/mTOR effectively induces cancer cell apoptosis with down-regulated anti-apoptotic BCL-2 family proteins (35, 47-49). Such a co-targeting strategy can also postpone the emergence of acquired resistance to BRAFi dabrafenib mediated by PTEN mutation or disruption $(50,51)$. Further, the dual inhibition of two pathways has successfully overcome NRAS mutation mediated resistance to MAPK blockade in vitro and induced xenograft tumor regression in vivo $(35,39,52)$. Finally, the combination of vemurafenib (BRAFi) or selumetinib (MEKi) with BEZ235 (dual PI3K and mTORc1/2 inhibitor) has been shown to overcome the PDGFR $\beta$-driven resistance to MAPK pathway inhibition (53).

A series of Phase I studies have evaluated the clinical relevance of the combination therapy which co- targets PI3K/AKT/mTOR and RAF/MEK/ERK pathways in terms of the incidence on severe side effect and anti-tumor efficacy in 236 patients. These patients have advanced cancers including melanoma, colorectal, pancreatic and non-small cell lung cancers. Results from three combination groups (AKTi MK2206 + MEKi selumetinib, NCT01021748; AKTi GSK2141795 + MEKi trametinib, NCT01138085; mTOR inhibitor everolimus + MEKi trametinib, NCT 00955773) are compared to the single treatment groups (54). Overall, the combination therapy did not provide significant increase of tumor control rate (64.6\% for combination, $52.7 \%$ for monotherapy, $P=0.16$ ), although all five colorectal patients with co-activation of both pathways in combination group achieved tumor regression to varied extent between $2 \%$ and $64 \%$. However, this combination strategy causes significant higher rates of drugrelated grade III and above side effects $(53.9 \%$ for combination, $18.1 \%$ for monotherapy, $P<0.001)$. Furthermore, two clinical trials which involve the combination therapy of BRAFi or MEKi with AKTi DNE3 recently have been terminated due to the safety concerns of the toxic properties of DNE3 (ClinicalTrials.gov, NCT02087254 and NCT02095652). Nevertheless, in another ongoing phase I/II trial which measures the safety and efficacy of a well-tolerated pan-PI3K inhibitor BKM120 combined with 
vemurafenib therapy, preliminary data reveals that a vemurafenib-refractory melanoma patient with PTEN expression achieved a 35.9\% reduction in target tumor (ClinicalTrials.gov, NCT01512251) (55). In general, drug-related toxicity is one of the major issues for this cross-pathway targeted combination therapy and patients genetic profiling is very important to achieve the maximum objective response.

\subsubsection{Combining Targeted Therapy with Anti-angiogenic Agents}

Melanoma is a vascular tumor. The abnormal expression of the epidermal growth factor (EGF) family protein and the up-regulation of EGFR-mediated alternative survival pathway have critically shaped the response of melanoma to the current chemotherapy agents (56-59). In a recent study by Sun et al, six out of sixteen melanoma cell lines display acquired EGFR expression after the development of resistance to BRAFi and MEKi (60). Even before the FDA approval of BRAFi and MEKi, the combination of bevacizumab, a recombinant human monoclonal antibody VEGF inhibitor, with a specific chemotherapy agent (for example, fluorouracil (61) or fotemustine (62)), has become a first-line treatment for metastatic melanoma patients. Clinical trials that study the combination of anti-angiogenic agents with cytotoxic agents have achieved promising anti-tumor activity, although tolerability issues exist (63). VEGF blockage has been shown to enhance the efficacy of a GM-CSF-secreting immunotherapy in vitro (64). In addition, a VEGF receptor-2 inhibitor, semaxanib, prolonged both the complete and partial response time of an immunomodulatory drug, thalidomide, over 10 recurrent metastasis melanoma patients without showing significant drug-drug interaction toxicity in a phase II trial (65).

Along with the rapid development of targeted melanoma therapeutics, the combined inhibition of VEGFR plus PDGFR or mTOR has shown synergy anti-tumor effects on mouse models of B16 metastatic melanoma without increasing toxicity (66, 67). A large-scale, unbiased drug screening study, which aims to discover effective genotype selective combinatorial therapeutics of vemurafenib-resistant BRAF and RAS mutant melanoma, identifies a triple BRAF+EGFR+AKT inhibition as highly effective approach (3). In the year of 2010, combination of bevacizumab with an mTOR inhibitor, everolimus, was evaluated in a phase II trial for patients with metastatic melanoma (68).

The treatment was well tolerated in most patients. Seven out of fifty-seven patients $(12 \%)$ receiving combination therapy have shown major responses, although the median PFS was only 4 months. This year (2014), in a phase II trial that combines bevacizumab and sorafenib, which is an inhibitor of both RAF kinase and VEGFR-2/PDGFR- $\beta$ signaling, no objective tumor responses are seen in all the fourteen patients receiving treatment $(69$, 70). Interestingly, the median TTP of patients with low VEGF $(<300 \mathrm{pg} / \mathrm{ml})$ was longer than that of patients with high VEGF (50 weeks versus 15 weeks, $P=0.02$ ). Therefore, the levels of VEGF in patients do influence the tumor progression profile (ClinicalTrials.gov, NCT00387751). 


\subsubsection{Combination Therapy Using Targeted Therapy with Versatile Chemotherapy Agents}

Since the abnormally activated (phosphorylation) of ERK and AKT constitutively exist in melanoma cells and promote the disease progression especially metastasis, blocking ERK or AKT pathway can sensitize the metastatic melanoma to the apoptosis induced by chemotherapeutic agents including cisplatin, temozolomide, DTIC and arsenite (71-73). With the understanding of tumor biology about the programmed cell apoptosis and the rapid development of agents that can trigger the cell death process in melanoma, the combination of a MAPK inhibitor with a BCL-2 inhibitor (ABT-737 (74) or navitoclax (75)), or a MDM2 antagonist nutlin-3 (76), has synergistically induced apoptosis of melanoma in vitro and suppressed xenograft tumor growth in vivo. A comparative analysis on the samples collected from patients receiving vemurafenib or dabrafenib/trametinib combination treatment showed that BCL-2 expression level is closely related to the onset of MAPK inhibition resistance (75). Clinical trials are being conducted to investigate the combination of BCL-2 inhibitor (BH3 mimetics) navitoclax and vemurafenib (75).

Due to the heterogenetic characteristics of melanoma disease, Vultur A et al (77) recently report that MEK or BRAF inhibition can potentially strengthen the invasion property of human melanoma cells by about $20 \%$. As a result, co-inhibiting kinases that are actively involved in cell invasion process, such as RTK, STAT3 and Src, together with MEK inhibition has effectively abolished the invasive phenotype and further caused the tumor cell death in a 3D matrix model.

Metformin, a biguanide oral anti-diabetic drug, has been discovered with antitumor activity in various cancer types including melanoma. Although the exact mechanisms remain to be elucidated, accumulating data suggest that metformin can activate AMP-activated protein kinase (AMPK) and thus increase the activities of VEGF and ERK in $\mathrm{BRAF}^{\mathrm{V} 600 \mathrm{E}}$ mutated melanoma cells (78). AMPK negatively regulates malignant cell proliferation and viability (79). The combination of vemurafenib and metformin has shown synergistic anti-proliferative effects on six out of eleven tested $\mathrm{BRAF}^{\mathrm{V} 600 \mathrm{E}}$ melanoma cell lines (80). Pilot clinical studies that evaluate the safety and efficacy of metformin combination therapies (plus dabrafenib or trametinib) are now recruiting patients (ClinicalTrials.gov, NCT0184000, NCT02143050).

Unlike the cutaneous melanoma, over-activation of MAPK pathway in uveal melanoma is associated GNAQ or GNA11 mutations instead of BRAF or RAS mutations (81). Protein kinase C (PKC) inhibitors such as enzastaurin or AEB071 induce apoptosis in GNAQ-mutant but not in GNAQ wild type uveal melanoma cells (82). The level of ERK phosphorylation also decreases in these cells when they are treated using PKC inhibitors (82). Chen et al. has recently confirmed the synergy of the combination using a PKC inhibitor with a MEKi (PD0325901 or MEK162) in GNAQ/11 mutant uveal melanoma cells $(83)$. 
Understanding the mechanisms of resistance to MAPK inhibition in melanoma can lead to rational combination designs in order to overcome acquired drug resistance to BRAF inhibitors. For example, our lab recently identified a synergistic combination in which a novel tubulin inhibitor ABI-274 combined with vemurafenib could overcome the acquired vemurafenib-resistance (84). This combination treatment effectively arrested the vemurafenib-resistant melanoma cells in both $\mathrm{G}_{0} / \mathrm{G}_{1}$ and $\mathrm{G}_{2} / \mathrm{M}$ phases and induced strong apoptosis through the down-regulation of AKT phosphorylation. In addition, the combination of a MEKi (TAK-733) with an Hsp90 inhibitor (ganetespib) induces tumor regressions in vemurafenib-resistant xenograft models also through the depletion of AKT signaling (85). With the finding that up-regulated cyclin D1 expression is critical for the survival of vemurafenib-resistant cells, a selective inhibitor of cyclin dependent kinase (CDK) 4/6, LY2835219, has been reported to overcome the reactivation of MAPK signaling in vemurafenib-resistant $\mathrm{BRAF}^{\mathrm{V} 600 \mathrm{E}}$ melanoma (86).

\subsection{Combinations Involving Immunotherapy in Melanoma Treatment}

\subsubsection{Combined Blockade of Immuno-checkpoints}

Given the unsatisfactory results of cytokine-based melanoma immunotherapy (recombinant interferon- $\alpha 2 \mathrm{~b}$ and high dose interleukin-2) in the past decade, the development and approval of ipilimumab (anti-cytotoxic T lymphocyte-associated antigen 4 (CTLA-4) monoclonal antibody) in 2013 have marked a breakthrough of immune-checkpoints blockade therapy (87). CTLA-4 (CD152) expresses on the surface of active T-lymphocytes and inhibits the initial T-cell proliferation and migration to the tumor tissue (88). CTLA-4 antibodies preferentially target the suppressive regulatory $\mathrm{T}$ cells and prevent them from being hijacked by tumors (89). In a double-blinded phase III study in 676 patients with pretreated and refractory metastatic melanoma, ipilimumab at the dose of $3 \mathrm{mg} / \mathrm{kg}$ achieved a median OS of 10 months (87). In a meta-Kaplan-Meieranalysis of data collected from 1,861 melanoma patients in a clinical trial, a plateau of survival curve starts from around 3 years after ipilimumab treatment with follow-up extends as long as ten years, indicating a long-term survival benefits of ipilimumab therapy (ClinicalTrials.gov, NCT01844505). In addition, ipilimumab showed good tolerance and efficacy in several other clinical trials in which it was combined with a standard chemotherapy agent such as dacarbazine, fotemustine or temozolomide (90).

Another success of immune-check point blockade strategy is the development of anti-programmed death-1 (PD-1) antibodies, represented by pembrolizumab (MK-3475) and nivolumab (91, 92). Pembrolizumab, as the first-in-class PD-1 inhibitor, has obtained FDA approval in September 2014 for patients with advanced or unresectable melanoma. The cDNA of PD-1 (CD279) is first cloned in programmed death T cells although PD-1 itself does not directly induce apoptosis. PD-1 is over-expressed on the surface of dysfunctional activated T-cells and contributes to the maintenance of T cell dysfunction (exhaust) phenotype and proliferation disability in the tumor site (93). Two counter receptors of PD-1 have been identified: PD-L1 and PD-L2. PD-L1 is more frequently and 
exclusively expressed in various tumor cells; therefore, antibodies targeting PD-L1 (MPDL3280A and BMS-936559) also have anti-tumor activity in advanced cancer including melanoma $(92,94)$. The PD-1-PD-L1 ligation retards the recognition and destroying of tumor cells by CD8+ cytotoxic T-lymphocytes (88). As a result, blocking PD-1 or PD-L1 will reverse the cancer cell immune escape. Because both CTLA-4 and PD-1 are key negative receptors that cooperatively modulate the adaptive immune response in tumor progression, their combination has been shown to be synergistic in B16 melanoma tumors without overt toxicity (95).

In a cohort phase I trial that studied the concurrent administration of ipilimumab and nivolumab to 53 patients with advanced, treatment-resistant melanoma, more than $80 \%$ tumor reduction was observed in $30 \%$ patients after 12 weeks treatment at the maximum tolerated dose. Twenty-one out of fifty-three patients had objective responses and over $80 \%$ of these patients had tumor regression. Grade 3/4 adverse events are diagnosed in 53\% patients but the toxicities are manageable with immune-suppressants (96). Consequential trials with more enrollment number of patients are necessary to further evaluate the safety and efficacy of this promising double immune-checkpoints blockage therapy comparing with each of its monotherapy regiments.

Finally, combinatorial clinical trials using ipilimumab with other immunotherapy agents have shown some favorable therapeutic benefits. For example, combination of ipilimumab with peginterferon $\alpha-2 b$ (pegylated interferon $\alpha-2 b$ ) in patients with unresectable melanoma both demonstrated significant increase of response rate and OS comparing with the monotherapy arm $(97,98)$ in recent phase I trials.

\subsubsection{Combined Therapy Inhibiting both Immuno-checkpoint and MAPK Signaling Pathway}

Checkpoint blockade immunotherapy and MAPK targeted chemotherapy have distinct clinical profiles. For example, targeted therapy has relative higher initial response rate $(\sim 60 \%$ for BRAFi) with rapid onset of effect, but its efficacy restrictively rely on the continuous treatment and the therapeutic response is usually not durable due to the quick development of acquired drug resistance. In contrast, immunotherapy has much a lower response rate ( $4.5 \%$ for ipilimumab), delayed onset of effect and difficulty in predicting patient outcome, but it has shown potentially durable responses and long-term survival benefit even off treatment. In addition, since the MAPK pathway is not required in the process of anti-tumor immune response, blocking MAPK signaling should not interfere with the efficacy of checkpoint blockade immunotherapy. Therefore, it seems very rational that the combination of a MAPKi and an immunotherapy agent such as ipilimumab or pembrolizumab can maximize the therapeutic benefits in advance melanoma.

Interestingly, BRAF and MEK inhibition displayed an "endogenous vaccine-like" effects in melanoma cells (99). Cytotoxic agents like BRAFi induce tumor cell death and promote the uptake and presentation of tumor antigens to the effector immune cells ( $\mathrm{T}$ 
cells and B cells) through antigen-presenting cells (55). MEK inhibition, BRAF ${ }^{\mathrm{V} 600 \mathrm{E}}$ RNA silencing or BRAF inhibition by PLX4720 increases the CD4+ and CD8+ lymphocytes mediated T-cell infiltration and reduce the level of immune-suppressants including IL-6, IL-10 or VEGF (100-102) in mice. The expression of PD-L1 is found to be elevated in BRAFi-resistant melanoma cells and it is mediated through the off-target activity of BRAFi in JUN and STAT3 signaling (103). However, Vella et al has published a paper in 2014 and stated that they have not found any impact of dabrafenib treatment on T lymphocytes. trametinib alone or in combination with dabrafenib has suppressed $\mathrm{T}$ lymphocyte proliferation, cytokine secretion and antigen-specific expansion in their isolated T lymphocyte and monocyte-derived dendritic cells. These findings should be carefully tested in vivo to evaluate the clinical relevance (104).

As for the clinical practice, dose-limiting hepatotoxicity issues have led to the premature termination of the first phase I study on combination of ipilimumab with vemurafenib (ClinicalTrials.gov, NCT01400451). This signified the complexity of adverse effect in combined therapy of immune-regulating agents and kinase inhibitors. Another phase I study of ipilimumab plus dabrafenib, or ipilimumab plus the combination of dabrafenib with trametinib is still active and a phase II study is exploring the safety and efficacy of sequential administration of vemurafenib followed by ipilimumab (ClinicalTrials.gov, NCT01767454, NCT01673854). The data of these most recent trials will be released in the near future.

\subsection{Conclusion}

Extensive efforts and remarkable progresses have been made to discover and investigate rational approaches in combination melanoma therapy since the recent approval of MAPKi and immune checkpoints blockade antibodies. A number of new targeted or immune drugs for metastatic melanoma are currently under commercial development or late stage clinical trials, some of which will likely be approved in the next few years. Quality of life for many melanoma patients has been dramatically increased. However, significant challenges still remain. While some clinical evidence has really raised the expectation of survivals for patients with advanced melanoma, the benefits of combination therapy are usually accompanied by limitations. Comprehensive genetic profile and tailored patient matching is essential for targeted therapy, while biomarkers are critical to predict the patient immunotherapy response. Drug-related toxicity for combination treatment usually is not a simple one-plus-one situation, and potential drug-drug interactions, especially the combination of a targeted agent with an immunotherapeutic agent must be carefully evaluated in order to achieve both fast and durable responses. Adverse effects should be closely monitored and potential alternative dosing regiments is worth further exploration. Optimized dose schedule may help to delay the resistance development and reduce the frequency of adverse effect. For example, intermittent doses of BRAFi was able to enhance the tolerance in combination with immunotherapy, decrease the paradoxical MAPK activation, which might be the main cause of severe toxicity in clinical trial (105). Solid evidence of synergistic combination in preclinical research must be established before clinical trial conduction. 
In fact, with the relatively large number of available targeted agents and immunotherapeutic agents for metastatic melanoma, the huge number of possible drug combinations coupled with dosing sequences or schedules already presents a significant challenge in designing proper clinical trials. To test all the possible drug combinations along with different dosing sequences clinically will not only have low benefits to patients, but is also a huge financial burden to the society. Carefully designed, predictive preclinical studies will be essential to provide critical supports for rational prioritization of clinical trials using drug combinations. Finally, clear understandings of various combination mechanisms and patient genetic profiles are critically important for the development of new combination approaches, prediction of expected therapy response and potential side effects. With the rapid advances in this field, it is likely that optimal combination treatments will greatly improve the management of advanced melanoma in cancer patients. 


\section{CHAPTER 2. OVERCOMING ACQUIRED RESISTANCE TO BRAF INHIBITORS BY NOVEL SYNERGISTIC DRUG COMBINATION*}

\subsection{Introduction}

The sustained clinical activity of vemurafenib in melanoma patients with $\mathrm{BRAF}^{\mathrm{V} 600 \mathrm{E}}$ mutation is limited by the rapid development of acquired resistance (106108). Several mechanisms have been proposed in the literature $(28,109-111)$, including the amplification of the BRAF oncogene (112), the up-regulation of CRAF expression (113), oncogenic activation of NRAS (114), up-regulated EGFR-SFK-STAT3 pathway (115), gatekeeper mutations (116-118), up-regulation of growth factor receptors such as insulin-like growth factor 1 receptor (IGF1R) (46) or platelet-derived growth factor receptor (PDGFR) (114), and other resistance mechanisms (21, 119). Several methods to maintain phosphorylated extracellular-signal-related kinase 1 and 2 (p-ERK1/2) levels in the presence of BRAF inhibitor drugs have been described, including ERK-kinase 1 (MEK1) mutation, recruitment of alternative MEK1/2 activators, RAS mutation or upregulation of receptor tyrosine kinases (RTKs). Thus in many cases, vemurafenibresistant cells are cross-resistant to MEK inhibitors $(35,109,120)$.

Drug combinations using agents with distinct anti-cancer mechanisms can enhance tumor response and patient survival, especially in the treatment of advanced cancer patients (121-123). Combinations of vemurafenib with agents targeting the same mitogen-activated protein kinase (MAPK) pathway such as MEK or ERK inhibitors show clinical efficacy $(28,37,39,124)$, however they can only arrest cells in the $\mathrm{G}_{0} / \mathrm{G}_{1}$ phase. Such combination strategies are unlikely to be effective against resistant cells that can escape from this cell cycle arrest. Our preliminary study showed that the chronically selected vemurafenib-resistant human melanoma cells could not be blocked in the $\mathrm{G}_{0} / \mathrm{G}_{1}$ phase by vemurafenib at the effective concentration to sensitive parental cell line, and the vemurafenib-resistant cells readily progressed into the $\mathrm{G}_{2} / \mathrm{M}$ phase. Thus, we hypothesized that a combination of vemurafenib with a compound that strongly induces the subsequent $\mathrm{G}_{2} / \mathrm{M}$ phase block should capture vemurafenib-resistant cells leaking from $\mathrm{G}_{0} / \mathrm{G}_{1}$ arrest, and thus produce a strong synergy.

We recently discovered a novel class of anti-mitotic agents, represented by the 2aryl-4-benzoyl-imidazoles (ABIs) scaffold (125-128). ABI-231 and ABI-274 are among our most potent $\mathrm{ABI}$ compounds discovered to date with anti-proliferation $\mathrm{IC}_{50}$ values in the low nanomolar (nM) range in several melanoma cell lines. They bind to tubulin at the colchicine binding site (129).

*Modified with permission. Wang J, Chen J, Miller DD, Li W. Synergistic combination of novel tubulin inhibitor ABI-274 and vemurafenib overcome vemurafenib acquired resistance in $\mathrm{BRAF}^{\mathrm{V} 600 \mathrm{E}}$ melanoma. Mol Cancer Ther. 2014;13: 16-26. 
Compared with many existing tubulin inhibitors such as paclitaxel and vinblastine, ABIs can effectively circumvent several clinically relevant multidrug resistant mechanisms, including drug resistance mediated by P-glycoprotein (Pgp), multidrug resistance-associated proteins (MRPs), and breast cancer resistant proteins (BCRP). An in vivo study indicated that ABIs significantly inhibited melanoma lung metastasis in mice (129). In the current study, we tested our hypothesis of synergistic cell cycle arrest by the combinations of vemurafenib with ABIs or docetaxel in a panel of $\mathrm{BRAF}^{\mathrm{V} 600 \mathrm{E}}$ mutant parental melanoma cell lines and chronically selected vemurafenibresistant A375RF21 and MDA-MB-435 VemR sublines (28). The established vemurafenib-resistant A375RF21 cells were used in vitro and in vivo as the disease relapse model to test whether our proposed synergistic drug combination would be of potential therapy benefit in associated clinical vemurafenib resistance.

\subsection{Materials and Methods}

\subsubsection{Reagents and Cell Lines}

Vemurafenib, selumetinib, trametinib, sunitinib (malate salt) and docetaxel were purchased from LC Laboratories (Woburn, MA). ABI-274 was synthesized as described (126). Human melanoma A375 cell line was acquired from ATCC (Manassas, VA). Human melanoma WM164 and MDA-MB-435 cells were obtained from Dr. Meenhard Herlyn (Wistar Institute, Philadelphia, PA), and Dr. Robert Clarke (Georgetown University, Washington, DC), respectively. MEK inhibition resistant 451LuMR and BRAF inhibition resistant WM983B BR cells were gifts from Dr. Meenhard Herlyn (Wistar Institute, Philadelphia, PA). All cell lines were authenticated prior to use for this study. A375, WM164 and MDA-MB-435 cells were cultured in DMEM medium (Mediatech, Inc., Manassas, VA), supplemented by 10\% fetal bovine serum (FBS, Atlanta Biologicals, Lawrenceville, GA), 1\% antibiotic/antimycotic mixture and $5 \mu \mathrm{g} / \mathrm{mL}$ bovine insulin (Sigma-Aldrich, St. Louis, MO). 451LuMR and WM983B BR cells were maintained in 5\% FBS supplemented DMEM medium with $1 \mu \mathrm{M}$ trametinib or vemurafenib, respectively.

Vemurafenib-resistant melanoma cells were chronically selected by culturing A375 or MDA-MB-435 cells in increasing concentrations of vemurafenib following the reported method (28) for at least three months. A375RF21 and MDA-MB-435 VemR cells are maintained in full growth medium containing $2.5 \mu \mathrm{M}$ vemurafenib.

\subsubsection{Cell Proliferation and In Vitro Combination Assay}

Cell proliferation was investigated using the MTS or SRB assay as described previously $(125,126,129)$. An in vitro study of the combination of vemurafenib and the tubulin inhibitors was designed and conducted using CalcuSyn software (Biosoft, Ferguson, MO) with five duplicates of each treatment set. Drug concentrations were 
selected based on the $\mathrm{IC}_{50}$ value of each drug tested from a pilot study. Synergism, additive activity or antagonism was determined through the Chou-Talalay method (130), showing a combination index (CI) as calculated in the software output.

\subsubsection{Cell Cycle Analysis}

Flow cytometry analysis was performed as described before (129). To determine cell cycle distributions in the $\mathrm{G}_{2}$ and $\mathrm{M}$ phases, cells were harvested with trypsin, stained using anti-phospho-histone H3 - AlexaFluor ${ }^{\circledR} 488$ antibody on ice for one hour in the dark, followed by stained using PI/RNase solution for 30 minutes at room temperature in the dark per the manufacturer's instructions (\#FCCH025103, EMD Millipore Corporation, Ballerica, MA). Data were further processed and graphs were prepared using the Modfit 2.0 program (Verity Software House, Topsham, ME).

\subsubsection{Tubulin Polymerization Assay}

HTS-tubulin polymerization assay was performed as described previously (127) using a commercial kit following the manufacturer's instructions (\#BK004P, Cytoskeleton, Inc., Denver, CO). The absorbance at $340 \mathrm{~nm}$ was kinetically recorded every $1 \mathrm{~min}$ for $45 \mathrm{~min}$ at $37^{\circ} \mathrm{C}$ by the SYNERGY HT micro-plate reader (Bio-Tek Instruments, Winooski, VT).

\subsubsection{Western Blot Analysis}

At the indicated time treatment, A375RF21, MDA-MB-435 VemR, A375, MDAMB-435 or WM164 cells were collected to investigate levels of relevant cascade protein or apoptosis markers by western blots. The following primary rabbit antibodies (Cell Signaling Technology, Inc., Danvers, MA) were used: anti-phospho-ERK1/2 (Thr202/Tyr204; \#9101), anti-p44/42 MAPRK (ERK1/2; \#9102), anti-phospho-AKT (Ser473; \#9271), anti-AKT (\#9272), anti-cyclin D1 (\#2978); anti-cleaved PARP (\#9185), anti-cleaved caspase-3 (\#9664), anti-RAS (\#3339), anti-ARAF (\#4432), anti-BRAF (\#9433), anti-CRAF (\#9422), phosphor-PI3 kinase p85 (Tyr458)/ p55 (Tyr199) (\#4228), anti-PTEN (\#9188), anti-PDGF receptor $\beta$ (\#3169), or anti-GAPDH (\#3683). Target proteins were detected by incubating with $1 \times$ LumiGLO $^{\circledR}$ reagent (Cell Signaling, \#7003) for one minute and exposed to x-ray film. The films were scanned with grey scale and lane intensities were quantified with the ImageJ software (NIH, Bethesda, MD, USA).

\subsubsection{Apoptosis Detection}

A375RF21 cells were seeded in 6-well plates $\left(1 \times 10^{6}\right.$ per well $)$ and treated with growth medium containing $0.5 \%$ DMSO, vemurafenib, ABI-274, docetaxel or the indicated combinations. After 48 hours of incubation, apoptosis analysis was performed 
using the Annexin V-FITC Apoptosis Detection Kit (Abcam, Cambridge, MA) as per manufacturer's instructions and analyzed by a BD LSR-II cytometer (BD Biosciences, San Jose, CA).

\subsubsection{Vemurafenib-resistant Tumor Xenograft and Treatment}

Seven to eight week old male nude mice were purchased from Charles River Laboratories International, Inc. (Wilmington, MA). A375RF21 cells were suspended in ice-cold phenol red-free and FBS-free DMEM medium and mixed with high concentration Matrigel (BD Biosciences, San Jose, CA) at a ratio of 1:1 right before use. $100 \mu \mathrm{L}$ of this mixture containing $3 \times 10^{6}$ cells was injected subcutaneously (s.c.) to the left-side dorsal flank of each mouse. One week after the inoculation, the mice were randomized into four groups ( $n=7$ for the initial low dose and $n=5$ for subsequent high dose drug combination) and the treatments started. ABI-274 or vemurafenib was diluted in PEG300 (Sigma-Aldrich, St. Louis, MO) and administered through intraperitoneal (i.p.) injection once per day, 5 days per week for three continuous weeks. Vehicle control group was i.p. injected with same volume $(100 \mu \mathrm{L})$ of PEG300 at the same dosing frequency. At the end of the experiments, mice were euthanized and tumor tissues were isolated, weighted and then fixed in $10 \%$ buffered formalin phosphate solution. Tumor volume and body weight of each mouse were evaluated three times a week.

We calculated the tumor volume using formula $a \times b^{2} \times 0.5$, where $a$ and $b$ represented the larger and smaller tumor diameters (129). Data was showed as mean \pm $\mathrm{SD}$ for each group and plotted as a function of time. Tumor growth inhibition (TGI) was calculated as $100-100 \times\left[\left(T-T_{0}\right) /\left(C-C_{0}\right)\right]$, and tumor regression was calculated as $(T-$ $\left.T_{0}\right) / T_{0} \times 100$, where $T, T_{0}, C$ and $C_{0}$ are the mean tumor volume for the specific group on the last day of treatment, mean tumor volume of the same group on the first day of treatment, mean tumor volume for the vehicle control group on the last day of treatment and mean tumor volume for the vehicle control group on the first day of treatment, respectively $(28,107)$.

\subsubsection{Pathology and Immunohistochemistry (IHC) Analysis}

Tumor tissues fixed in formalin buffer for over one week were stained with hematoxylin and eosin. For IHC analysis, the following primary antibodies were used: rabbit anti-Ki67, anti-phospho-AKT (Ser473), and anti-phospho-ERK1/2 (Thr202/Tyr204) (\#9027; \#4060; \#4376, Cell Signaling Technology). Anti-S100 primary antibody was purchased from Abcam (\#ab868, Abcam Inc., Cambridge, MA). Analyses were performed following manufacturer's protocols. 


\subsubsection{Statistical Analysis}

Data were analyzed using Prism Software 5.0 (GraphPad Software, Inc., San Diego, CA). The statistical significance $(P<0.05)$ was evaluated by Mann-Whitney Rank Test, nonparametric $t$-test and one-way ANOVA for in vitro apoptosis detection and xenograft study. Treated groups were compared with the vehicle group and combination treatment groups were compared with the groups that received single agent treatment, accordingly.

\subsection{Results}

\subsubsection{Combination of Vemurafenib with ABI Compound Showed Strong Synergies in Parental BRAF ${ }^{\text {V600E }}$ Mutant Melanoma Cell Lines}

We started by testing ABI compounds (ABI-231 and ABI-274) to evaluate their combination effects with vemurafenib on parental human A375, MDA-MB-435 and WM164 cells which were all BRAF ${ }^{\mathrm{V} 600 \mathrm{E}}$ mutant cell lines. Two well-known tubulin inhibitors, docetaxel and colchicine, were included for comparison (Table 2-1). We found that calculated $\mathrm{CI}$ values for combinations of ABI-231 or ABI-274 and vemurafenib was as low as 0.22 (in A375 cell line) and 0.10 (in MDA-MB-435 cell line) at $\mathrm{ED}_{50}$, showing the combination treatment had strong synergistic effects in vitro.

\subsubsection{Combination of ABI-231 and Vemurafenib Produced Synergistic Cell Cycle Arrests in Vemurafenib-sensitive A375 Cells}

ABI compounds as novel tubulin inhibitors arrest cancer cells including human melanoma $\mathrm{A} 375$ at $\mathrm{G}_{2} / \mathrm{M}$ phase, but vemurafenib as a BRAF inhibitor blocks cancer cell progressing from $\mathrm{G}_{1}$ phase. As shown in Figure 2-1, cell cycle distribution quantitative data revealed that single treatment of $\mathrm{ABI}-231$ at $20 \mathrm{nM}$ generated $43 \pm 8 \%$ or $70 \pm 2 \%$ of $\mathrm{G}_{2} / \mathrm{M}$ arresting after $12 \mathrm{~h}$ or $24 \mathrm{~h}$ incubation. And $60 \pm 4 \%$ or $91 \pm 1 \%$ cells were accumulated in $\mathrm{G}_{1}$ phase when they were treated by vemurafenib at $2 \mu \mathrm{M}$ for $12 \mathrm{~h}$ or 24 h. The combination of compound ABI-231 with vemurafenib displayed interesting "double" cycle cell arresting pattern at both $\mathrm{G}_{1}$ and $\mathrm{G}_{2} / \mathrm{M}$ phases in $\mathrm{A} 375$ cells from $12 \mathrm{~h}$ incubation, which contributed to the combined synergistic anti-proliferation effects.

\subsubsection{Combination of ABI-231 and Vemurafenib Affected Related Protein Levels in Vemurafenib-sensitive A375 Cells}

To preliminarily investigate the mechanism of synergy combination, we performed western blotting to check the levels of related proteins in A375 cells after either single or combined treatment for $24 \mathrm{~h}$, comparing with the DMSO vehicle control group. Representative results are shown in Figure 2-2. Since A375 is a vemurafenib- 
Table 2-1. Combination of vemurafenib with tubulin inhibitors showed synergistic effect in the parental $\mathrm{BRAF}^{\mathrm{V} 600 \mathrm{E}}$ melanoma cell lines as indicated by $\mathrm{CI}$ values determined at $\mathbf{E D}_{50}$

\begin{tabular}{lccc}
\hline $\begin{array}{l}\text { Treatment } \\
\text { +vemurafenib }\end{array}$ & A375 & MDA-MB-435 & WM164 \\
\hline ABI-231 & 0.22 & 0.23 & 0.66 \\
ABI-274 & 0.32 & 0.10 & 0.61 \\
Docetaxel & 0.50 & 0.55 & 0.54 \\
Colchicine & 0.47 & 0.78 & 1.28 \\
\hline
\end{tabular}

The combination index $(\mathrm{CI})$ values at $\mathrm{ED}_{50}$ were calculated based on the results from cell viability MTS assay $(n=5)$. CI $<0.9$ indicates synergism; $0.9 \leq \mathrm{CI} \leq 1.1$ indicates additive effect; $\mathrm{CI}>1.1$ indicates antagonism between the two tested drugs. 

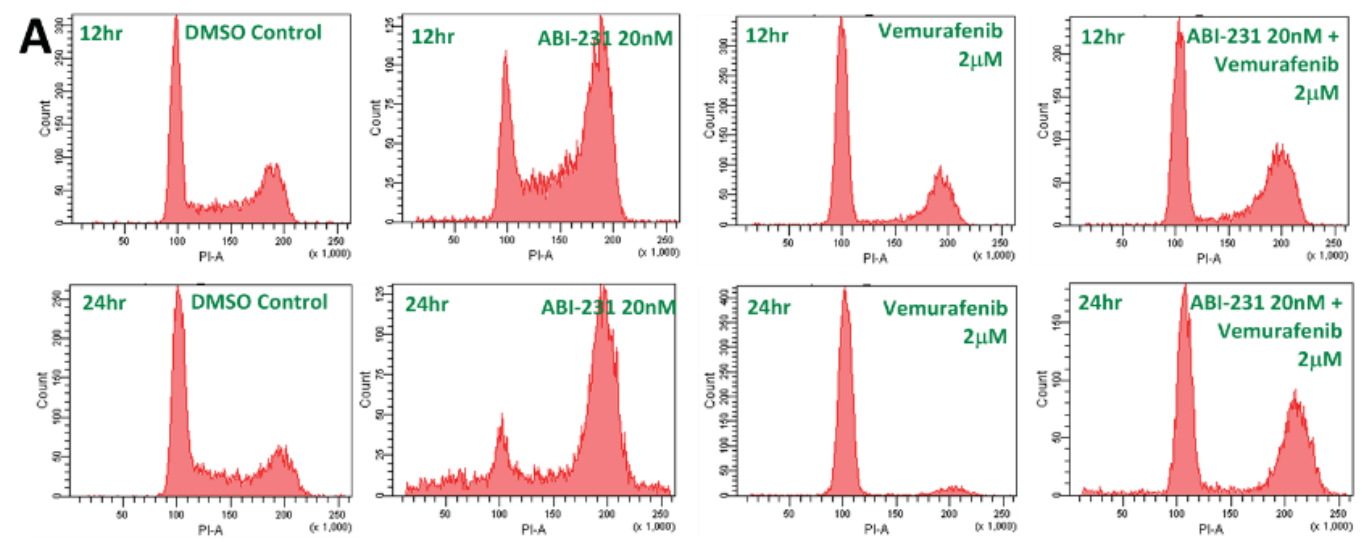

B

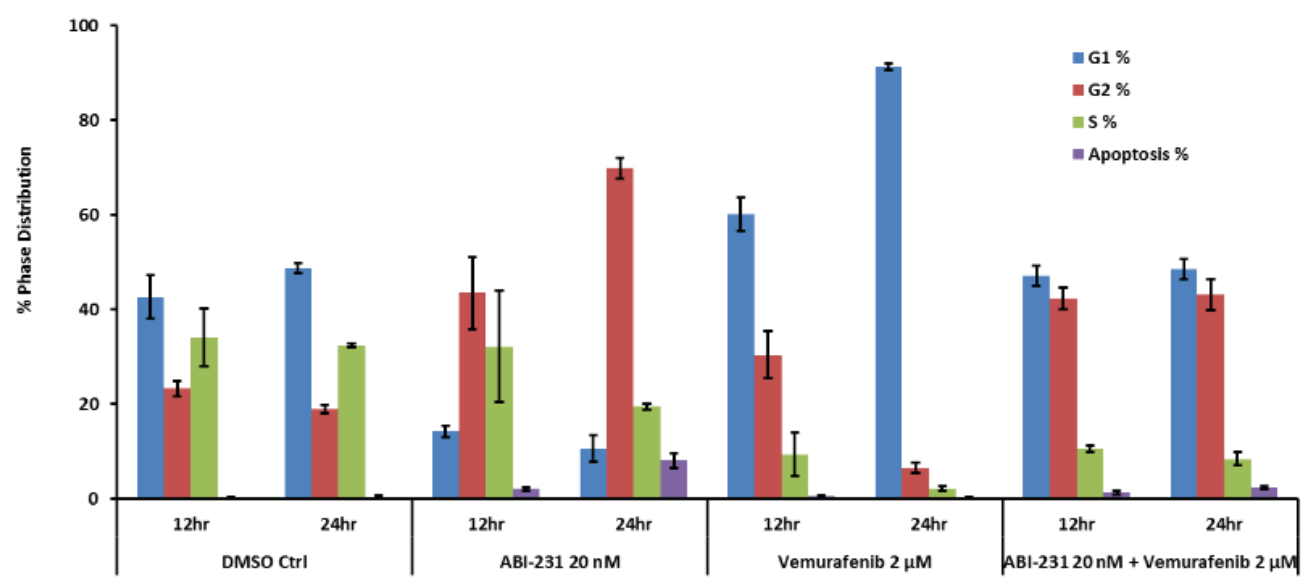

Figure 2-1. Cell cycle analysis of A375 cells incubated with single or combination treatment for $24 \mathrm{~h}(n=3)$

A, representative flow cytometry diagram of cell cycle distribution. B, quantitative results of cell phase distribution. Data is presented by mean \pm S.D. 


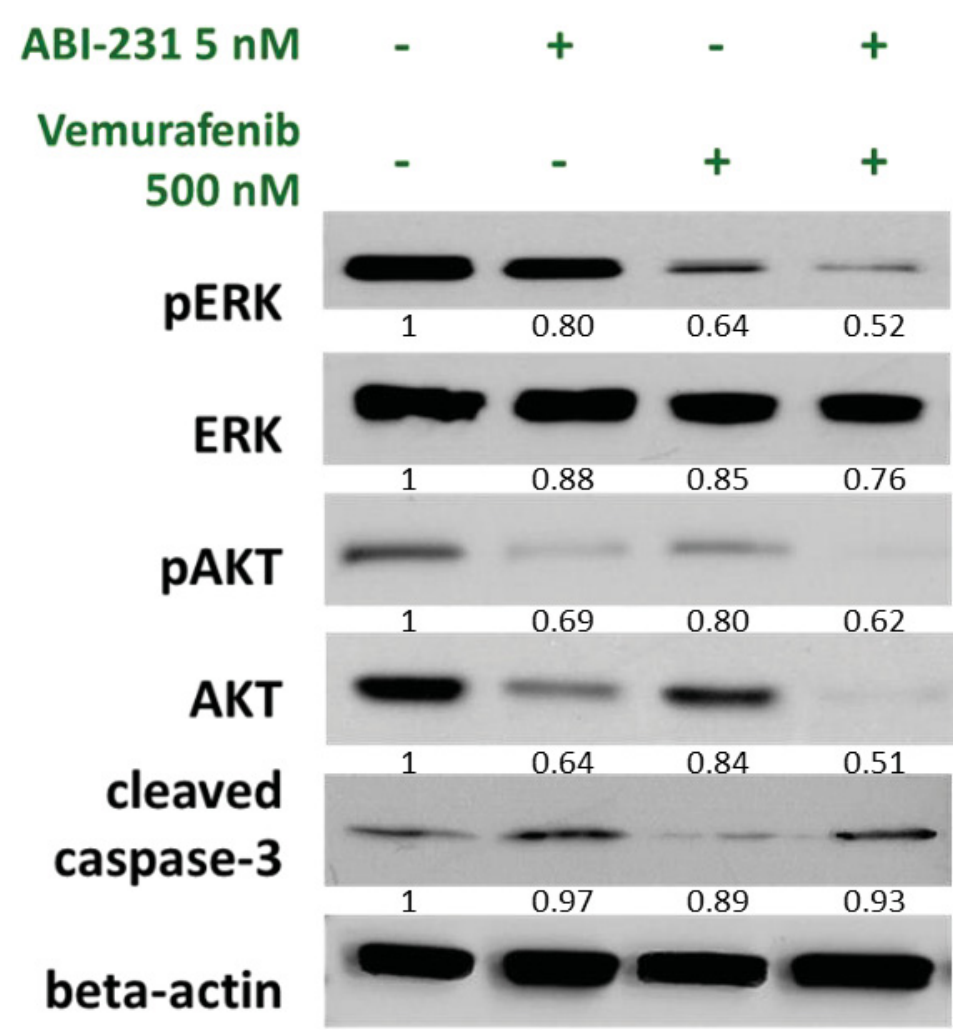

Figure 2-2. Representative images of western blotting results for A375 cell incubated with either single or combination treatment for $24 \mathrm{~h}$

The densitometry data was measured by ImageJ software and normalized according to the reading from beta-actin loading control groups. 
sensitive cell line, the treatment of vemurafenib or combination greatly reduced the level of pERK due to the inhibition of upstream BRAF activity. But ABI-231 single treatment did not affect the level of ERK phosphorylation. Interestingly, AKT phosphorylation level, which is critical in the regulation of cell survival and apoptosis, was reduced in both ABI-231 single treatment and combination treatment group. And the reduction of pAKT in these two groups accompanied with the decrease of total AKT level. This observation indicates that compound ABI-231 as a tubulin polymerization inhibitor exerts its anti-cancer activity partially through the interference of AKT pathway. And the coinhibition of both ERK and AKT phosphorylation generated synergistic anti-proliferation effect in vitro.

\subsubsection{Establishment of Vemurafenib-resistant A375RF21 Melanoma Subline}

Clinically melanoma tumors inevitably relapse only 3 to 6 months after receiving vemurafenib chemotherapy, and therefore we wanted to determine whether the observed synergy will remain effective in vemurafenib-resistant cells. Towards this goal, we established vemurafenib-resistant A375RF21 subline from the parental A375 human melanoma cells by chronic selection following literature reported procedures (28). The isolated resistant A375RF21 cell line steadily increased $\mathrm{IC}_{50}$ values for vemurafenib over 50 fold $(28.9 \pm 0.6 \mu \mathrm{M}$ in A375RF21 cells compare to $0.57 \pm 0.03 \mu \mathrm{M}$ in the parental A375 cells, Figure 2-3).

We performed western blot analyses using both A375 and A375RF21 cells to determine differential protein activations known to result in vemurafenib resistance. As shown in Figure 2-4A, the pERK level in A375RF21 in the presence of $2.5 \mu \mathrm{M}$ vemurafenib (maintenance concentration of its culture medium) does not change, confirming the development of acquired vemurafenib resistance.

The pMEK expression also remains active in A375RF21 cells, indicating their potential cross-resistance to MEK1/2 inhibitors. We confirmed this cross-resistance by incubating cells with two known MEK inhibitors (trametinib and selumetinib, Figure 2-4B and C). The PI3K/AKT pathway was over-activated in A375RF21 cells while no significant changes of RAS, BRAF, ARAF, CRAF levels were observed. These results are consistent with the report of Fei Su et al (28). Interestingly, we found that the level of PDGFR $\beta$ also increased significantly in A375RF21 cells in the presence or absence of the $2.5 \mu \mathrm{M}$ vemurafenib maintenance medium. Both resistant mechanisms that confer drug resistance in A375RF21 cells are well known to exist in vemurafenib-resistant patient tumors.

\subsubsection{Combination of Vemurafenib with ABI-274 Showed Strong Synergies in Vemurafenib-resistant A375RF21 Melanoma Cell Lines}

As shown in Table 2-2, the drug combination study when repeat using A375RF21 cells produced calculated CI values for ABI-231 or ABI-274 in combination with 


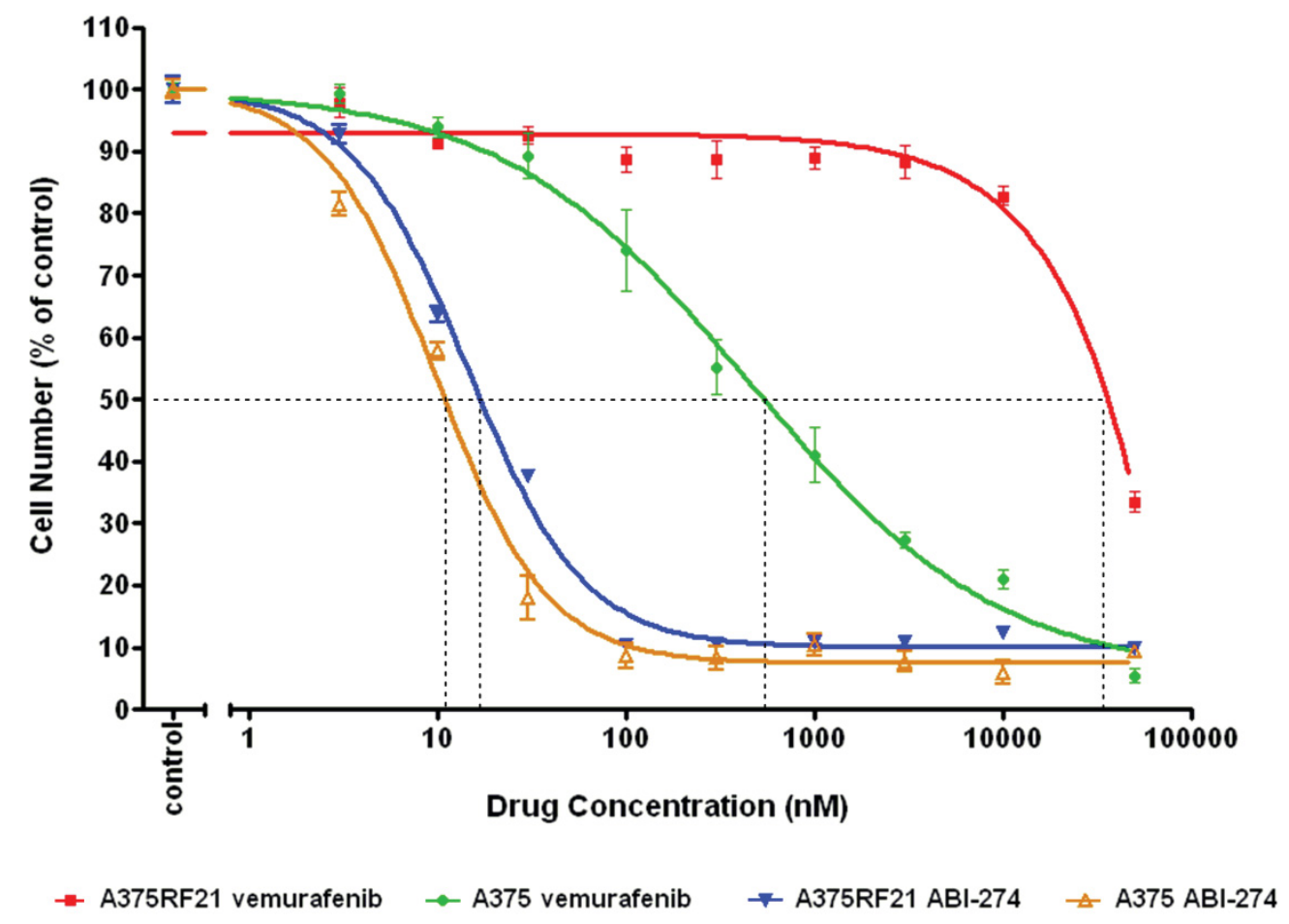

Figure 2-3. Establishment of vemurafenib-resistant A375 melanoma cell line A375RF21 from its parental A375 cell line using chronic selection over 3 months with increasing concentrations of vemurafenib 

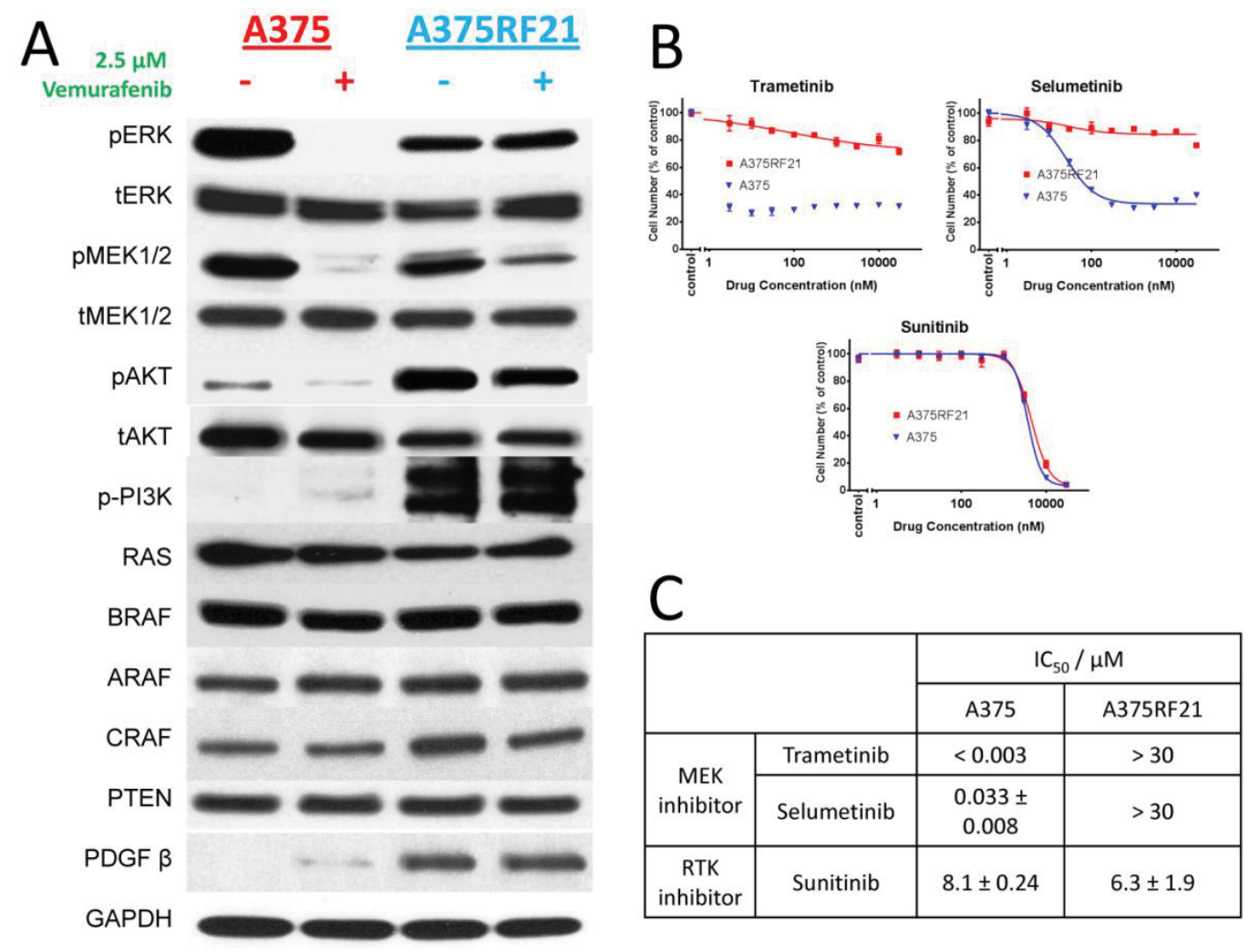

Figure 2-4. Major vemurafenib resistance mechanisms in A375RF21 cells are the over-expression of PDGFR $\beta$ and the activations of the PI3K-AKT pathway A, western blot analyses to compare the differential protein levels in the sensitive parental A375 and the vemurafenib-resistant A375RF21 cells, in the presence or absence of $2.5 \mu \mathrm{M}$ vemurafenib (A375RF21 cell culture maintenance concentration). Cells were incubated with control vehicle or $2.5 \mu \mathrm{M}$ vemurafenib for $24 \mathrm{~h}$. Phospho-PI3K level was determined after 30 minutes stimulantion with $30 \mu \mathrm{M}$ hydrogen peroxide. Graph showed representative results of three independent experiments. B, growth inhibition efficacy of kinase inhibitors determined in MTS assay $(n=4)$ on A375 and A375RF21 cells.

Trametinib and selumenitib are MEK inhibitors while sunitinib (malate salt) is an RTK inhibitor. C, comparison of calculated $\mathrm{IC}_{50}$ values (showed as mean $\pm \mathrm{SD}$ ). 
Table 2-2. Combination of vemurafenib with tubulin inhibitors showed synergistic effect in the vemurafenib-resistant A375RF21 melanoma cell lines

\begin{tabular}{lccc}
\hline $\begin{array}{l}\text { Treatment } \\
+ \text { vemurafenib }\end{array}$ & CI at ED $_{\mathbf{5 0}}$ & CI at ED $_{\mathbf{7 5}}$ & CI at ED $\mathbf{~ 9 0}_{\mathbf{0}}$ \\
\hline ABI-231 & 0.44 & 0.56 & 0.65 \\
ABI-274 & 0.53 & 0.59 & 0.70 \\
Docetaxel & 0.63 & 0.80 & 0.90 \\
Colchicine & 0.84 & 0.94 & 1.36 \\
\hline
\end{tabular}

The combination index $(\mathrm{CI})$ values were calculated based on the results from cell viability MTS assay $(n=5)$. CI $<0.9$ indicates synergism; $0.9 \leq \mathrm{CI} \leq 1.1$ indicates additive effect; $\mathrm{CI}>1.1$ indicates antagonism between the two tested drugs. 
vemurafenib that were all less than 0.9 (range: 0.44-0.70), indicating strong synergy in all concentrations tested. At $\mathrm{ED}_{50}$, all tubulin inhibitors acted in a synergistic manner with vemurafenib. With an increase in drug concentration, the CI values for docetaxel or colchicine groups increased relatively quickly. At the dose of $\mathrm{ED}_{90}$, the combination of docetaxel with vemurafenib was almost additive (CI value as 0.90 ) while the combination effect of colchicine with vemurafenib has reversed to antagonism (CI value as 1.36). Compared with docetaxel or colchicine, ABI compounds showed greater synergy when combined with vemurafenib in the resistant A375RF21 cells. Dose -response curve in Figure 2-5 also clearly showed that in both A375 parental and vemurafenib-resistant A375RF21 subline, the combination of ABI-274 with vemurafenib dramatically increased the anti-proliferation activity comparing with the simple sum of the single treatment.

\subsubsection{Combination of ABI-274 and Vemurafenib Produced Synergistic Cell Cycle Arrests in A375RF21 Cells}

As a tubulin inhibitor binding to the colchicine site, ABI-274 effectively blocks the $\mathrm{G}_{2} / \mathrm{M}$ phase in the parent $\mathrm{A} 375$ cell line in a dose-dependent manner (129). To determine whether a combination of ABI-274 and vemurafenib will arrest vemurafenibresistant cells at different replication phases, we carried out cell cycle analysis in A375RF21 cells. After $24 \mathrm{~h}$ exposure to a compound solution at the indicated concentrations, data in Figure 2-6 clearly indicated synergistic cell cycle arrests. For DMSO controls, $50 \%$ of A375RF21 cells were distributed in $\mathrm{G}_{0} / \mathrm{G}_{1}$ phase and the percentage of cells in $\mathrm{S}$ or $\mathrm{G}_{2} / \mathrm{M}$ phase was $12 \%$ or $32 \%$, correspondingly. For ABI-274 single treated group at a concentration of $20 \mathrm{nM}$, the percentage of cell distributed in $\mathrm{G}_{2} / \mathrm{M}$ phase had accumulated up to $70 \%$. Using vemurafenib as a single agent, to produce similar $G_{0} / G_{1}$ cell cycle arrest in the resistant A375RF21 cell line, the concentration of vemurafenib had to be increased to $30 \mu \mathrm{M}$ or higher, compared with less than $1 \mu \mathrm{M}$ in the parental A375 parental cells. As anticipated, the combination of vemurafenib and ABI274 strongly arrested A375RF21 cells in both $\mathrm{G}_{0} / \mathrm{G}_{1}(48 \%)$ and $\mathrm{G}_{2} / \mathrm{M}(43 \%)$ phase. In addition, this combination treatment generated much more cell debris which indicated an increase in cancer cell apoptosis. Treatment with the combination of vemurafenib and docetaxel produced similar synergistic effects. Flow cytometry analysis with antiphospho-histone $\mathrm{H} 3$ staining (Figure 2-7), which distinguishes the cells between $\mathrm{G}_{2}$ and $\mathrm{M}$ phase, confirmed the same pattern of synergistic cell cycle arrests due to the combination treatments.

\subsubsection{Combination Treatment Induced Significantly Increased Apoptotic and Cell Death in Vemurafenib-resistant Cells}

To understand more clearly the possible cell apoptosis induction effect of the combination treatment, we utilized Annexin V and Propidium Iodide co-staining flow cytometry to differentiate live and apoptotic cells in A375RF21. As expected, single 

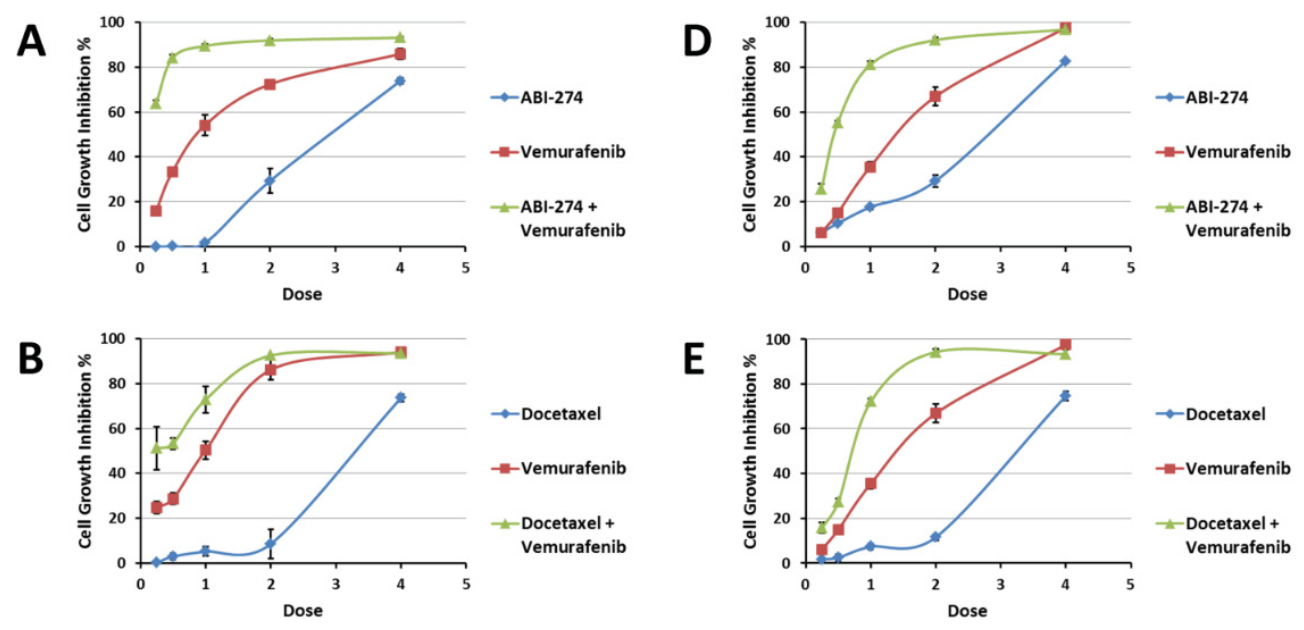

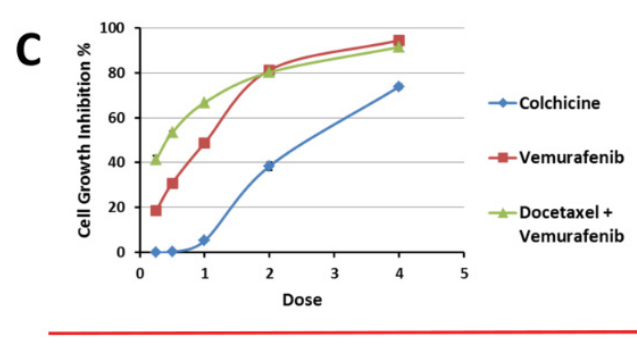

A375

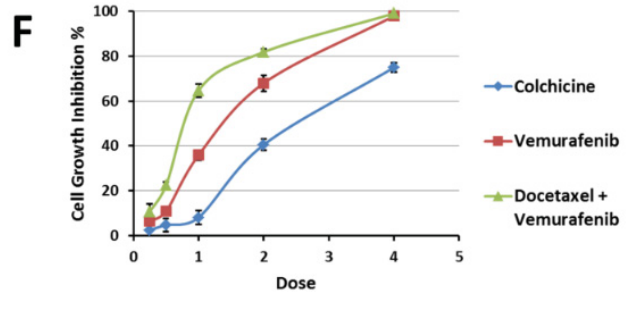

A375RF21

Figure 2-5. In vitro dose-response curves $(n=5)$ of each combination in A375 and A375RF21 cells

$\mathrm{X}$-axis of each plot is the dose density regarding $\mathrm{IC}_{50}$ concentrations of drug $\mathrm{A}$ or $\mathrm{B}$ on $\mathrm{A} 375$ or A375RF21 cells in an A+B combination treatment. Figure $\mathrm{A}$ to $\mathrm{C}$ is the data from A375 cells and Figure D to $F$ is the data from A375RF21 cells. 

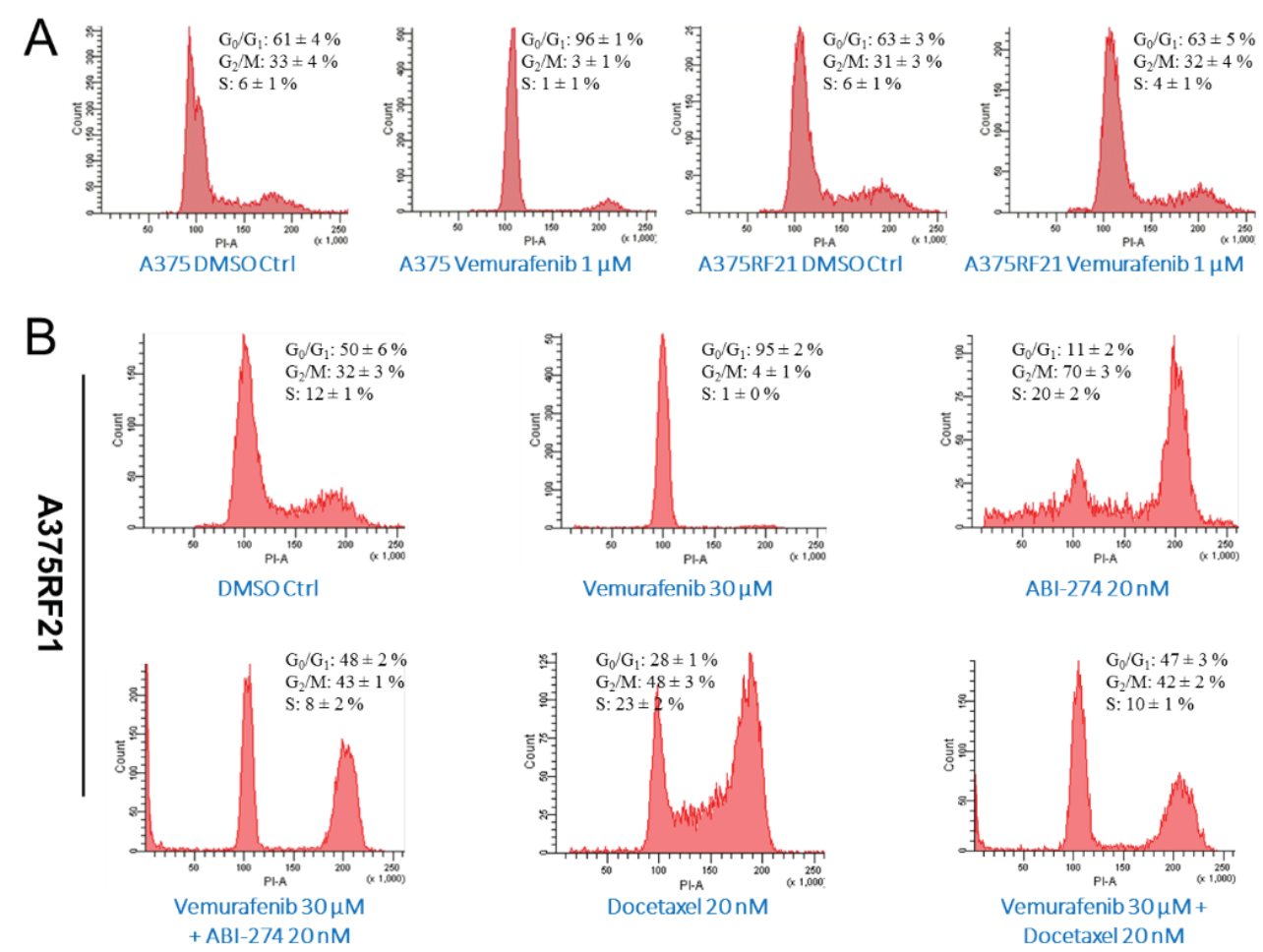

Figure 2-6. Cell cycle analysis for combination of ABI-274 with vemurafenib ( $n=$ 4)

A, A375 or A375RF21 cells treated with $1 \mu \mathrm{M}$ vemurafenib for $24 \mathrm{~h}$ and compared with the DMSO control group. Vemurafenib at $1 \mu \mathrm{M}$ effectively arrested A375 cells at $\mathrm{G}_{0} / \mathrm{G}_{1}$ phases but could not arrest resistant A375RF21 cells. B, A375RF21 cells treated with DMSO, $30 \mu \mathrm{M}$ vemurafenib, $20 \mathrm{nM}$ ABI-274, $20 \mathrm{nM}$ docetaxel and the combinations for 24h. ABI-274 and docetaxel induced $\mathrm{G}_{2} / \mathrm{M}$ arrest in A375RF21 cells and their combinations with vemurafenib arrested cells in $\mathrm{G}_{1} / \mathrm{G}_{2} / \mathrm{M}$ phases. 

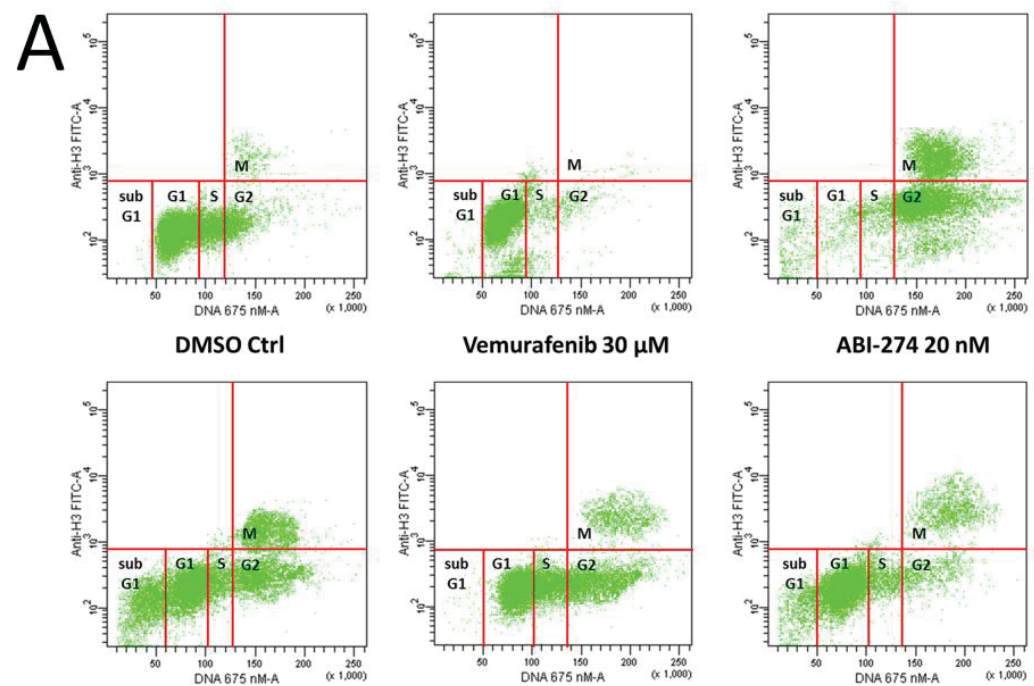

Vemurafenib $30 \mu \mathrm{M}$

ABI-274 $20 \mathrm{nM}$

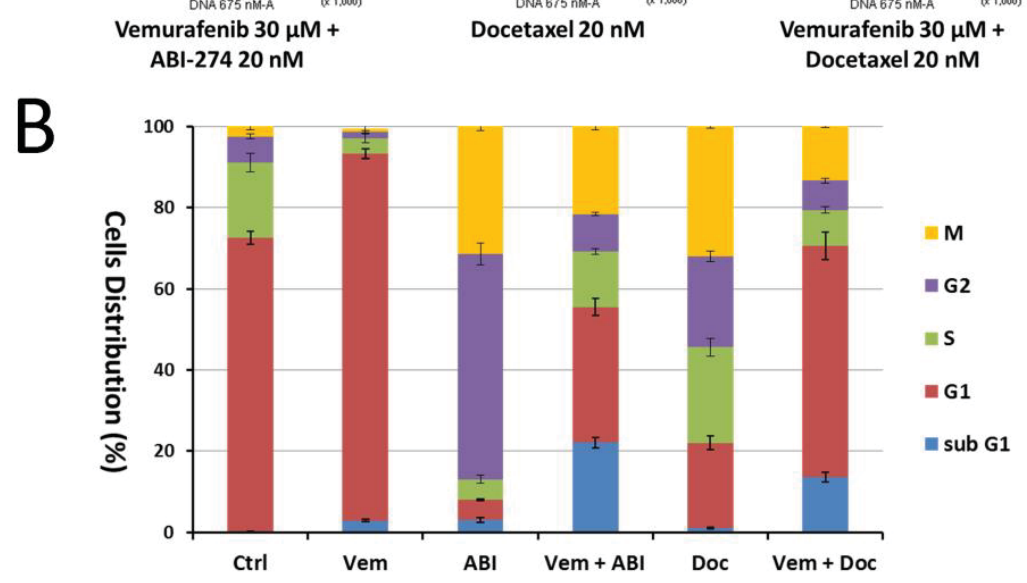

Figure 2-7. Anti-phospho-histone H3 and PI (propidium iodide) bivariate staining cell cycle analysis on vemurafenib-resistant A375RF21 cells

A, representative diagrams illustrated the cell distribution. The red lines were defined manually to show how the cell cycle phase distribution had been calculated accordingly. $B$, quantification data (mean \pm S.D.) for cell cycle phases distribution. Ctrl: $5 \%$ DMSO; Vem: vemurafenib $30 \mu \mathrm{M}$; ABI: ABI-274 20 nM; Vem + ABI: vemurafeib $30 \mu \mathrm{M}+$ ABI-274 20 nM; Doc: docetaxel 20 nM; Vem + Doc: vemurafeib $30 \mu \mathrm{M}+$ docetaxel 20 nM. 
agent treatment produced only moderate effects on inducing cell apoptosis at tested concentrations; in contrast, the combination treatment groups significantly enhanced the apoptosis (Figure 2-8A). As shown in Figure 2-8B, in which we quantified the percentage sum of cell distributed in Q1 (early apoptosis), Q2 (apoptosis) and Q4 (dead cells), the combination of ABI-274 and vemurafenib resulted in $50 \pm 7.6 \%$ of counted cell apoptosis or death, which is much higher than the simple sum of apoptotic percentages in two single agent treatment groups $(11.8 \pm 3.0 \%$ for ABI-274 and $11.9 \pm$ $3.5 \%$ for vemurafenib). A similar synergy effect of apoptosis induction was also observed in the combination treatment group containing docetaxel $(6.4 \pm 3.0 \%$ for docetaxel group, $38.1 \pm 2.6 \%$ for combination).

\subsubsection{Combination Mitigates Acquired Vemurafenib Resistance by Down-regulating pAKT or Total AKT and Activating Apoptosis Cascades}

It has been established that ABI-274 targets tubulin polymerization $(125,126)$ and vemurafenib targets $\mathrm{BRAF}^{\mathrm{V} 600 \mathrm{E}}$. As the first approach to understand responsible molecular mechanisms leading to this strong synergistic combination, we investigated whether the synergy is mediated through potentiation of the direct target of ABI-274 or vemurafenib. As shown in Figure 2-9 vemurafenib did not have any effect on tubulin polymerization, even at a high concentration of $20 \mu \mathrm{M}$. The addition of vemurafenib to ABI-274 at most marginally increased the inhibition of tubulin polymerization compared with the single agent ABI-274. The inhibition of tubulin polymerization in the combination treatment was exclusively contributed by ABI-274, suggesting the synergistic combination was not mediated through potentiation of the direct target inhibition for ABI-274.

Next we determined using western blotting whether the combination has any effects on $\mathrm{pERK}$, the hallmark of BRAF ${ }^{\mathrm{V} 600 \mathrm{E}}$ inhibition by vemurafenib. Figure 2-10A revealed that either ABI-274 or the combination treatments had no obvious effect on pERK or total ERK level after 48h incubation with A375RF21 resistant cells. Replacing ABI-274 with another tubulin inhibitor, docetaxel, produced similar results in Figure 2-10A). Therefore, the synergistic combination was unlikely through potentiation of the inhibition of $\mathrm{BRAF}^{\mathrm{V} 600 \mathrm{E}}$.

Recently, Fei Su et al reported that pAKT levels were increased in A375 vemurafenib-resistant clones compared with their parental vemurafenib-sensitive cells (28). Since A375RF21 cells also have strong pAKT activation (Figure 2-10A), we hypothesize that the combination treatment may produce its strong synergy through down-regulating activities in the AKT pathway in vemurafenib-resistant A375RF21 cells. As shown in Figure 2-10A, both pAKT and total AKT (tAKT) were greatly reduced in single-agent ABI-274 or its combination treatment group after $48 \mathrm{~h}$ incubation, suggesting that the synergistic anti-proliferation might be mediated by simultaneously targeting both ERK and AKT phosphorylation. Docetaxel also reduced the pAKT and tAKT expression and had similar effects in its combined treatment with vemurafenib. For example, in addition to the obvious reduction of tAKT levels, the combination of ABI-274 and 

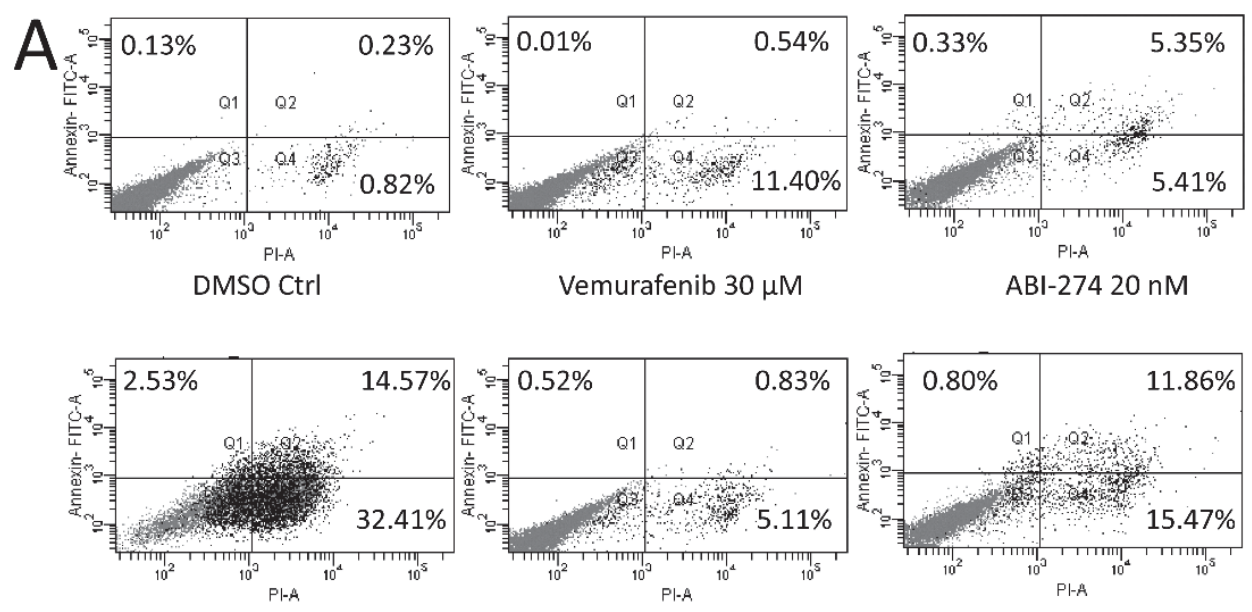

Vemurafenib $30 \mu \mathrm{M}+$ $\mathrm{ABI}-27420 \mathrm{nM}$

Docetaxel $20 \mathrm{nM}$

B

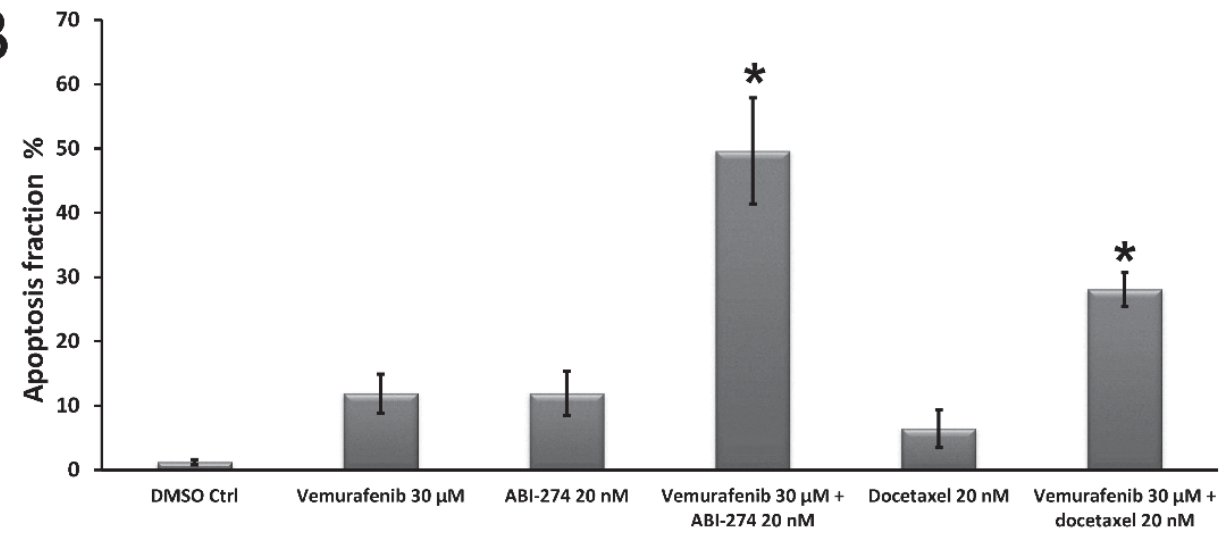

Figure 2-8. Combination of tubulin inhibitors with vemurafenib synergistically increased proportion of cell apoptosis or death in resistant A375RF21 cells A, representative quadrant diagrams illustrated the cell distribution in Q1 (early apoptosis), Q2 (apoptosis), Q3 (live), and Q4 (dead). Cell clusters with high-SSC (side scatter)/ low-FSC (forward scatter) cyto-morphological profiles were colored in black. There was no back-gating difference between grey and black populations. B, apoptosis fraction was calculated by adding distribution percentage in Q1, Q2 and Q4 together. Drug combinations induced significantly higher $\left({ }^{*} P<0.05\right)$ portion of apoptosis compared with simple sum of apoptosis fraction in two single agent treatment groups. 


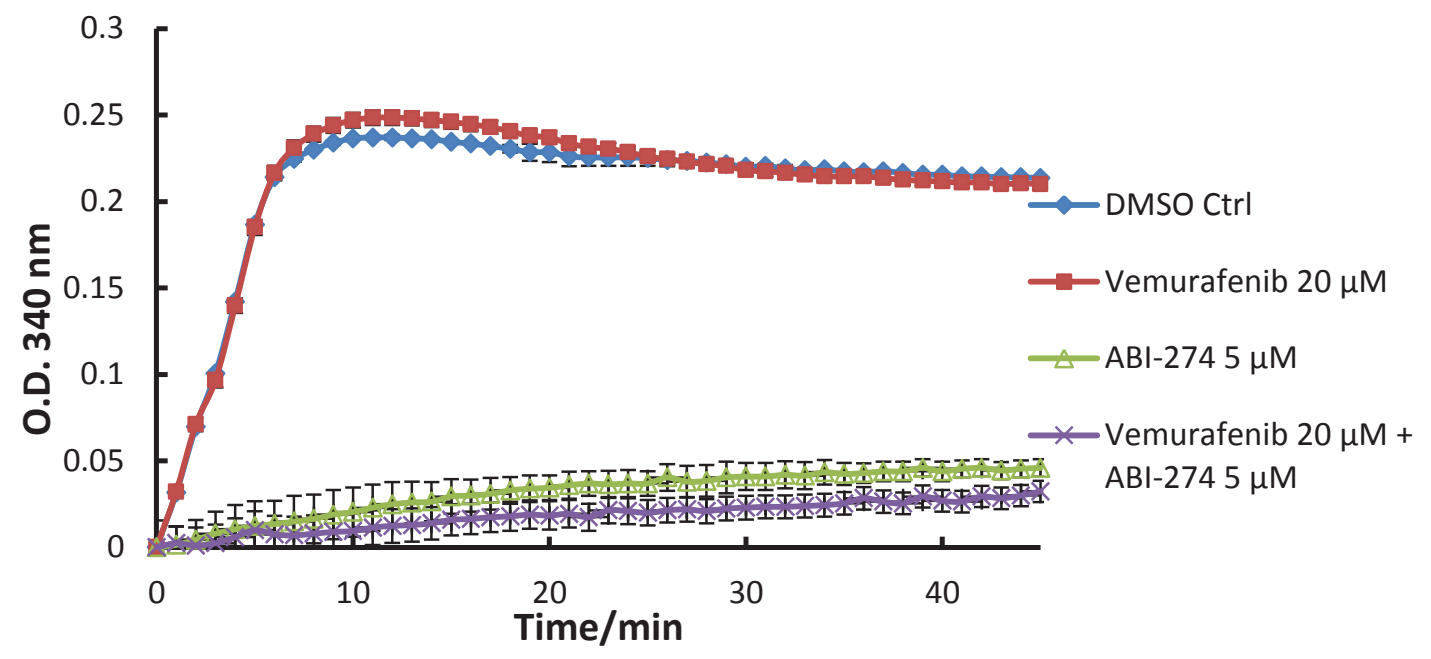

Figure 2-9. Effect of single agent and combination treatment on purified-protein based tubulin polymerization assay $(n=3)$

Vemurafenib at $20 \mu \mathrm{M}$ did not significantly influence tubulin polymerization compared with DMSO control group. The tubulin polymerization inhibition effect in the combination treated group was solely contributed by ABI-274. 


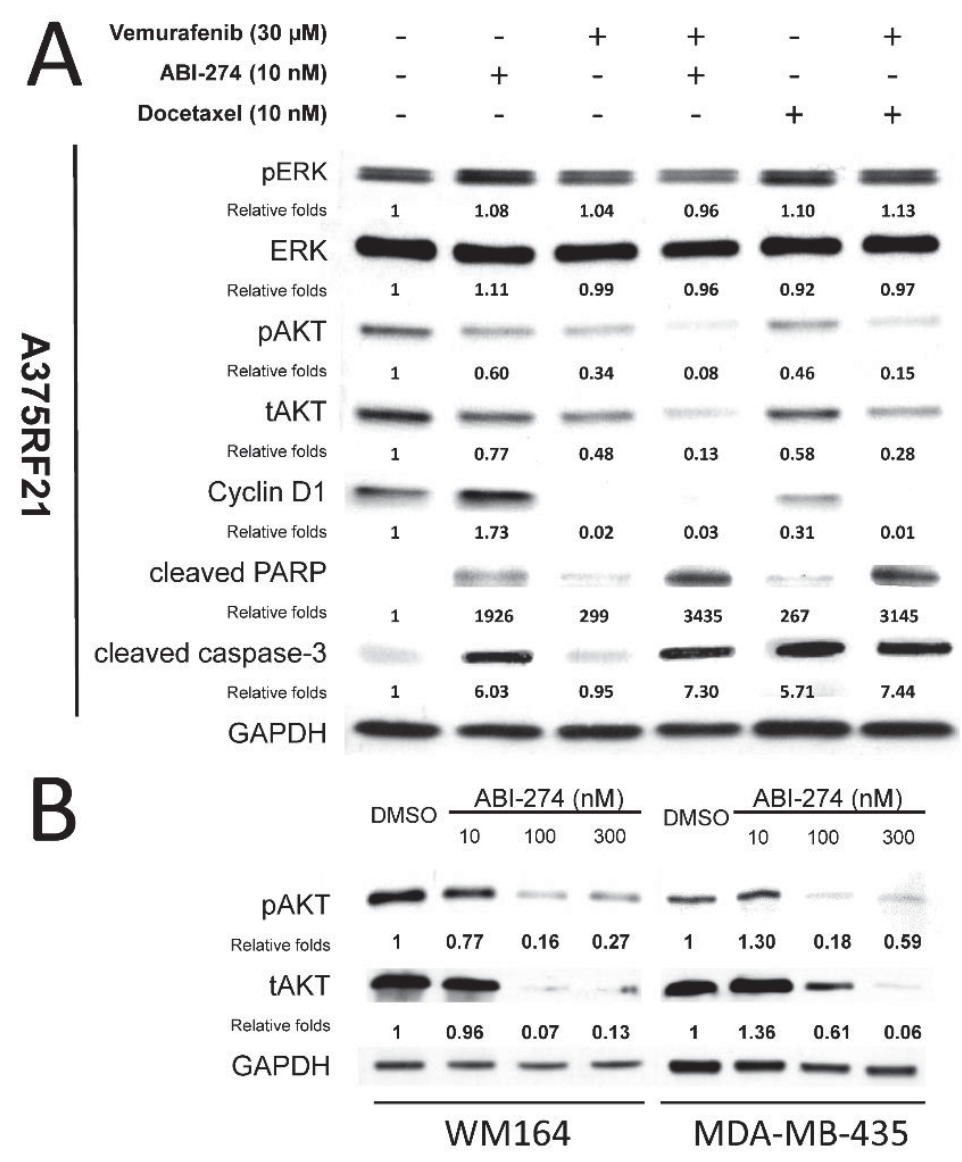

Figure 2-10. Western blot analysis with indicated antibodies on lysate of A375RF21 (A), MDA-MB-435 and WM164 cells (B) after 48h treatment while GAPDH was used as a loading control

A, while the indicated combination treatment only caused moderate decreasing of $p$-ERK level, they largely inhibited the AKT phosphorylation and increased the level of apoptotic markers including cleaved PARP and cleaved caspase-3. B, ABI-274 also displayed AKT knock-out effects in other two BRAF ${ }^{\mathrm{V} 600 \mathrm{E}}$ mutant human melanoma cell lines, MDAMB-435 and WM164. 
vemurafenib reduced the level of pAKT to $61 \%$ relative to tAKT (calculated from the quantified relative folds of lane density: $0.08 / 0.13 \times 100 \%$ ) while the single-agent treatment only reduced the levels of pAKT to $77 \%$ (ABI-274, $0.6 / 0.77 \times 10 \%$ ) and $70 \%$ (vemurafenib, $0.34 / 0.48 \times 100 \%$ ) relative to the corresponding levels of tAKT, respectively.

The strong dose-dependent pAKT/tAKT inhibition effects of ABI-274 were further confirmed in other two BRAF ${ }^{\mathrm{V} 600 \mathrm{E}}$ mutant cell lines, WM164 and MDA-MB-435 (Figure 2-10B). Furthermore, since the highly phosphorylated AKT level is associated with upregulated PDGFR $\beta / P I 3 K$ in A375RF21 cells (Figure 2-4A), we continued to check the changes of PDGFR $\beta / \mathrm{PI} 3 \mathrm{~K}$ levels in response to both single and combination treatment. Representative results in Figure 2-11 revealed that after 24hr incubation, ABI274 or docetaxel at the concentration of $20 \mathrm{nM}$ generally wiped out the PDGF $\beta$ and leaded to the down-regulation of PI3K level in A375RF21 cells. As a result, the AKT upstream PDGFR $\beta / P I 3 K$ levels were greatly reduced in combination treatment groups, although vemurafenib single treatment did not significantly affect the PDGFR $\beta / \mathrm{PI} 3 \mathrm{~K}$. Therefore, our data indicates that ABI-274 or docetaxel down-regulates $\mathrm{pAKT} / \mathrm{tAKT}$ activity through the inhibition of PDGFR $\beta / \mathrm{PI} 3 \mathrm{~K}$ pathway.

In the vemurafenib-resistant cells (Figure 2-10A), decreased cyclin D1 levels in vemurafenib and the combination treatment groups indicated inhibitions on $\mathrm{G}_{0} / \mathrm{G}_{1}$ cellcycle progression. Apoptosis markers, cleaved PARP and caspase-3, were highly induced by tubulin inhibitors while vemurafenib slightly increased their expression. This result is consistent with our observation in apoptosis detection experiment.

\subsubsection{Combination of Vemurafenib and ABI-274 Synergistically Suppresses Vemurafenib-resistant Tumor Growth In Vivo}

To evaluate whether the strong synergy observed against A375RF21 cells in vitro could be transferred to vemurafenib-resistant tumors in vivo, we compared the effect of combination efficacy on tumor growth with single agent treatments. We previously established that ABI-274 is effective in suppressing parental A375 melanoma tumor growth in vivo at a dose of $25 \mathrm{mg} / \mathrm{kg}$ (129). A pilot study showed that vemurafenibresistant A375RF21 cells had similar growth kinetics to their parental A375 cells in the absence of drug treatment. We reduced the dose of ABI-274 to $10 \mathrm{mg} / \mathrm{kg}$ in the current study in order to avoid any potentially unexpected toxicity due to its combination with vemurafenib.

As shown in Figure 2-12 and Table 2-3, vemurafenib (20 mg/kg) mono-therapy only achieved minimal (22.65\%) TGI and ABI-274 (10 mg/kg) by itself resulted in slightly better TGI at $38.12 \%$ in this vemurafenib-resistant tumor model; in contrast, their combination treatment significantly enhanced tumor inhibition to $88.56 \%$ after 3 -week treatment (Figure 2-12A, B and Table 2-3). Three out of the seven mice that received combination therapy were kept for additional 7 days without further treatment, 


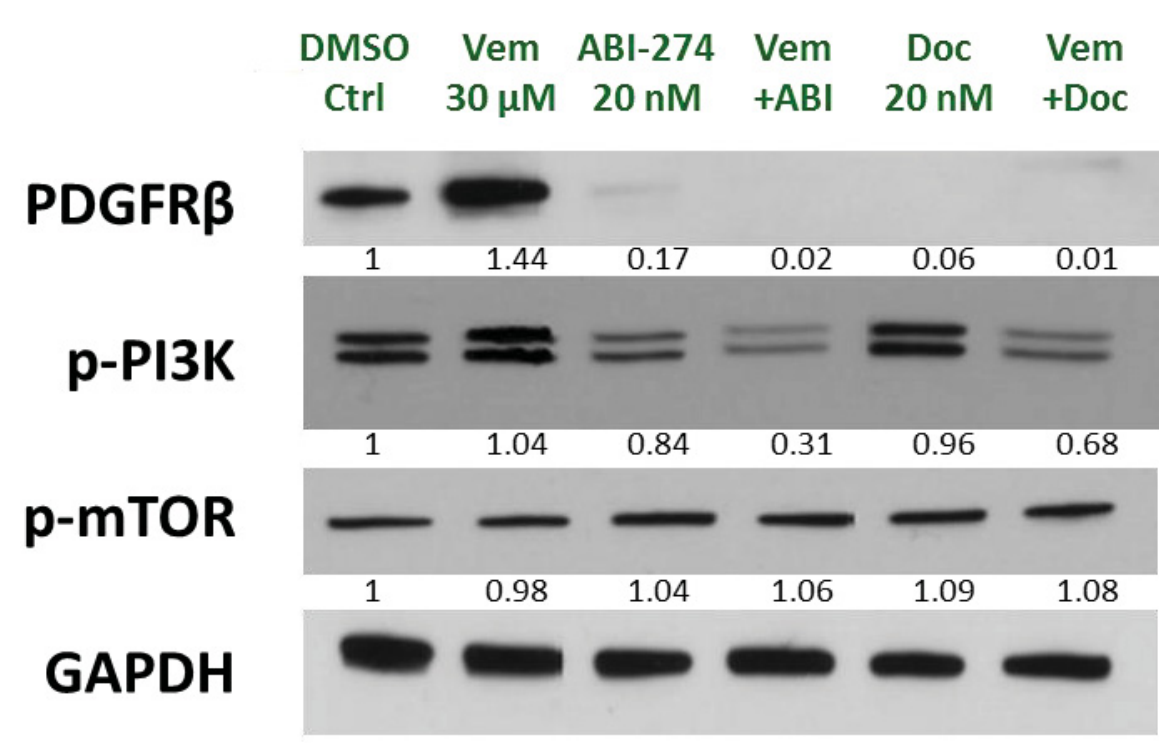

Figure 2-11. Western blotting analysis for cell lysates of A375RF21 incubated with indicated treatment for $24 \mathrm{~h}$

The densitometry data was measured by ImageJ software and normalized according to the reading from beta-actin loading control groups. 

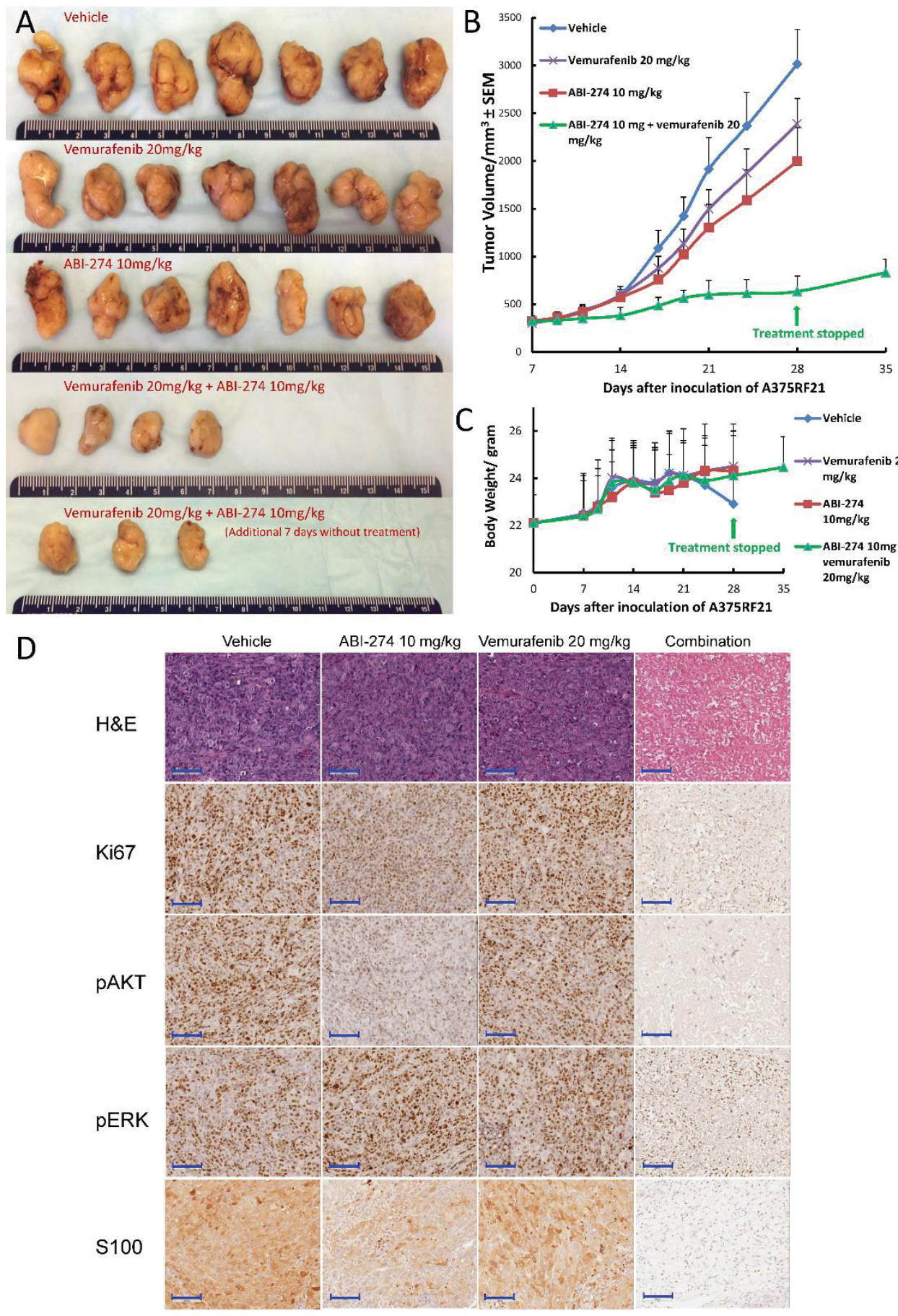

Figure 2-12. In vivo combination of vemurafenib and ABI-274 in the resistant A375RF21 xenograft model $(n=7)$

A, pictures of isolated tumor tissue. B, tumor volume growth curve. C, mice body weight versus time plot. D, representative immunohistochemistry images for H\&E, Ki67, pAKT, pERK and S100 staining of xenograft tumor tissue sections after three weeks of single agent or combination treatment. The scale bar (yellow or blue) in each image represents $100 \mu \mathrm{m}$. 
Table 2-3. Tumor growth inhibition (TGI) and tumor weight comparison for in vivo combination of vemurafenib and ABI-274 in the resistant A375RF21 xenograft model $(n=7)$

\begin{tabular}{lcc}
\hline Treatment group & TGI (\%) & $\begin{array}{c}\text { Tumor weight } \\
\text { (gram) }\end{array}$ \\
\hline Vehicle & - & $2.48 \pm 0.27$ \\
$\mathrm{ABI}-27410 \mathrm{mg} / \mathrm{kg}$ & $38.12 \pm 6.14$ & $1.77 \pm 0.11$ \\
Vemurafenib $20 \mathrm{mg} / \mathrm{kg}$ & $22.65 \pm 8.31$ & $2.05 \pm 0.14$ \\
Vemurafenib 20 mg/kg + ABI-274 & $88.56 \pm 3.57^{*}$ & $0.77 \pm 0.17$ \\
$\begin{array}{l}\text { Vemg/kg (after 3 weeks of treatment) } \\
\text { Vemurafenib 20 mg/kg + ABI-274 }\end{array}$ & $81.27 \pm 5.52^{*}$ & $0.90 \pm 0.11$ \\
$\begin{array}{l}10 \mathrm{mg} / \mathrm{kg} \text { (additional 7 days without } \\
\text { treatment) }\end{array}$ & & \\
\hline
\end{tabular}

The combination of ABI-274 at $10 \mathrm{mg} / \mathrm{kg}$ and vemurafenib at $20 \mathrm{mg} / \mathrm{kg}$ achieved greater antitumor activity (TGI) compared with the simple sum of TGI in two single agent treatment groups $(* P<0.05)$. The synergistic tumor inhibition sustained after additional one week without further treatment $\left({ }^{*} P<0.05\right)$. 
and showed no significant $(P=0.2857)$ tumor relapse and maintained $81.27 \%$ tumor suppression. During the entire experiment, no mice in the four groups lost body weight by more than 10\% (Figure 2-12C), indicating the absence of gross toxicity for these treatments. When mice were euthanized, major organs including brain, hearts, kidney, liver, spleen, and lungs were isolated and were submitted for pathological analysis. No abnormalities were observed on these organs. Collectively, these results strongly indicated that this combination treatment effectively helped overcoming the acquired resistance to vemurafenib in A375RF21 melanoma model and further confirmed the synergistic anti-proliferation effects observed in vitro.

To determine whether the down-regulation of AKT signaling by combination treatment observed in vitro also functions in vivo, we performed immunohistochemistry analyses on tumor sections from all the experimental groups. We also determined the activity in the ERK pathway and assessed the proliferation level indicated by cell marker Ki-67 in tumor sections. As shown in Figure 2-12E, the improved pathway and proliferation inhibition in the combination treatment groups corresponded well with overall tumor response TGI results. ERK and AKT phosphorylation together with Ki67 expression levels in either nucleus or cytoplasm were largely reduced in the combination treatment group. Furthermore, wide area of background pink colored from Matrigel in $H \& E$ staining for tumor sections in the combination treatment group indicated that very few tumor cells, if any, remained after combination treatment. The significant reduction of melanoma cells in the combination treatment group was further confirmed by the reduced density in S100 immunostains (Figure 2-12D).

Since the results presented in Figure 2-12 and Table 2-3 were promising but did not seem to result in tumor regression, we repeated the experiment by increasing the dose by $50 \%$ for both ABI-274 and vemurafenib. The results are shown in Figure 2-13 and Table 2-4. There is a slight increase of efficacy for vemurafenib (TGI of $22.65 \%$ at 20 $\mathrm{mg} / \mathrm{kg}$ vs. $28.10 \%$ at $30 \mathrm{mg} / \mathrm{kg}$ ), and there is substantial increase of efficacy for ABI-274 (TGI of $38.12 \%$ at $10 \mathrm{mg} / \mathrm{kg}$ vs. $72.72 \%$ at $15 \mathrm{mg} / \mathrm{kg}$ ). Moderate tumor regression (44.9 $\%$ ) on the combination treatment group with the increase drug dose was apparent as shown in Figure 2-13B. Collectively, these data provided the first convincing evidence that the combinations of novel tubulin inhibitors, such as ABI-274 with vemurafenib, are likely to overcome the acquired resistance to vemurafenib for melanoma patients having $\mathrm{BRAF}^{\mathrm{V} 600 \mathrm{E}}$ mutation.

\subsubsection{Establishment of Vemurafenib-resistant MDA-MB-435 VemR Melanoma Subline}

To further expand the panel of vemurafenib-resistant cell model that can be used to evaluate the efficacy and mechanism of our combination treatment, we chronically selected one additional melanoma cell line with acquired resistance to vemurafenib, MDA-MB-435 VemR cells, following similar procedure that we used to generate A375RF21 cell line. 

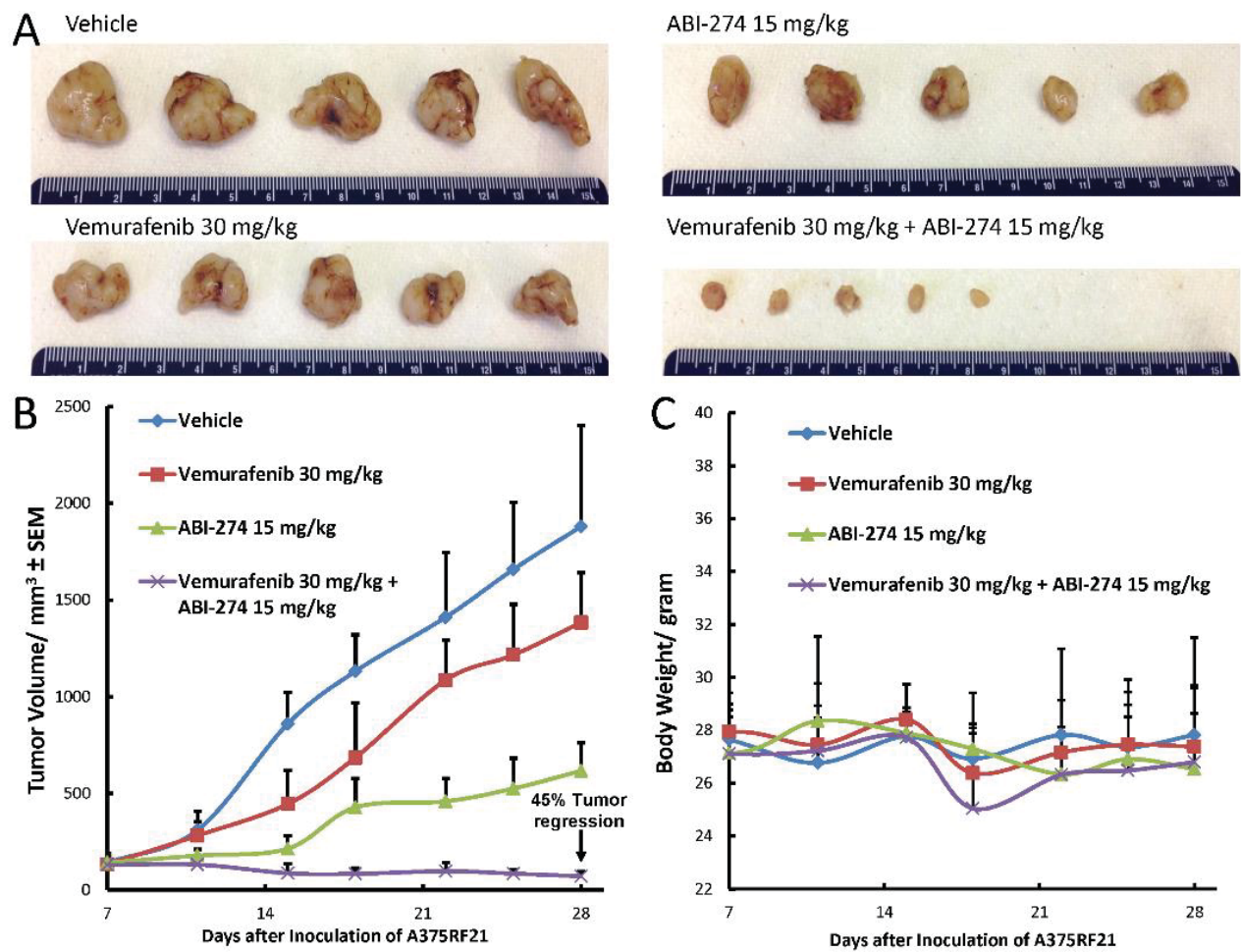

Vemurafenib $30 \mathrm{mg} / \mathrm{kg}+\mathrm{ABI}-27415 \mathrm{mg} / \mathrm{kg}$
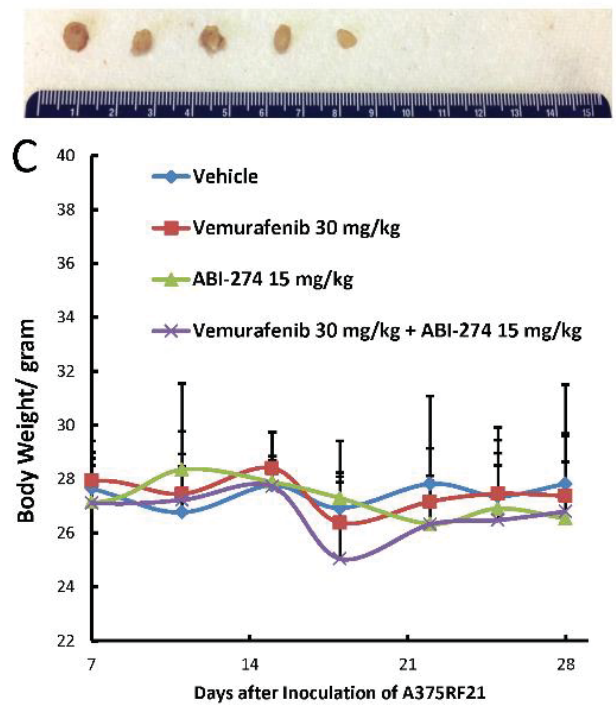

Figure 2-13. In vivo combination of high dose vemurafenib $(30 \mathrm{mg} / \mathrm{kg})$ and $\mathrm{ABI}-$ $274(15 \mathrm{mg} / \mathrm{kg})$ in the A375RF21 xenograft model $(n=5)$

A, pictures of isolated tumor tissue. B, tumor volume growth curve. C, mice body weight versus time plot. Combination of ABI-274 and vemurafenib at this dose achieved about $45 \%$ of tumor regression. 
Table 2-4. Tumor growth inhibition (TGI) and tumor weight comparison for in vivo combination of vemurafenib $(30 \mathrm{mg} / \mathrm{kg})$ and $\mathrm{ABI}-274(15 \mathrm{mg} / \mathrm{kg})$ in the resistant A375RF21 xenograft model $(n=5)$

\begin{tabular}{lcc}
\hline Treatment group & TGI (\%) & $\begin{array}{c}\text { Tumor weight } \\
\text { (gram) }\end{array}$ \\
\hline Vehicle & - & $1.60 \pm 0.22$ \\
Vemurafenib $30 \mathrm{mg} / \mathrm{kg}$ & $28.10 \pm 4.81$ & $1.05 \pm 0.21$ \\
ABI-274 $15 \mathrm{mg} / \mathrm{kg}$ & $72.72 \pm 8.29$ & $0.51 \pm 0.12$ \\
Vemurafenib $30 \mathrm{mg} / \mathrm{kg}+\mathrm{ABI}-274$ & $103.38 \pm 1.42$ & $0.08 \pm 0.03$ \\
$15 \mathrm{mg} / \mathrm{kg}$ & & \\
\hline
\end{tabular}


As determined by MTS assay, the established MDA-MB-435 VemR subline is resistant to both vemurafenib and another recently approved BRAF inhibitor, dabrafenib. $\mathrm{IC}_{50}$ of vemurafenib increased over 14 folds, from $678 \pm 24 \mathrm{nM}$ in MDA-MB-435 cells to $9.82 \pm 0.04 \mu \mathrm{M}$ in MDA-MB-435 VemR cells. And $\mathrm{IC}_{50}$ of dabrafenib increased over 50 folds, from $4 \pm 1 \mathrm{nM}$ in MDA-MB-435 cells to $211 \pm 13 \mathrm{nM}$ in MDA-MB-435 VemR cells. However, as shown in Figure 2-14A, MDA-MB-435 VemR cell was not crossresistant to MEK inhibitors trametinib. This phenotype indicated that MDA-MB-435 VemR had developed resistance to BRAF inhibitor through mechanism different from A375RF21 cells. In fact, western blotting analysis (Figure 2-14B) revealed that PDGFR $\beta$ level in MDA-MB-435 VemR cells was largely decreased comparing with that in MDAMB-435 parental cells, which is distinct from A375RF21 cells. Interestingly, although no significant activation of AKT/mTOR was observed in MDA-MB-435 VemR cells, MEK phosphorylation level maintained in the resistant cells when it was treated by vemurafenib. Therefore, MDA-MB-435 VemR adopted the resistance to BRAF inhibitor through MEK activation mediated mechanism.

\subsubsection{Combination of Tubulin Inhibitor with BRAF Inhibitor Showed Synergistic Anti-proliferation Effects in Expanded Panel of Vemurafenib or Trametinib Resistant Cell Lines}

Due to the complexity of BRAF inhibition resistant mechanism and the heterogeneity of melanoma tumor, the efficacy of combination therapy need to be tested on expanded panel of BRAF resistant cell lines other than A375RF21. In our antiproliferation study, combination of tubulin inhibitor with BRAF inhibitor continuously displayed synergistic effect in MDA-MB-435 VemR, 451LuMR and WM983B BR cells. Among them, 451LuMR and WM983B BR cells were established by Dr. Mennhard Herlyn's lab through chronical exposing BRAF ${ }^{\mathrm{V} 600 \mathrm{E}}$ human melanoma $451 \mathrm{Lu}$ and WM983B cells to trametinib or vemurafenib, accordingly. These cells are found to cross resistant to vemurafenib, dabrafenib and trametinib(131). Combination index as shown in Table 2-5 indicated that ABI-274 combined with either vemurafenib or dabrafenib displayed strong in vitro synergistic effect in MDA-MB-435 VemR cells. In 451LuMR and WM983B BR cells, combination therapy of ABI-274 and vemurafenib showed sustained synergistic growth inhibition, while the combination of vemurafenib with trametinib only had additive anti-proliferation activity.

\subsection{Discussion}

Although vemurafenib, the first drug approved for melanoma patients harboring $\mathrm{BRAF}^{\mathrm{V} 600 \mathrm{E}}$ mutation, showed remarkable responses in initial therapy, almost all patients taking this drug developed resistance to vemurafenib within a few months (132). Understanding the underlying mechanisms of either primary or acquired resistance and developing suitable combination strategies could provide more effective ways to overcome such resistance. There is a rich literature in both preclinical studies and clinical trials to search for effective combination of vemurafenib with other agents in order to 


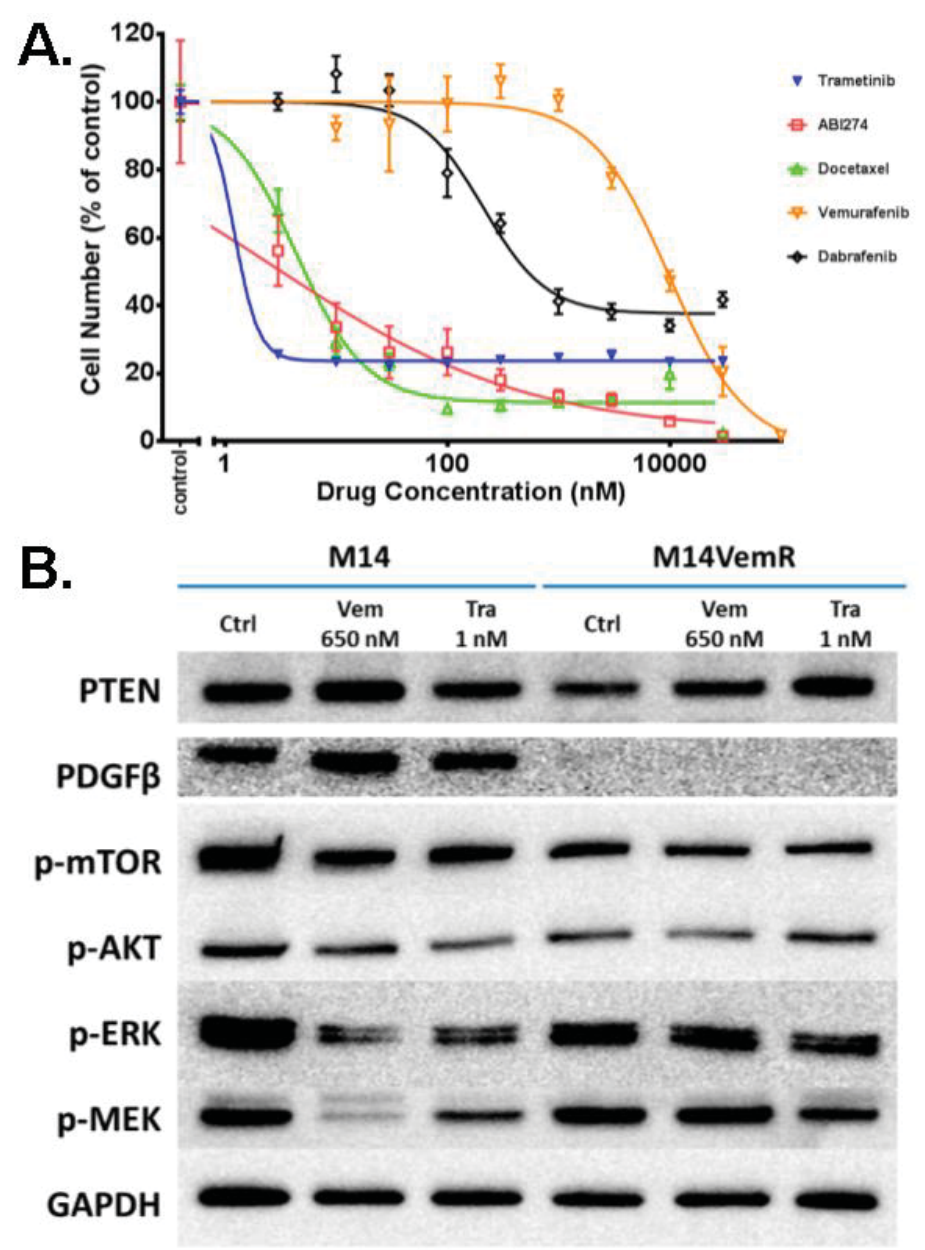

Figure 2-14. Establishment of BRAF inhibitor resistant MDA-MB-435 VemR subline with chronically selection in vitro

A, dose-growth inhibition curves of MDA-MB-435 VemR cells when treated by BRAF inhibitor, MEK inhibitor or tubulin inhibitor. Data was determined by MTS assay in vitro $(n=4)$. B, western blotting analysis revealed that MDA-MB-435 developed resistance to BRAF inhibitors through MEK activation mediated mechanism. 
Table 2-5. Combination of BRAF inhibitors with ABI-274 showed synergistic effect in the expanded panel of vemurafenib-resistant melanoma cell lines

\begin{tabular}{|c|c|c|c|c|c|}
\hline Cell line & $\begin{array}{c}\text { BRAF } \\
\text { inhibitor }\end{array}$ & $\begin{array}{l}\text { Tubulin } \\
\text { inhibitor }\end{array}$ & CI at $E_{50}$ & CI at $\mathrm{ED}_{75}$ & CI at $\mathrm{ED}_{90}$ \\
\hline \multirow{6}{*}{$\begin{array}{l}\text { MDA-MB- } \\
435 \text { VemR }\end{array}$} & \multirow[t]{3}{*}{ Vemurafenib } & ABI-274 & 0.59 & 0.55 & 0.52 \\
\hline & & Docetaxel & 0.55 & 0.94 & 1.03 \\
\hline & & Vinblastine & 0.79 & 0.95 & 1.13 \\
\hline & \multirow[t]{3}{*}{ Dabrafenib } & ABI-274 & 0.37 & 0.43 & 0.51 \\
\hline & & Docetaxel & 0.84 & 0.84 & 0.85 \\
\hline & & Vinblastine & 0.44 & 0.44 & 0.47 \\
\hline \multirow[t]{3}{*}{ 451LuMR } & \multirow[t]{3}{*}{ Vemurafenib } & ABI-274 & 0.96 & 0.74 & 0.68 \\
\hline & & Docetaxel & 0.44 & 0.60 & 0.82 \\
\hline & & Trametinib & 0.73 & 1.15 & 1.03 \\
\hline \multirow{3}{*}{$\begin{array}{l}\text { WM983B } \\
\text { BR }\end{array}$} & \multirow[t]{3}{*}{ Vemurafenib } & ABI-274 & 0.29 & 0.39 & 0.74 \\
\hline & & Docetaxel & 0.24 & 0.49 & 1.04 \\
\hline & & Trametinib & 2.03 & 1.26 & 0.94 \\
\hline
\end{tabular}

The combination index (CI) values were calculated based on the results from cell viability MTS assay $(n=5)$. CI $<0.9$ indicates synergism; $0.9 \leq \mathrm{CI} \leq 1.1$ indicates additive effect; $\mathrm{CI}>1.1$ indicates antagonism between the two tested drugs. 
eliminate or reduce melanoma tumor resistances to BRAF or MEK1/2 inhibitors $(28,37$, $39,80,124,133,134)$. Inhibitors to the $\mathrm{RAF} / \mathrm{MEK} / \mathrm{ERK}$ pathway mainly produce $\mathrm{G}_{1}$ cell-cycle arrest rather than melanoma tumor cell death. Thus a combination of agents targeting different components in the same pathway (e.g. combination of vemurafenib and MEK1/2 inhibitors), while effective initially (109), may not maintain long-lasting synergy against resistant cells that can escape from these $G_{1}$ cell-cycle arrests.

Activation of the PI3K/AKT/mTOR signaling pathway has been shown to contribute to the diminished sensitivity to ERK1/2 inhibition in human melanoma cell lines (135), and several recent studies have clearly demonstrated the synergistic combination of an inhibitor targeting PI3K/AKT/mTOR pathway and a BRAF inhibitor or a MEK inhibitor $(35,39,135,136)$. Recently several novel classes of compounds were reported as inhibitors of tubulin polymerization and also showed strong inhibition of the AKT pathway (137-139). In addition, constitutively active PI3K/AKT pathway has been shown to lead to multidrug resistances to microtubule-targeted tubulin-polymerizing agents (MTPA) and inhibition of PI3K/AKT-mediated signaling pathway has been shown to sensitize cancer cells to MTPA-induced apoptosis (140). These studies indicate a close interplay between tubulin polymerization inhibitors and AKT down regulation in cancer cells. In addition, Nikolas K.H et al. has recently reported that MEK inhibitor AZD6244 induced growth arrest in melanoma cells and tumor regression when combined with docetaxel (141). Interestingly, our current report is consistent with these studies. The in vivo studies presented here show an effective combination treatment in tumor cells that are already vemurafenib-resistant by using A375RF21 xenograft models. It is conceivable that if we used the combination before tumor cells became resistant to vemurafenib, tumor regression may be more enhanced and we could significantly delay or even prevent the development of resistant tumor cells. This could translate into at least a substantially longer progression-free time in patients, and/or enhanced disease regression. Collectively, our study offers the first direct evidence and a rationale for combining a potent tubulin inhibitor with an inhibitor targeting the RAF/MEK/ERK pathway for greatly improved therapy for melanoma patients. 


\section{CHAPTER 3. LITERATURE REVIEW OF SURVIVIN AND ITS ANTAGONISTS}

\subsection{The Structure and Tissue Distribution of Survivin}

Survivin (encoded by BIRC5), the smallest member of the inhibitor of apoptosis proteins (IAPs), is a $16.5 \mathrm{kDa}$ protein that consists of an $\mathrm{N}$-terminal $\mathrm{Zn}^{2+}$-binding baculoviral inhibitor-of-apoptosis (IAP) repeat (BIR) domain and an amphipathic Cterminal helix (142-144). Two molecules of human survivin form a homodimer through a hydrophobic interface, which locates in a region between the BIR domain and C-terminal alpha helix (145).

Survivin is ubiquitously expressed in lung, breast, gastric, bladder, colon and prostate cancer, lymphoma, esophageal carcinoma and osteosarcoma cells but undetectable in finally differentiated adult normal tissues (146-149). And its tumor specific distribution pattern in human tissues has distinguished survivin from other members in IAP family $(150,151)$. Overexpression of survivin is positively correlated with tumor invasion, multiple drug resistance, and unfavorable prognosis with decreased patient survival rates $(150,152-156)$. Recent studies also revealed that there is a definite correlation between high levels of survivin and the over-activation of known oncogenic pathways (HIF-1 $\alpha$ (157), HSP90 $(158,159)$ PI3K/AKT $(160,161)$, ERK (162), Bcl-2 $(14,15)$ and RAS pathways $(163,164))$, but negatively correlate with tumor suppressor genes(165-167) (p53, PTEN). In addition, the presences of survivin in physiological fluids including urine, bronchial aspirate, pleural effusion and plasma make survivin as a useful biomarker to non-invasively monitor tumor relapse and metastasis $(168,169)$.

\subsection{The Expression and Regulation of Survivin}

Survivin expression dominantly peak in the $\mathrm{G}_{2} / \mathrm{M}$ phase, followed by a rapid degradation via the ubiquitin-proteasome pathway during $G_{1}$ phase $(161,170)$. Studies have shown that this cell-cycle dependent pattern results from the reduced degradation of survivin rather than the up-regulated gene transcription in $\mathrm{G}_{2} / \mathrm{M}$ phase (171). The halflife of survivin is as short as $30 \mathrm{~min}$ and its degradation is considered to be involved with the X-linked inhibitor of apoptosis (XIAP) - associated factor 1 (XAF1). The XAF1XIAP interaction activates the ubiquitin ligase activity of XIAP and thus degrades survivin (172). Meanwhile, heat shock protein 90 (Hsp90), one of the most important molecular chaperones in mammalian cells, binds to survivin BIR domain through ATPase domain of Hsp90 and protect survivin from the ubiquitin-mediated degradation (Figure 3-1)(158). 


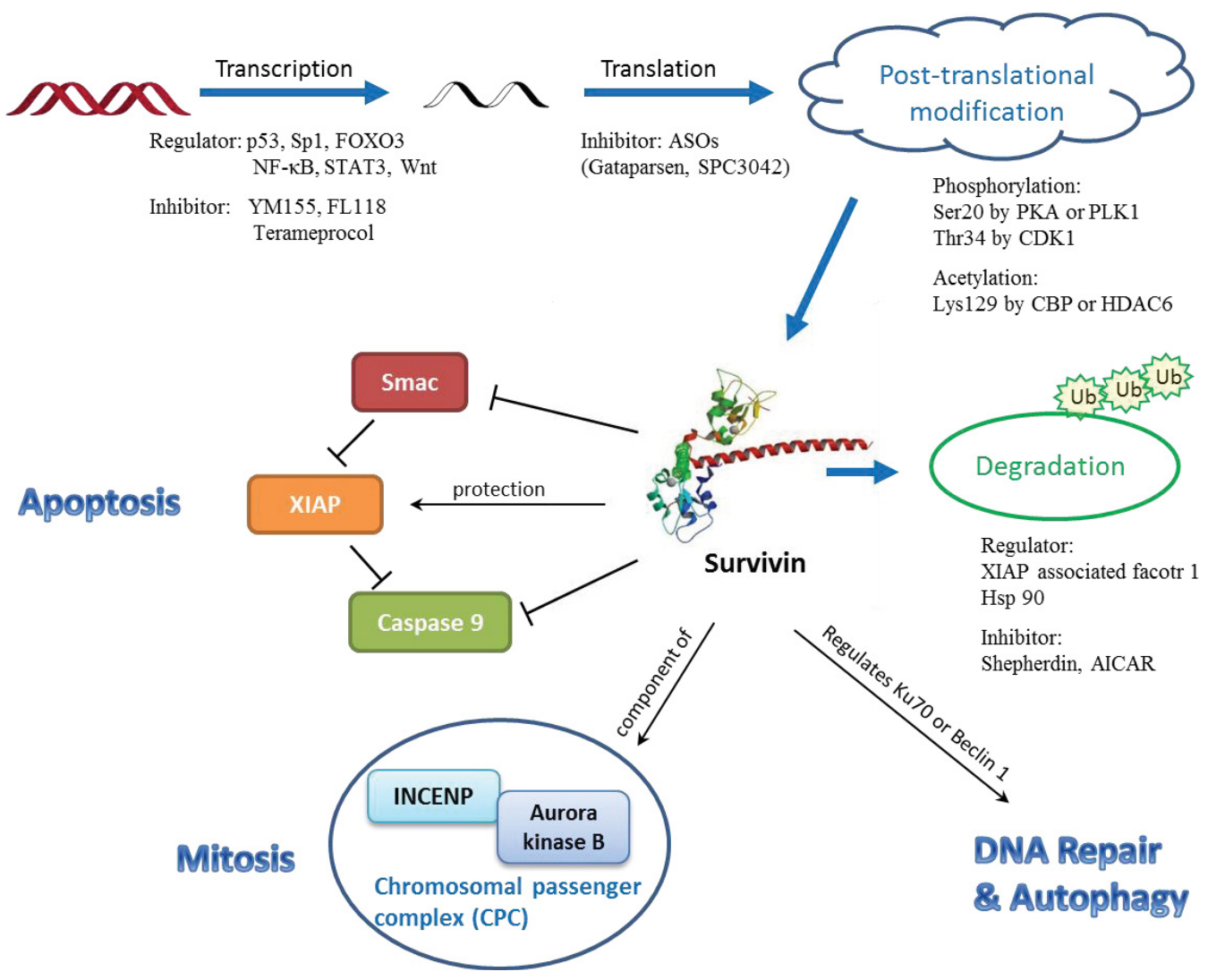

Figure 3-1. The nodal functions of survivin and targets of survivin inhibitors 


\subsubsection{Control of Survivin Gene Expression by Transcription Factors}

Multiple upstream factors control the gene expression of survivin, including but not limiting to tumor suppressor p53, specificity protein 1 (Sp1), and Forkhead box O3 (FOXO3).

The interplay between p53 and survivin is mutual. In one hand, p53 can direct bind to and stimulate the DNA methyl-transferase (DNMT) 1(173), which methylates survivin promoter and leads to survivin gene repression. On the other hand, ectopic survivin decreases the mRNA level of p53 and Mdm2 in Adriamycin-treated MCF7 cells (174). In many cases, loss of wild-type p53 and survivin overexpression co-exist in cancer with elevated resistance to therapy. Also $p 53$ gene mutant status is found to be highly associated with the expression of survivin and its anti-apoptotic splice variants, survivin-deltaEx3 and survivin-3B, in 163 breast carcinoma tissues (175).

Chen et al (176) has shown that Sp1 can cooperate with Sp3 to regulate the basal expression level of survivin in Hela cells. Sp1 directly bind to the TATA-less regions of survivin promoter through the interaction with two $\mathrm{Sp} 1$ binding sites on that promoter (position -148 to -153; position -127 to -140). Furthermore, Sp1 is co-overexpressed with survivin in adenocarcinoma of lung cells A549 (177). In this model, Sp1 up-regulates survivin expression through direct binding with survivin promoter and lead to the deduction of caspase-9 activity (177).

FOXO3 increases in cell response to high level of intracellular reactive oxygen species (ROS). The nuclear translocation of FOXO3 and activation of FOXO3/FKHRL1 represses the survivin transcription in human neuroblastoma (NB) tumor and sensitize it to doxorubicin treatment (178). In fact, survivin induces mitochondria fragment, reduces mitochondrial respiration and thus protects NB cells from the FOXO3-mediated mitochondrial apoptosis (179).

Other nuclear factors like kappa-light-chain-enhancer of activated B cells (NF$\kappa B)(180)$, signal transducer and activator of transcription-3 (STAT3) (181), Winglesstype MTV integration site family member (Wnt) (182) have been reported to increase the mRNA level of survivin. And insulin-like growth factor (IGF) can promote the translation of survivin and prostate cancer cell survival through activating phosphoinositide 3-kinase (PI3K)/ AKT/ mammalian target of rapamycin (mTOR) signaling pathway (183).

\subsubsection{Post-translational Modification of Survivin}

Besides the mRNA-level regulation of survivin, post-translational modification determines the survivin function and its distribution among nuclear, cytoplasm and mitochondria. Generally, two major types of post-translational modification survivin are reported in the literature: phosphorylation and acetylation (Figure 3-1). 
Survivin can be phosphorylated at several sites that shape its molecular functions, including Thr34, 53 and 117 plus Ser20. Thr34 of survivin is phosphorylated by cyclin

dependent kinase $(\mathrm{CDK}) / \mathrm{p} 34^{\text {cdc2 }}$-cyclcin B1 and this process results in "activated" survivin that is able to bind with caspase-9 in Hela cells (184). Ser20 of survivin can be phosphorylated by protein kinase A (PKA) or polo-like kinase 1 (PLK1) but evidences exist that they contribute to different molecular function of survivin. The phosphorylation of survivin through PKA-mediated mechanism specifically occurs in cytosol rather than mitochondria and leads to the stabilization of XIAP and subsequently anti-apoptosis effect (185). Interestingly, in cytosol PLK1-mediated phosphorylation of survivin at Ser20 is discovered to activate Aurora B and promote the formation of chromosomal passenger complex (CPC) in nucleus. In this way, PLK1 regulates cell mitosis (186).

Next, the acetylation of survivin at residue Lys 129 is regulated by factors like CREB binding protein (CBP) and histone deacetylase 6 (HDAC6). CBP directly acetylates survivin and catalyzes its homo-dimerization and accumulation in nucleus. Subsequently, the acetylated survivin binds to the N-terminal of STAT3 and decreases STAT3 oncogene activity (187). HDAC6 de-acetylates survivin and promotes the nuclear export of survivin in MCF7 breast cancer cells (188).

\subsection{The Nodal Functions of Survivin}

Functionally, nuclear survivin is a component of CPC (189), which regulates chromosomal segregation during cell division (190). Cytoplasmic and mitochondrial survivin inhibits the cell apoptosis both directly and indirectly through caspases-mediated mechanisms (Figure 3-1) (150, 154, 155).

\subsubsection{Apoptosis}

Survivin interferes the apoptosis process in both caspases-dependent and caspases-independent mechanisms. As indicated above, Thr34 phosphorylated survivin in mitochondria can bind directly with caspase- 9 and thus prevent caspase- 9 activation. Also the formation of survivin-XIAP complex protects ubiquitin-mediated degradation of XIAP and thus indirectly inhibits caspase-9 activation (172). Interestingly, survivin is found to bind with caspase-3 and caspase-7 with high affinity in vitro under cell-free condition (191). Meanwhile, the anti-apoptotic effect of survivin relies on the direct interaction between survivin and a mitochondria protein, Smac/DIABLO (second mitochondria-derived activator of caspases). In this case, survivin sequesters Smac/DIABLO away from XIAP binding and inhibits Smac/DIABLO releasing from mitochondria $(144,192,193)$. Structure analysis showed that Smac/DIAOBLO competitively binds with survivin BIR domain through N-terminal Ala-Val-Pro-Ile (AVPI) tetra-peptide motif like it does with other IAP proteins $(144,194)$. Additionally, survivin induces the translocation of mitochondrial apoptosis-inducing-factor (AIF) from cytoplasm to nucleus and promote caspase-independent pathway with DNAfragmentation (195). 


\subsubsection{Mitotic}

CPC consists of two distinct units: an enzymatic complex contains Aurora B and $\mathrm{C}$-terminal fragment of the inner centromere protein (INCENP), and a chromosomal localization complex contains N-terminal fragment of INCENP, survivin and Borealin (196). BIR domain of survivin interacts with phosphorylated $\mathrm{Thr} 3$ on histone $\mathrm{H} 3$ (H3T3ph) through N-terminal Ala1 (194) and activates the kinase function of Aurora B (197). The formation of CPC and recognition of survivin BIR domain with Aurora B are crucial for both the proper chromosomal alignment and mitotic spindle assembling (189).

\subsubsection{DNA Repair and Autophagy}

The mechanism of survivin interfering with DNA-repair and autophagy has not been studied as clear as its function involves apoptosis and cell division. Current evidences show that nucleus survivin interacts with or regulates the level of various DNA-damage/repair-associated modules like Ku70 (a DNA-damage molecular sensor) (198) and DNA-dependent protein kinase catalytic subunit (DNA-PKCs) (199). Silencing survivin may induce autophagy-dependent apoptosis in hepatocellular carcinoma cells (200). And the down-regulation of survivin through knock-out of Beclin 1, an important autophagy regulator, sensitizes glioma cells to TRAIL-induced apoptosis (201).

\subsection{Current Antagonists of Survivin as Anti-tumor Agents}

\subsubsection{Small Molecule Inhibitors}

Survivin is not enzymatic protein and doesn't occupy common "druggable" binding site on its surface. Therefore, it makes the discovery of small molecule survivin antagonist fairly difficult. Generally, small molecule agents under current development antagonize survivin function in three distinct mechanisms.

The first category is survivin expression modulator, which blocks either survivin gene promoter or transcriptional factor. For example, YM155, which is under Phase II clinical investigation, selectively suppresses the gene expression of survivin over the other IAPs and induces caspase or autophagy-mediated apoptosis in various cancer type (202-206). In xenograft models, YM155 can lead to tumor regression and sensitize the tumor to the treatment of commonly used chemotherapy agents $(206,207)$. Another example is FL118, which binds to survivin gene promoter region at low nano-molar concentration in a $\mathrm{p} 53$-independent manner and shows great anti-tumor efficacy in xenograft models $(208,209)$. Terameprocol (EM1421) inhibits Sp1 and subsequently reduces transcription of survivin, CDK1 and $\operatorname{VEGF(210).~Its~administration~as~vaginal~}$ ointment is well tolerated in Phasel/II trials in women with HPV-linked cervical squamous intraepithelial neoplasia but limited systemic absorption (211). However, in a more recent Phase I study, where terameprocol is co-currently administered with enzyme- 
inducing antiseizure drugs (EIASDs) intravenously for 5 consecutive days each month in high-grade glioma, the combination therapy is well tolerated without alteration of terameprocol pharmacokinetic profile and myelosuppression side effects (212).

The second type of small molecule survivin inhibitor interferes with the interaction between survivin and other proteins like Hsp90 and Smac/DIABLO. A peptidomimetic of survivin sequence K79 to L87, shepherdin, can bind to ATP pocket on Hsp90 N-terminus and then destabilize survivin together with other Hsp90 client proteins like AKT, CDK6 and telomerase $(213,214)$. Shepherdin induces both apoptotic and nonapoptotic massive cancer cell death and is well tolerated in mice model. The non-peptidic 5-aminoimidazole-4-carboxamide-1-b-D-ribofuranoside (AICAR) directly binds to chaperone through hydrogen bonds between ribose ring of AICAR and Asp93 of Hsp90 protein. And it destabilizes survivin and exerts anti-cancer activity through mechanisms similar to shepherdin (215). Oikawa et al. has reported the identification of a 5-dezaflavin analog that selectively inhibits the interaction of survivin-Smac/DIABLO but not survivin-INCENP through high through-put system. This compound sensitizes the lung carcinoma A549 cells to doxorubicin-induced DNA damage and synergistically enhances the apoptotic cell death (193).

Some other small molecule survivin inhibitors regulate the post-translational modification of survivin mainly through the inhibition of CDK-mediated phosphorylation of survivin Thr34. Reported compounds in this category include flavopiridol (216), NU6140(217) and purvalanol A $(218,219)$. But usually their anti-cancer effect is not solely based on the inhibition of survivin phosphorylation. Among them, flavopiridol, as a flavonoid alkaloid pan-CDK inhibitor and the first CDK inhibitor to enter the clinic was approved as an orphan drug for treatment of chronic lymphocytic leukemia and is currently undergoing phase II studies as monotherapy and also as in combination regimes with traditional chemotherapy agents $(220,221)$.

\subsubsection{Survivin Antisense Oligonucleotides (ASOs)}

ASOs are developed as to specifically hybridize with complimentary survivin mRNA and lead to destruction of survivin mRNA by RNAse H (222). Gataparsen (or LY2181308) is the most advanced survivin ASO and it has an 18-mer 2'-Omethoxyethyl-(MOE) modified structure, which protects it from the nuclease degradation. Upon binding to translational initiation condo of survivin, gataparsen activates caspase-3 apoptosis cascade in tumor cells without affecting other proteins and it is in Phase II clinical study. The maximum tolerable dose of gataparsen is determined as $750 \mathrm{mg}$ in patients with various solid malignancies and its accumulation in tumor tissue and survivin-deregulation effect is confirmed in a Phase I study (223). However, data revealed from a Phase II study in patients with castration-resistant prostate cancer (CRPC) shows that the combination therapy using gataparsen together with standard treatment docetaxel/prednisone doesn't achieve better efficacy compared to a control group receiving only standard docetaxel/prednisone (224). 
Another survivin ASO under development is SPC3042 (or EZN-3042), which is a 16-mer locked nucleic acid ASO that binds to exon 4 of the survivin transcript (225). In lung carcinoma A549 and Calu-6 xenograft models, SPC3042 induces 60\% downregulation of survivin mRNA and 37-45\% tumor growth inhibition (TGI) as single treatment and $83 \%$ TGI as combination treatment with paclitaxel (226). However, recent data from a Phase I study has reported dose-limiting grade 3 toxicity of SPC3042 as single treatment in children with relapsed acute lymphoblastic leukemia (ALL), although 2 out of 5 patients are detected with decreased survivin expression. And the study was terminated due to the severe side effect without further clinical development (227).

\subsubsection{Dominant Negative Survivin (DNS)}

DNS is a single amino acid mutant form of survivin protein. DNS is designed to form heterodimer with wild-type survivin and then interfere with the survivin biological function. Study has shown that delivering DNS plasmid DNA or DNS protein into cancer cell in vitro or tumor tissue in vivo can induce wide-spread apoptosis and tumor growth inhibition. One of the well-studied DNSs is the T34A survivin, of which Thr34 is replaced with alanine. T34A DNS is also referred as "phosphorylation-dead mutation survivin", since the phosphorylation of survivin by CDK/p34 ${ }^{\text {cdc2 }}$-cyclcin B1 on Thr34 is critical for its stability and anti-apoptotic function. T34A survivin triggers mitochondria apoptosis pathway, promotes p53-associated apoptosis and enhances the anti-cancer efficacy of chemotherapy agents like cisplatin and doxorubicin (228). Transfection of T34A survivin plasmid into YUSAC2 melanoma cells prevented tumor formation upon s.c. injection in mice model and induction of T34A in established melanoma tumors leads to $60-70 \%$ tumor growth inhibition (229). Fusing an HIV-derived TAT peptide sequence into T34A survivin greatly increases the cell permeability and protein transduction efficiency. The TAT-T34A survivin enters YUSAC2 or WM793 melanoma cells within 30 min and leads to caspase-3 activation and cell detachment. Intraperitoneal (i.p.) injection of TAT-T34A survivin results in rapid tumor accumulation in $1 \mathrm{hr}$ with induction of apoptosis and aberrant nuclei formation (230).

Besides, the replacement of Cys84 on wild-type survivin with alanine generates C84A survivin and disrupts the zinc chelating of BIR domain on survivin then abolishes the anti-apoptosis effect of survivin protein. When C84A survivin coded plasmid is intratumorally injected together with a costimulatory of T cells, B7-1, mouse EL-4 thymic lymphoma tumor growth has been significantly inhibited. And the combination treatment generates higher antitumor cytotoxic T-lymphocyte (CTL) activity comparing with the single treatment groups (231). When survivin C84A mutant is fused with a $9 \mathrm{~N}$-terminal arginine residues (R9, cell-permeable peptide carrier), the formed SurR9-C84A protein shows dual actions. In normal cells, it protects differentiated SK-N-SH human neuroblastoma cells from activated T-cell neurotoxicity (232) and increases the proliferation of differentiated SK-N-SH and HCN-2 neurons (233). In cancerous cells, it sensitizes prostate cancer cells to TNF- $\alpha$ and induces tumor-specific intrinsic and extrinsic apoptosis in colon cancer cells (234). Interestingly, researchers have studied that the adenovirus transduction of double point mutant survivin (TC34,84AA) into 
hepatocellular cancer cells exerts stronger cytotoxicity effect and this form of survivin mutant is more sensitive to the ubiquitin-mediated degradation (235).

\subsection{Conclusion}

Despite extensive efforts since the discovery of survivin in 1997, only a few survivin antagonists are available for clinical test. Given the fact that survivin is preferentially distributed in cancer cells rather than normal tissues, survivin antagonists usually are well-tolerated and inherently show low toxicities $(153,155,202)$. However, inadequate anti-tumor efficacy leads to the major failure in clinical trials of survivin antagonists. Two categories of factors contribute to the observed efficacy limits. On one hand, the degradation and unspecific delivery of biological agents include antisense oligonucleotides (e.g., LY2181308 and SPC3042) (225, 236, 237), ribozymes $(29,30)$ and small interfering RNAs $(238,239)$ restricted their internalization into tumor cells. On the other hand, the high degree of co-overexpression of survivin with drug-resistant markers like $\mathrm{ABC}$ transporter proteins hindered the efficacy of some survivin antagonists $(153,240)$. For example, the tumor cell uptake of YM155, the most potent survivin gene promoter inhibitor $(203,205,207)$, is impeded by P-glycoprotein (Pgp) drug efflux pump (241), which is practically co-overexpressed with survivin in drug-resistant breast cancer, renal cell carcinoma and acute myeloid leukemia patients (242-244). Therefore, the development of new survivin inhibitors that can effectively overcome multidrug resistance with good pharmacokinetic properties is highly needed. 


\section{CHAPTER 4. DISCOVERY OF NOVEL SMAC MIMETIC AS SELECTIVE SURVIVIN INHIBITOR*}

\subsection{Introduction}

The family of inhibitor of apoptosis proteins (IAP) bind to caspases, block the assembling of pro-apoptotic protein signaling complexes, and thereby prevent the activation of caspase proteolytic cleavages and the subsequent triggering of apoptosis. There are eight IAP proteins identified in human: NAIP, cIAP1, cIAP2, XIAP, ML-IAP, ILP2, survivin, and apollon $(172,245,246)$. As overexpression of IAPs frequently occurs in cancer cells and has been linked to tumor progression, treatment failure, and poor prognosis, IAPs are considered to be promising therapeutic targets in either directly eliciting cell death or lowering the threshold for cell death induction of current anticancer therapeutics $(245,247,248)$.

Smac/DIABLO (second mitochondria-derived activator of caspase/direct IAP binding protein with low $\mathrm{pI}$ ) molecules released from mitochondria antagonize the IAPs and can protect caspases from IAP inhibition $(245,247-250)$. Smac interacts with IAPs mainly via its N-terminal AVPI binding motif. A proven strategy for inhibiting IAPs is to disrupt the interaction between an IAP and Smac by developing Smac mimetics (249). Smac-mimicking IAP antagonists can induce apoptosis in tumor cells and effectively inhibit tumor growth in mice (251-256). They can also inactivate nuclear factor $\kappa \mathrm{B}(\mathrm{NF}-$ $\kappa \mathrm{B})$ and produce secretion of tumor necrosis factor $\alpha(\mathrm{TNF}-\alpha)(248,255)$. Several Smac mimetics have entered late stage preclinical development or human clinical testing as novel cancer therapeutics (251-256). For example, Birinapant (TL32711), now in Phase II study, can effectively suppress cIAP1 and XIAP at well-tolerated doses and promises antitumor activity either as a single agent or in combination with standard-of-care chemotherapeutic drugs in adult patients with advanced solid tumors or lymphoma(257). However, no IAP inhibitors have been approved by the FDA as of today, and there are limitations with many existing IAP inhibitors. For example, YM155 is a well-known survivin inhibitor that has gone through clinical trials, but it has been shown to be a substrate for the P-glycoprotein (Pgp) drug efflux pump (241), suggesting that it could suffer from multidrug resistance (MDR) in its eventual clinical use. Thus, exploring novel scaffolds to develop potent and selective IAP antagonists is still much needed. Since all IAP proteins share the signature baculoviral IAP repeat (BIR) domain (245), which interacts with Smac, shape-based virtual screening will be helpful in identifying potential small-molecule Smac mimetics for regulating apoptosis in cancer cells.

In this chapter, we describe our efforts to identify novel small-molecule Smac mimetics through an integrated virtual screening and biological validation approach.

* Modified with permission. Wang J, Li W. Discovery of novel second mitochondriaderived activator of caspase mimetics as selective inhibitor of apoptosis protein inhibitors. J Pharmacol Exp Ther. 2014;349: 319-329. 
Their efficiency in inhibiting IAPs, especially XIAP and survivin (BIRC5), and inducing apoptosis in cancer cells was further validated in serial biological studies both in vitro and in vivo. These compounds represent novel scaffolds for IAP inhibition and can be further optimized to serve as a potential targeted agent for various types of cancers.

\subsection{Methods}

\subsubsection{Shape-based Virtual Screening}

The University of Cincinnati's Drug Discovery Center (UC DDC) Library (contains 362,910 compounds) was used to conduct the shape-based virtual screening. All structures were first prepared using the LigPrep module in Maestro Suite 2012 (Schrodinger, LLC, New York, NY) to generate conformers and charged states. We used the phase_shape program in Canvas (version1.4, Schrodinger, LLC, New York, NY). Conformers with a shape similarity below 0.7 were filtered out, and hits with a similarity value above this threshold were selected for subsequent molecular docking process.

\subsubsection{Molecule Docking}

Crystal structures of Smac bound to XIAP BIR3 domain (PDB ID: 1G73 (249) and 1TW6 (258)) were processed with the Protein Preparation Wizard, and the grid of AVPI binding site was defined by Glide (version 5.7, Schrodinger, LLC, New York, NY). 1000 hits with top-ranked similarity value were docked into the AVPI binding site in each separate complex. The best docking complexes were subject to restricted molecular dynamics to release any strains by using the Macromodel module with OPLS2005 force field. The ligand and its surrounding residues within $15 \AA$ \& were allowed to move freely, while residues outside the 15 - $\AA$ radius were kept rigid.

\subsubsection{Cell Culture and Reagents}

Human melanoma A375 cell line and human prostate PC-3 and DU145 cell lines were acquired from American Type Culture Collection (Manassas, VA). Human melanoma M14 cell line was kindly provided by Dr. Robert Clarke (Georgetown University, Washington, DC). Human keratinocyte Hacat cell line was a gift from Dr. Andrzej T. Slominski (University of Tennessee Health Science Center, Memphis, TN). Human dermal fibroblast adult cells (HDFa) cells were purchased from Life Technologies (Thermo Fisher Scientific Inc., Waltham, MA). All cell lines were authenticated prior to use for this study. Cancer cells were cultured in DMEM (for melanoma cells) or RPMI 1640 (for prostate cancer cells) medium (Mediatech, Inc., Manassas, VA), supplemented by $10 \%$ fetal bovine serum (FBS, Atlanta Biologicals, Lawrenceville, GA), $1 \%$ antibiotic/antimycotic mixture (Sigma-Aldrich, St. Louis, MO) and $5 \mu \mathrm{g} / \mathrm{mL}$ bovine insulin (Sigma-Aldrich, St. Louis, MO). Hacat cells were cultured in 
10\% FBS supplemented DMEM medium. HDFa cells were maintained in supplemented Gibco ${ }^{\circledR}$ Medium 106 (M-106-500, Life Technologies). Compounds were dissolved in dimethyl sulfoxide (DMSO, Sigma-Aldrich, and St. Louis, MO) to make a stock solution of $10 \mathrm{mM}$. Compound solutions were freshly prepared by diluting stocks with cell culture medium before use (final solution contained less than 0.5\% DMSO). Proteasome inhibitor MG-132 was purchased from Enzo Life Sciences, Inc. (Farmingdale, NY).

\subsubsection{Cell Viability Assay}

Resources of cell line and culture reagent can be found in Supplemental Information Method. The anti-proliferation effect against cancer cells of all UC compounds was first screened in vitro by using the MTS assay as described previously $(127,129,259)$. Then the $\mathrm{IC}_{50}$ values of selected hits were tested through the sulforhodamine B (SRB) assay as reported before (129).

\subsection{5 siRNA Silencing Survivin or XIAP Expression}

SignalsSilence ${ }^{\circledR}$ survivin or XIAP siRNA and fluorescein conjugated control siRNA were purchased from Cell Signaling Technology, Inc. (\#6351, \#6446, \#6201, Danvers, MA). They were transfected into pre-seeded A375 or PC-3 cells by mixing with Lipofectamin ${ }^{\circledR}$ RNAiMAX reagent (Invitrogen, Thermo Fisher Scientific Inc., Waltham, MA) at the final concentration of $100 \mathrm{nM}$ in serum-free medium. $24 \mathrm{hr}$ after the transfection, cells were treated with compound solution to determine the changes of cell viability.

\subsubsection{Caspase Functional Assay}

The caspase activity of cancer cells treated by DMSO control or compound solution after 24-h or 48-h incubation was analyzed using Caspase-Glo ${ }^{\circledR} 3 / 7$ and CaspaseGlo ${ }^{\circledR} 9$ assay kits from Promega Corporation (Madison, WI) as per manufacturer's instructions. The readings of relative luminescence unit (RLU) were normalized by the cell viability results from the same well determined by compatible CytoTox-Flour ${ }^{\mathrm{TM}}$ Cytotoxicity assay kit (Promega, Madison, WI).

\subsubsection{Western Blotting}

After treatment for the indicated time, A375, PC-3, and DU145 cells were lysed to determine by Western blotting the relevant IAP family protein levels. Primary rabbit antibodies were purchased from Cell Signaling Technology, Inc. (Danvers, MA): anticIAP1 (\#7065), anti-cIAP2 (\#3130), anti-survivin (\#2808), anti-XIAP (\#2045), anti-livin (\#2978), or anti-GAPDH (\#3683). Then anti-rabbit IgG HRP-conjugated secondary antibody (Cell Signaling, \#7071) was used to detect the target protein level Lane 
intensities were quantified with ImageJ software (US National Institutes of Health, Bethesda, MD).

\subsubsection{Flow Cytometry Analysis}

Flow cytometry analysis was performed as described previously (129). Briefly, A375 cells $(n=3)$ were synchronized through $24 \mathrm{~h}$ starvation in growth media containing only $0.1 \%$ FBS. Then the cells were treated with 0,1 , or $4 \mu \mathrm{M} \mathrm{UC}-112$ in full growth media $(10 \% \mathrm{FBS})$ for another $24 \mathrm{~h}$. Then the cell-phase distribution was determined on a BD LSR-II cytometer (BD Biosciences, San Jose, CA) with 10,000 cells scored, and data were processed using Modfit 2.0 program (Verity Software House, Topsham, ME).

\subsubsection{Human Melanoma A375 Tumor Xenograft Model and Treatment}

We first estimated the acute maximum tolerable dose (MTD) for compound UC112. Progressively increasing injection doses via intra-peritoneal (i.p.) injection route to BXD mice $(n=3)$ determined the estimated MTD to be above $200 \mathrm{mg} / \mathrm{kg}$ with one-week continuous treatment. To ensure a large safety margin during the three-week treatment and considering the practical doses in clinical, we scaled down the dose to $20 \mathrm{mg} / \mathrm{kg}$ and $40 \mathrm{mg} / \mathrm{kg}$ in the xenograft model study.

Seven- to 8-week-old male nude mice were purchased from Charles River Laboratories International, Inc. (Wilmington, MA). Right before use, A375 cells were suspended in ice-cold phenol red-free and FBS-free DMEM medium and mixed with high concentration Matrigel (BD Biosciences, San Jose, CA) at a ratio of 1:1.100 $\mu \mathrm{L}$ of this mixture containing $3 \times 10^{6}$ A375 cells was injected subcutaneously to the left-side dorsal flank of each mouse. One week after the inoculation, the mice were randomized into four groups $(n=7)$, and treatments started. UC-112 compound was suspended in sterile PBS buffer and administered through intraperitoneally (i.p.) injection once per day, 5 days per week, for 3 continuous weeks. Vehicle control group was i.p. injected with the same volume $(100 \mu \mathrm{L})$ of PBS buffer at the same dosing frequency. At the end of the experiments, mice were euthanized, and tumor tissues were isolated and weighed separately. Then one small piece (around $50 \mathrm{mg}$ ) from each tumor was cut and stored in liquid nitrogen immediately. The rest of the tumor tissue was fixed in $10 \%$ buffered formalin phosphate solution for more than 1 week before pathology staining analysis.

Tumor volume and body weight of each mouse were evaluated 3 times a week. We calculated the tumor volume with the formula $a \times b^{2} \times 0.5$, where $a$ and $b$ represented the larger and smaller tumor diameters. Data were presented as mean $\pm \mathrm{SD}$ for each group and plotted as a function of time. Tumor growth inhibition (TGI) ratio (\%) was calculated as $100-100 \times\left[\left(T-T_{0}\right) /\left(C-C_{0}\right)\right]$, where $T, T_{0}, C$, and $C_{0}$ were the mean tumor volume for the specific group on the last day of treatment, mean tumor volume of the same group on the first day of treatment, mean tumor volume for the vehicle control 
group on the last day of treatment, and mean tumor volume for the vehicle control group on the first day of treatment, respectively.

\subsubsection{Pathology Analysis}

Tumor tissues fixed in formalin buffer for more than 1 week were stained with hematoxylin and eosin. All the slides were scanned to create a digital replica of the entire tissue on a glass microscopic slide by using the ScanScopeXT at $0.25 \mathrm{pixel} / \mu \mathrm{m}$.

\subsubsection{TUNEL Assay}

To evaluate the nuclear DNA fragmentation within the A375 tumor tissue, Terminal deoxynucleotidyltranferase (TdT)-mediated dUTP nick end labeling (TUNEL) assay was performed using the DeadEndTM Fluorometric TUNEL system (Promega, Madison, WI) following the manufacturer's protocols. Briefly, the formalin-fixed and paraffin-embedded tumor sections were deparaffinized and rehydrated before $100 \mu \mathrm{L}$ of $20 \mu \mathrm{g} / \mathrm{mL}$ proteinase $\mathrm{K}$ solution was added to premeabilize the tumor sections. After preequilibration in room temperature for 10 minutes, tumor sections were incubated with nucleotide mix and $\mathrm{rTdT}$ enzyme at $37^{\circ} \mathrm{C}$ for one hour. The reaction was stopped with saline-sodium citrate (SSC) buffer and the tumor sections were washed with PBS to remove the excess reagents. These slides were mounted in VECTASHIELD ${ }^{\circledR}+$ DAPI $(H-$ 1500, Vector Labs, Burlingame, CA) to stain nuclei. Finally, coverslips were added and the slides were analyzed immediately under a fluorescence microscope (EVOS ${ }^{\circledR}$ FL Cell Imaging System, Thermo Fisher Scientific Inc., NY).

\subsubsection{Statistical Analysis}

Data were analyzed using Prism Software 5.0 (GraphPad Software, Inc., San Diego, CA). The statistical significance ( $P$ value) was evaluated by one-way ANOVA followed by nonparametric Dunnett's test for in vitro apoptosis detection and in vivo xenograft study. Every treated group was compared to the vehicle group separately.

\subsection{Results and Discussion}

\subsubsection{Identification of Initial Hits from Virtual Screening}

We started the study with shape-based virtual screening using the phase shape program in Canvas. It initiates finding trial alignments based on the principle of the distribution of atom triplets, then refines the top alignments to maximize the volume overlap(260). The 3D structure of Smac N-terminus AVPI tetrapeptide (PDB ID: 1G73 (249)) in its bioactive conformation was selected as the query template to screen 
molecules within the prepared compound library that could achieve similar conformations to that of the active AVPI peptide. In our study, the highest shapesimilarity score of UC library compounds reached 0.81 ; over 800 compounds had scores over 0.75 . Figure 4-1 illustrates the alignments generated from the similarity screening where template ligand AVPI peptide overlapped well with representative hit compounds UC-222 and UC-112.

To validate the theoretical interaction between the shape-based screening hits and IAP protein, we docked the top 1000 hits into the Smac AVPI binding pocket in two widely used crystal complexes of Smac-XIAP (X chromosome-linked IAP) BIR3 domain (PDB ID:1G73 (249) and 1TW6 (258)) . We selected two widely used Smac-XIAP complexes at this step because XIAP is the most characterized IAP and a direct inhibitor of initiator caspase-9 $(245,261)$. Apoptotic resistance was found to correlate with expression levels of XIAP in human prostate, melanoma, and non-small cell lung cancer cells (247). In addition, the ability of XIAP to inhibit apoptosis has been shown to allow melanoma cells to escape endoplasmic reticulum (ER) stress-mediated cell death (261). Hit compounds with best docking scores in either of the crystal structures were examined for their drug-like properties. Fifty hits from each of the two crystal structures (100 hits in total and their binding poses on the AVPI pocket are shown in Figure 4-2) were selected and combined to produce 71 unique final hits for subsequent in vitro assay against a panel of cancer cells.

\subsubsection{Validation of Virtual Screening Using In Vitro Anti-proliferation Assay}

Here we selected four well-established cancer cell lines (two human melanoma and two human prostate cancer) to first screen the hit compounds anti-proliferation potency. Those cell lines had been used widely to evaluate the efficacy of IAP inhibitors both in vitro and in vivo $(254,255,262)$. We first performed a two-concentration quick screening assay for the 71 unique hits identified in the previous step. Hits that displayed more than a $10 \%$ growth inhibition at a concentration of $3 \mu \mathrm{M}$ or $10 \mu \mathrm{M}$ on either of A375 or M14 melanoma cells after $48 \mathrm{~h}$ incubation are listed in the column diagram (Figure 4-3). Compounds UC-274, UC-476, UC-112, and UC-222 (Table 4-1, upper panel) achieved more than $50 \%$ growth inhibition in A375 or M14 cells at low micromolar concentration.

We subsequently determined the $\mathrm{IC}_{50}$ values for these four $\mathrm{UC}$ compounds and embelin (a reference IAP inhibitor $(246,254)$ serving as a positive control) on an expanded panel of human melanoma (A375 and M14) and prostate cancer (PC-3 and DU145) cell lines (Table 4-1, lower panel). Embelin is a potent and non-peptidic small molecular XIAP inhibitor with good in vivo antitumor and anti-inflammation activity. Among the four, UC-112 was the most active compound: its $\mathrm{IC}_{50}$ was as low as $0.7 \mu \mathrm{M}$ against PC-3 cell line. All four UC compounds had comparable or better antiproliferation activity than did embelin (Table 4-1). 


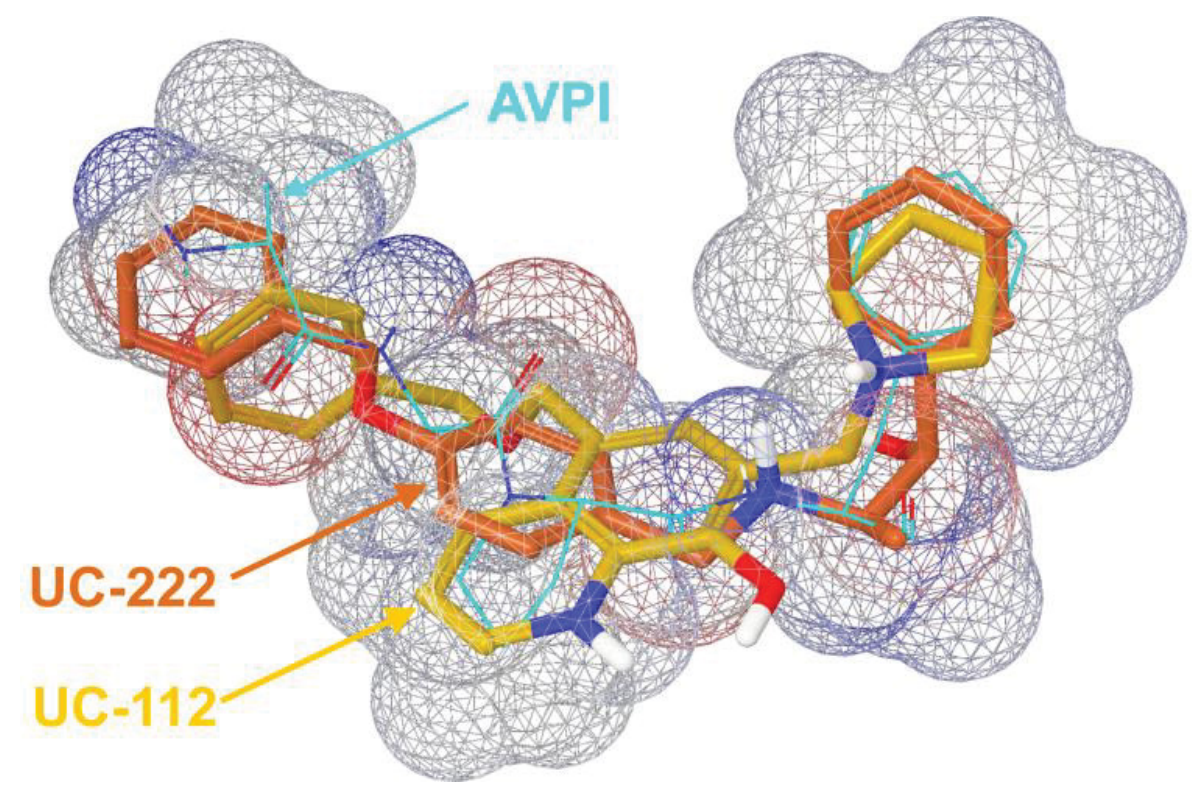

Figure 4-1. In silico similarity alignments of template ligand, Smac $\mathrm{N}$-terminus tetrapeptide AVPI (blue stick), with representative UC compounds (UC-222 in brown tube; UC-112 in yellow tube) 


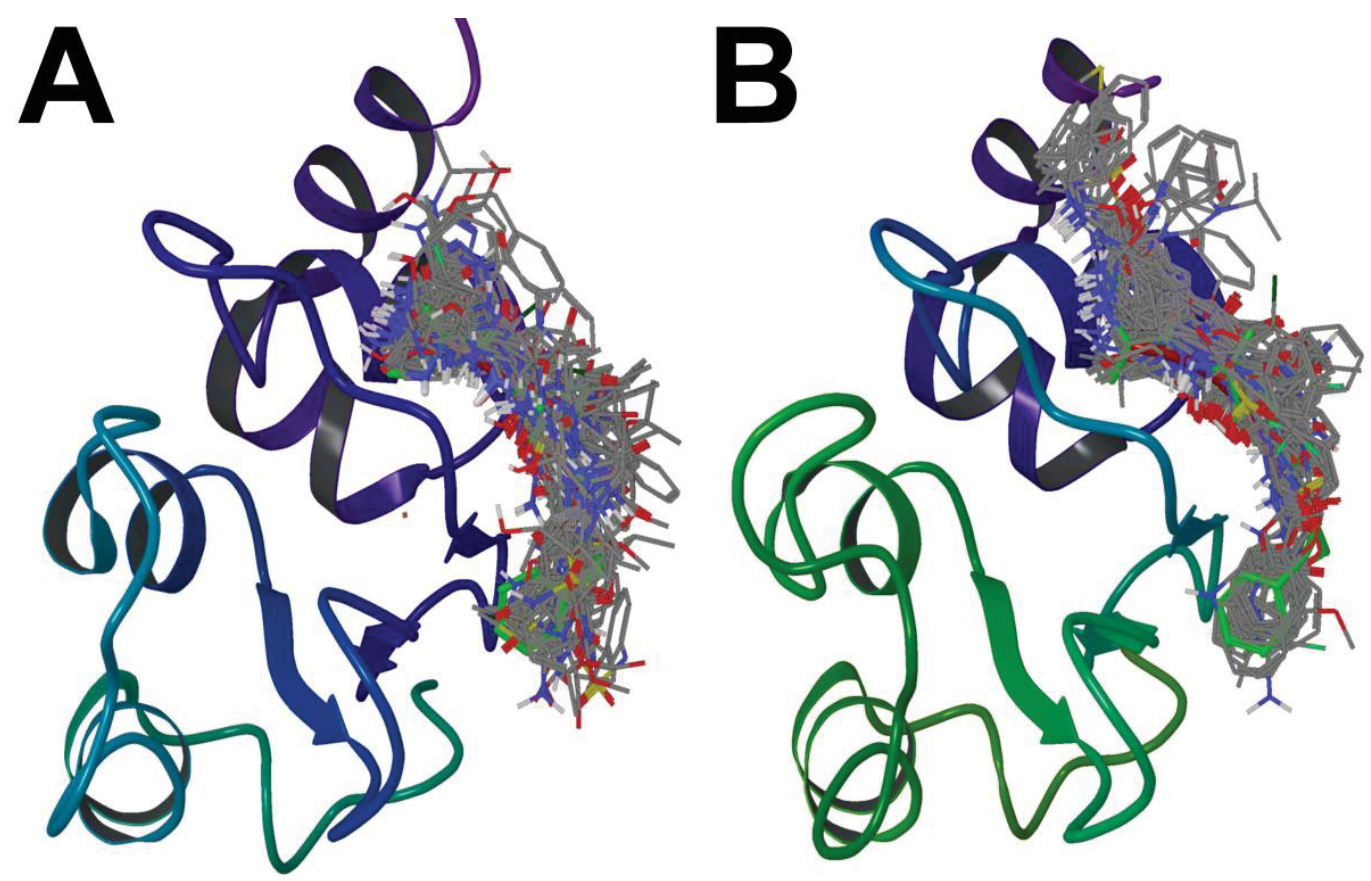

Figure 4-2. In silico predicted binding poses of 50 hits selected for in vitro activity evaluation docked into AVPI binding sides in different XIAP BIR3-Smac complexes PDB IDs: A, 1G73; B, 1TW6; hits were displayed with grey thin sticks and AVPI was presented with green tube stick. 


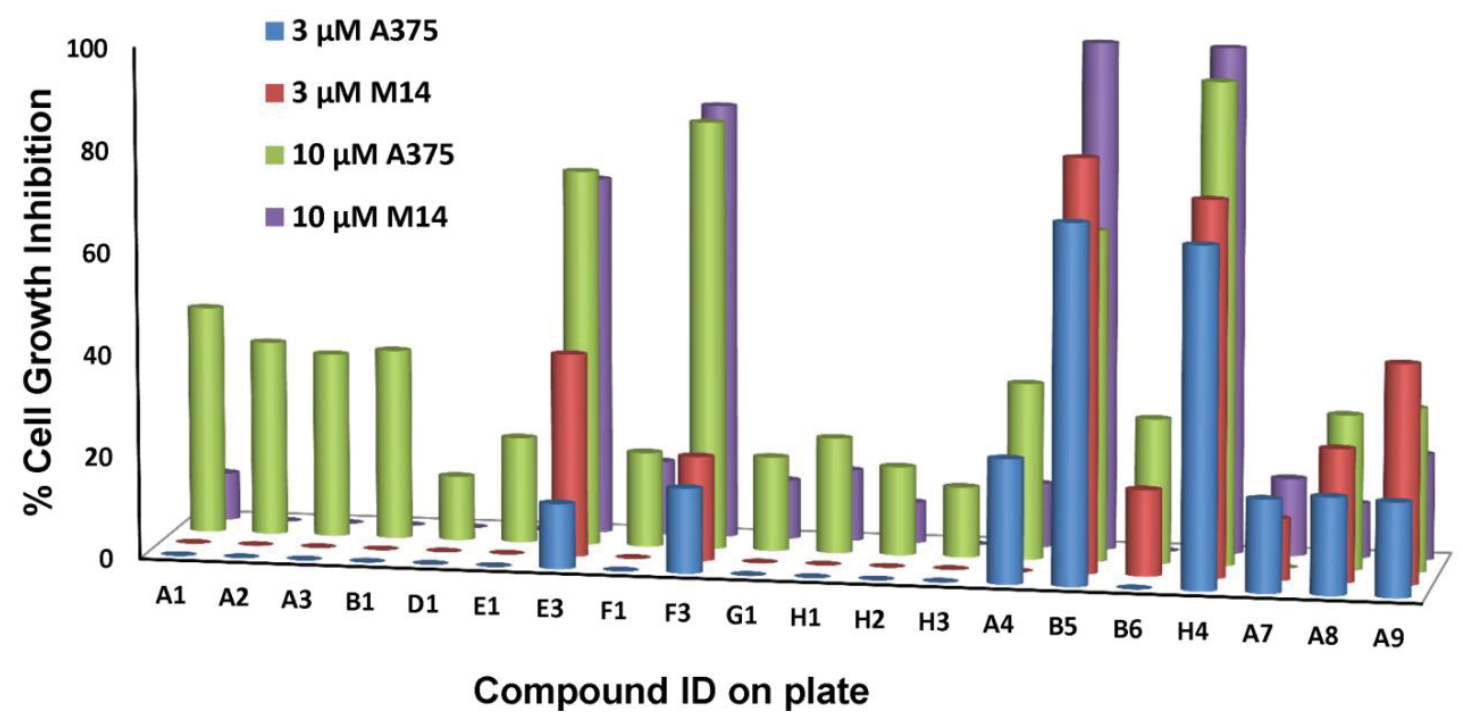

Figure 4-3. Cell growth inhibition percentages of XIAP inhibitor hits on human melanoma A375 and M14 cells at $4 \mu \mathrm{M}$ or $10 \mu \mathrm{M}$ after $48 \mathrm{~h}$ incubation determined by MTS assay $(n=4)$ 
Table 4-1. Growth inhibitory percentages of UC compounds at a concentration of $3 \mu \mathrm{M}$ or $10 \mu \mathrm{M}(\mathrm{MTS}$ assay, $\mathrm{n}=4)$ and $\mathrm{IC}_{50}(\mu \mathrm{M})$ values of $\mathrm{UC}$ compounds in comparison with embelin against cancer cell proliferation (SRB assay, $n=4$ )

\begin{tabular}{|c|c|c|c|c|c|c|c|c|c|c|}
\hline \multirow[b]{2}{*}{$\begin{array}{l}\text { Com- } \\
\text { pound } \\
\text { ID }\end{array}$} & \multirow[b]{2}{*}{$\begin{array}{l}\text { Struc- } \\
\text { ture }\end{array}$} & \multicolumn{2}{|c|}{$\begin{array}{c}\text { Growth } \\
\text { inhibition } \\
\text { of } \mathrm{A375} \text { cell } \\
(\%)\end{array}$} & \multicolumn{2}{|c|}{$\begin{array}{c}\text { Growth } \\
\text { inhibition } \\
\text { of } \mathrm{M} 14 \text { cell } \\
(\%)\end{array}$} & \multicolumn{5}{|c|}{$\mathrm{IC}_{50}(\boldsymbol{\mu M})$} \\
\hline & & $\begin{array}{c}3 \\
\mu \mathrm{M}\end{array}$ & $\begin{array}{c}10 \\
\mu \mathrm{M}\end{array}$ & $\begin{array}{c}3 \\
\mu \mathrm{M}\end{array}$ & $\begin{array}{c}10 \\
\mu \mathrm{M}\end{array}$ & $\begin{array}{c}\mathbf{A 3 7} \\
\mathbf{5}\end{array}$ & M14 & $\begin{array}{c}\text { PC- } \\
\mathbf{3}\end{array}$ & DU145 & $\underset{*}{\mathbf{A V G}}$ \\
\hline $\begin{array}{l}\text { UC- } \\
274\end{array}$ & & $\begin{array}{r}12.8 \\
\pm 3.5\end{array}$ & $\begin{array}{r}73.7 \\
\pm 2.4\end{array}$ & $\begin{array}{r}39.9 \\
\pm 3.3\end{array}$ & $\begin{array}{r}70.2 \\
\pm 1.1\end{array}$ & $\begin{array}{c}13.4 \\
\pm 0.7\end{array}$ & $\begin{array}{r}12.3 \\
\pm 0.4\end{array}$ & $\begin{array}{r}15.1 \\
\pm 0.3\end{array}$ & $\begin{array}{c}17.3 \pm \\
2.9\end{array}$ & $\begin{array}{r}14.5 \\
\pm 1.1\end{array}$ \\
\hline $\begin{array}{l}\text { UC- } \\
476\end{array}$ & & $\begin{array}{r}16.7 \\
\pm 2.3\end{array}$ & $\begin{array}{r}84.0 \\
\pm 1.6\end{array}$ & $\begin{array}{r}20.6 \\
\pm 2.0\end{array}$ & $\begin{array}{r}85.3 \\
\pm 3.8\end{array}$ & $\begin{array}{r}10.4 \\
\pm 0.2\end{array}$ & $\begin{array}{r}10.2 \\
\pm 0.2\end{array}$ & $\begin{array}{r}12.8 \\
\pm 2.1\end{array}$ & $\begin{array}{c}11.4 \pm \\
2.2\end{array}$ & $\begin{array}{r}11.2 \\
\pm 0.6\end{array}$ \\
\hline $\begin{array}{l}\text { UC- } \\
112\end{array}$ & & $\begin{array}{r}50.7 \\
\pm 5.1\end{array}$ & $\begin{array}{r}65.0 \\
\pm 3.4\end{array}$ & $\begin{array}{c}81.1 \\
\pm 1.7\end{array}$ & $\begin{array}{r}99.6 \\
\pm 0.6\end{array}$ & $\begin{array}{c}1.6 \pm \\
0.1\end{array}$ & $\begin{array}{c}2.5 \pm \\
0.3\end{array}$ & $\begin{array}{r}0.7 \pm \\
0.1\end{array}$ & $\begin{array}{c}3.4 \pm \\
0.8\end{array}$ & $\begin{array}{r}2.1 \pm \\
0.6\end{array}$ \\
\hline $\begin{array}{l}\text { UC- } \\
222\end{array}$ & & $\begin{array}{r}67.0 \\
\pm 1.6\end{array}$ & $\begin{array}{r}94.6 \\
\pm 1.0\end{array}$ & $\begin{array}{r}73.7 \\
\pm 2.0\end{array}$ & $\begin{array}{r}99.2 \\
\pm 0.7\end{array}$ & $\begin{array}{c}2.7 \pm \\
0.4\end{array}$ & $\begin{array}{c}3.5 \pm \\
0.1\end{array}$ & $\begin{array}{r}2.3 \pm \\
0.2\end{array}$ & $\begin{array}{c}4.5 \pm \\
1.7\end{array}$ & $\begin{array}{r}3.3 \pm \\
0.5\end{array}$ \\
\hline $\begin{array}{l}\text { Embe- } \\
\text { lin }\end{array}$ & & ND & ND & ND & ND & $\begin{array}{r}14.3 \\
\pm 3.2\end{array}$ & $\begin{array}{r}20.7 \\
\pm 5.4\end{array}$ & $\begin{array}{r}13.0 \\
\pm 1.8\end{array}$ & $\begin{array}{c}23.2 \pm \\
3.7\end{array}$ & $\begin{array}{r}17.8 \\
\pm 2.5\end{array}$ \\
\hline
\end{tabular}

ND: not determined. Data are shown as mean $\pm \mathrm{SEM}$. AVG*: average $\mathrm{IC}_{50}$ value was calculated using the data across all four tested cell lines of the same compound. 
We also investigated the cell toxicity of these UC compounds on two noncancerous cell lines (Table 4-2). Compared with its average $\mathrm{IC}_{50}$ value in the tested cancer cell lines, UC-112 has around 2.5 folds higher $\mathrm{IC}_{50}$ values on Hacat or HDFa cells. The $\mathrm{IC}_{50}$ values of UC-222 have increased 8 to 12 folds in the tested non-cancerous cells, compared with the average $\mathrm{IC}_{50}$ on the cancer cell lines.

\subsubsection{Hit Compounds Increased Caspase Activities Significantly Better than Embelin}

The Smac N-terminus IAP binding motif interacts with the IAPs and protects caspase function either directly or indirectly. Because the overexpression of IAPs in cancer cells result in the suppression of caspase functions, the capability of raising caspase activities is often used to measure the potency of IAP inhibitors (250-252). Embelin was reported to increase caspase activity in PC-3 cells at a concentration of 40 $\mu \mathrm{M}$ after 42-h incubation (254). However, our pilot study showed that four most potent UC compounds at the concentration of $40 \mu \mathrm{M}$ would kill most of the cancer cells, therefore making the measurement of caspase level below the assay detection limit. For this reason, we used much lower concentrations for UC compounds and performed the caspase functional Glo-assay (Figure 4-4A to D). All UC compounds effectively increased the caspase-3/7 (the executor caspases) and caspase-9 (the initiator caspase) levels on A375 and PC-3 cells in a time-dependent manner (Figure 4-4). In addition, UC222 at a concentration of $10 \mu \mathrm{M}$ and UC-112 at a concentration of $4 \mu \mathrm{M}(10 \mu \mathrm{M}$ of $\mathrm{UC}-$ 112 was too high for this assay due to its higher potency) had significantly stronger $(P<$ $0.01)$ caspase activation potency compared to embelin at 4 times or 10 times higher concentration $(40 \mu \mathrm{M})$.

\subsubsection{Western Blotting Analyses of IAPs Level Change in Cancer Cells}

Having established that the four UC hit compounds can potently increase the caspase activities, we asked the question whether these compounds produced their effects by directly inhibiting IAPs, since they were screened in silico as Smac N-terminus AVPI mimetic molecules. To answer this question, we studied the changes of IAP levels in cancer cells when they were treated by the two most potent UC compounds, UC-112 and UC-222, or the reference IAP inhibitor embelin. While there were no significant changes of XIAP, cIAP1, cIAP2, or survivin expression at short time incubation ( $3 \mathrm{~h}$, data not shown), when the incubation time was increased to $24 \mathrm{~h}, \mathrm{UC}-112$ at concentrations of 4 $\mu \mathrm{M}$ or $10 \mu \mathrm{M}$ (Figure 4-5A) significantly suppressed survivin level on A375, PC-3, and DU145 cell lines in a dose-dependent manner. UC-112 potently decreased survivin level (94\% and 97\% at $4 \mu \mathrm{M}$ and $10 \mu \mathrm{M}$, respectively) in A375 cells while its inhibition of the other major IAPs (i.e., XIAP, cIAP1, cIAP2), which also interact with Smac, was much weaker. At the same time point $(24 \mathrm{~h}), \mathrm{UC}-222$ at concentrations of $4 \mu \mathrm{M}$ or $10 \mu \mathrm{M}$ decreased the level of cIAP2 on PC-3 cells and XIAP on DU145 cells but only had very mild effects on the survivin level in either cell line (Figure 4-5A). 
Table 4-2. $\quad \mathrm{IC}_{50}(\boldsymbol{\mu M})$ values of $\mathrm{UC}$ compounds in comparison with embelin against normal cells proliferation (SRB assay, $n=4)$

\begin{tabular}{lcccccc}
\hline $\begin{array}{l}\text { Cell line } \\
\text { type }\end{array}$ & $\begin{array}{c}\text { Cell line } \\
\text { name }\end{array}$ & UC-274 & UC-476 & UC-112 & UC-222 & Embelin \\
\hline $\begin{array}{l}\text { Cancer } \\
\text { cell lines }\end{array}$ & AVG* & $14.5 \pm 1.1$ & $11.2 \pm 0.6$ & $2.1 \pm 0.6$ & $3.3 \pm 0.5$ & $17.8 \pm 2.5$ \\
$\begin{array}{l}\text { Non- } \\
\text { cancerous } \\
\text { cell lines }\end{array}$ & Hacat & $>100$ & $6.2 \pm 1.8$ & $5.6 \pm 1.5$ & $36.0 \pm 2.3$ & $43.6 \pm 3.7$ \\
\hline
\end{tabular}

Data are shown as mean $\pm \mathrm{SEM}$. $\mathrm{AVG}^{*}$ : average $\mathrm{IC}_{50}$ value on four cancer cell lines (A375, M14, PC-3, DU145). 

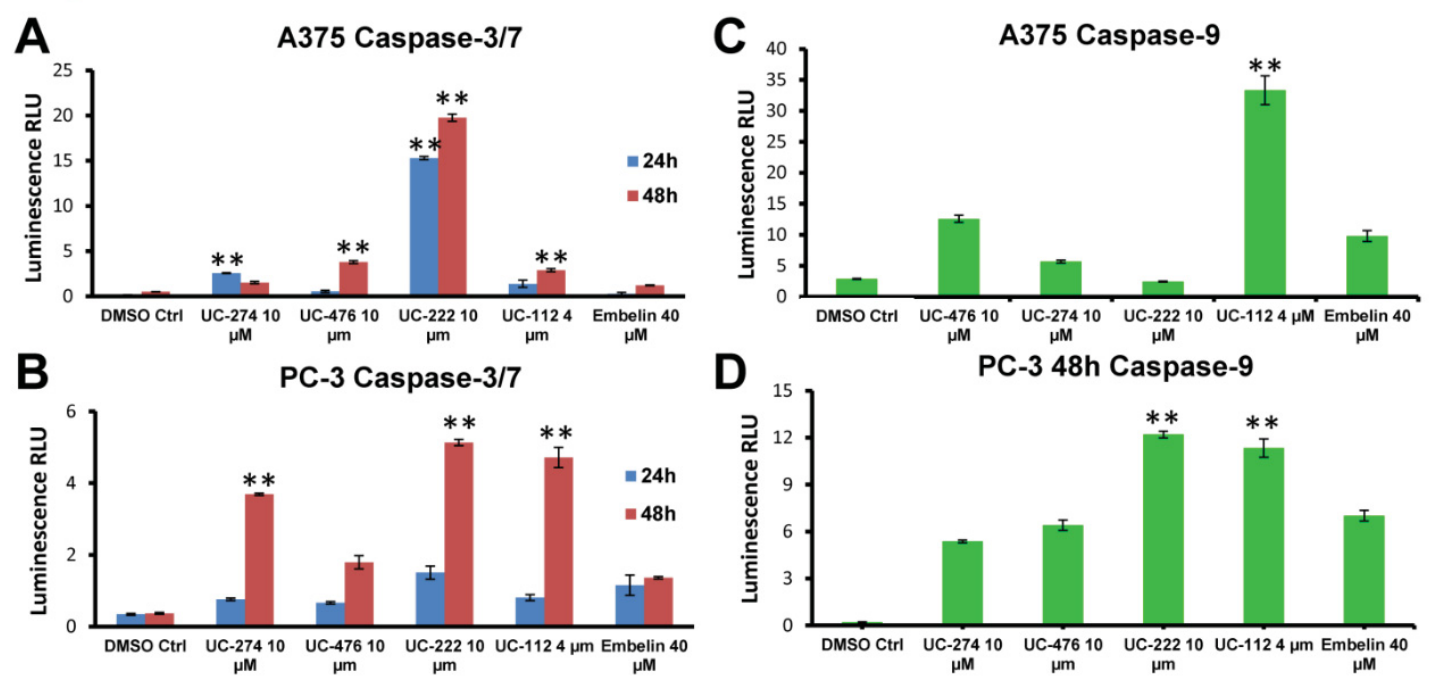

Figure 4-4. Relative in vitro caspase activity of human melanoma A375 or human prostate cancer PC-3 cells after treatment with UC compounds or embelin at different concentrations $(n=4)$

Testing concentrations ( $4 \mu \mathrm{M}$ or $10 \mu \mathrm{M})$ of UC compounds were selected in reference to their in vitro anti-proliferation potency. All the relative light unit (RLU) data were normalized according to the cell viability results read from the same well in a 96-well plate. A, caspase-3/7 in A375 cells. B, caspase-3/7 in PC-3 cells. C, caspase-9 in A375 cells (48 hr incubation). D, caspase-9 in PC-3 cells (48 hr incubation). $* * P<0.01$ compared to corresponding results of embelin group. 


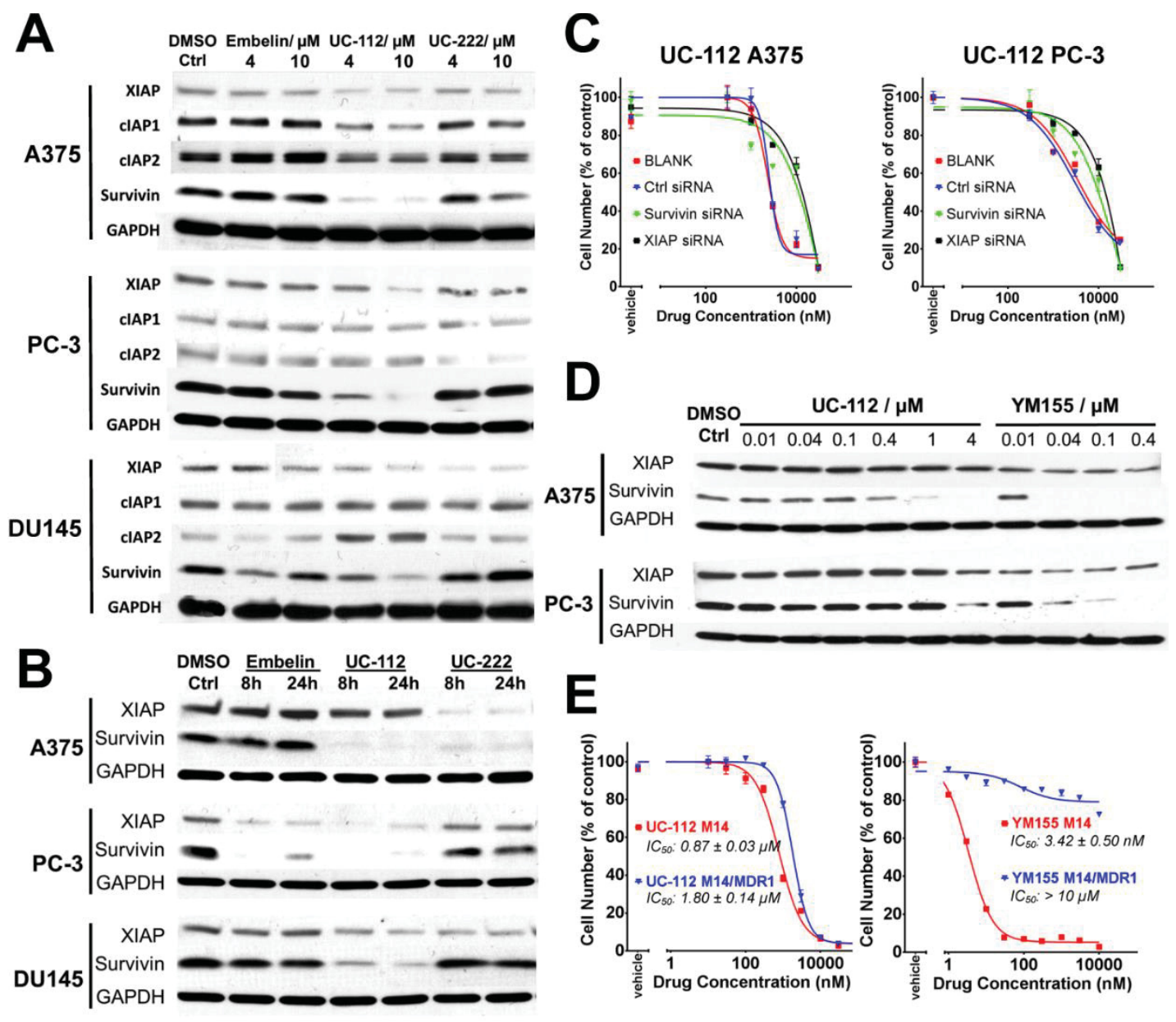

Figure 4-5. Compounds UC-112 and UC-222 down-regulate the IAP levels in vitro A, Western blotting analysis using cell lysates of A375, PC-3, or DU145 cells that have been treated by compounds at concentrations of 4 or $10 \mu \mathrm{M}$ for $24 \mathrm{~h}$. B, at concentration of $40 \mu \mathrm{M}$, Western blotting analysis showed that UC-112 and UC-222 can effectively induce degradation of XIAP and survivin, after 8-h or 24-h incubation. C, Silencing survivin or XIAP gene in A375 or PC-3 cells increased the $\mathrm{IC}_{50}$ values of UC-112. Treatment started at $24 \mathrm{hr}$ after the siRNA transfection. The cell viability after $48 \mathrm{hr}$ treatment was determined using MTS assay $(n=4)$. D, comparison between compound UC-112 and YM155 regarding the potency of reduced survivin level in A375 or PC-3 cells. Photographs show representative data from three independent experiments. E, MTS assay $(n=4)$ revealed that UC-112 overcomes Pgp-mediated multidrug resistance much better than does YM155. 
In comparison, the inhibition effects of embelin at low concentrations ( $4 \mu \mathrm{M}$ and $10 \mu \mathrm{M}$ ) on the IAP levels were not clear in these cell lines (Figure 4-5A). When we increased its concentration to $40 \mu \mathrm{M}$, embelin could inhibit XIAP expression in PC-3 cells with an 8-h incubation (Figure 4-5B), consistent with literature report (254). In contrast, UC-112 at $40 \mu \mathrm{M}$ only moderately decreased the level of XIAP but substantially down-regulated level of survivin in A375 cells (Figure 4-5B). UC-222 at this concentration $(40 \mu \mathrm{M})$ was most potent in decreasing XIAP levels in A375 and DU145 cells but PC-3 cells were more resistant to the UC-222 treatment. Thus, UC-112 demonstrated selective inhibition against survivin among the IAPs tested (Figure 4-6).

To validate whether the toxicity of UC-112 on cancer cells results from its downregulation effects on IAP levels, we silenced the gene of survivin or XIAP by transfecting A375 and PC-3 cells with specific siRNAs. Then the anti-proliferation efficacy of UC112 on these transfected cancer cells was determined with MTS assay and the data was shown in Figure 4-7. Compared to the results from the non-transfected (blank group) or non-specific siRNA transfected cells, $\mathrm{IC}_{50}$ values of UC-112 increased 7 to 10 folds when either XIAP or survivin expression was silenced.

Survivin is a unique and smallest member of inhibitor of apoptosis proteins (IAPs). It is highly expressed in most types of cancer but has very low or undetectable expression in differentiated normal tissues $(161,194,263-266)$. Consistent with this differential expression, survivin inhibitors have been shown to have broad efficacy in many types of cancer and low toxicity $(157,166)$. Additionally, survivin expression has been well correlated with tumor progression, resistance to existing therapies, and poor patient survival $(231,263,267,268)$. Survivin is involved in a myriad of oncogenic pathways and is considered to be a nodal protein. Recent studies have also revealed that high levels of survivin positively correlate with the over-activation of known oncogenic pathways (HIF-1 $\alpha(157,231)$, HSP90 ${ }^{(158,159),}$ PI3K/AKT $(160,161)$, ERK (162), Bcl-2 $(269,270)$ and RAS pathways $(163,164))$ but negatively correlate with tumor suppressor genes (p53, PTEN (165-167)). Together, these characteristics make selective inhibition of survivin an ideal target for novel cancer drug discovery.

Encouraged by the results that compound UC-112 is a relatively potent inhibitor of survivin, we quantified the potency of the compound by incubating A375 and PC-3 cells with serially diluted UC-112 solutions (from $4 \mu \mathrm{M}$ to $10 \mathrm{nM}$ ). We also selected YM155 (204, 206, 207, 262), a highly efficient survivin promoter inhibitor as the experiment's positive control. Results (Figure 4-5D, Figure 4-6B) showed that UC-112 significantly down-regulated survivin levels ( $>50 \%$ compared to control group) on A375 cells at a concentration of $400 \mathrm{nM}$ in $24 \mathrm{~h}$, while a higher concentration $(1 \mu \mathrm{M})$ or longer treatment time (48 h) (data not shown) was needed in PC-3 cells. YM155 showed to be a more potent survivin inhibitor and strongly inhibited the expression of survivin at concentration as low as $40 \mathrm{nM}$ in A375 cells or $100 \mathrm{nM}$ in PC-3 cells, consistent with data in published reports(204, 207). No significant change in XIAP levels was observed in either cell line treated with either UC-112 or YM155 at any concentrations tested. This observation confirmed that compound UC-112 could selectively decrease the survivin protein level over the levels of other IAP family proteins including the XIAP. Although 

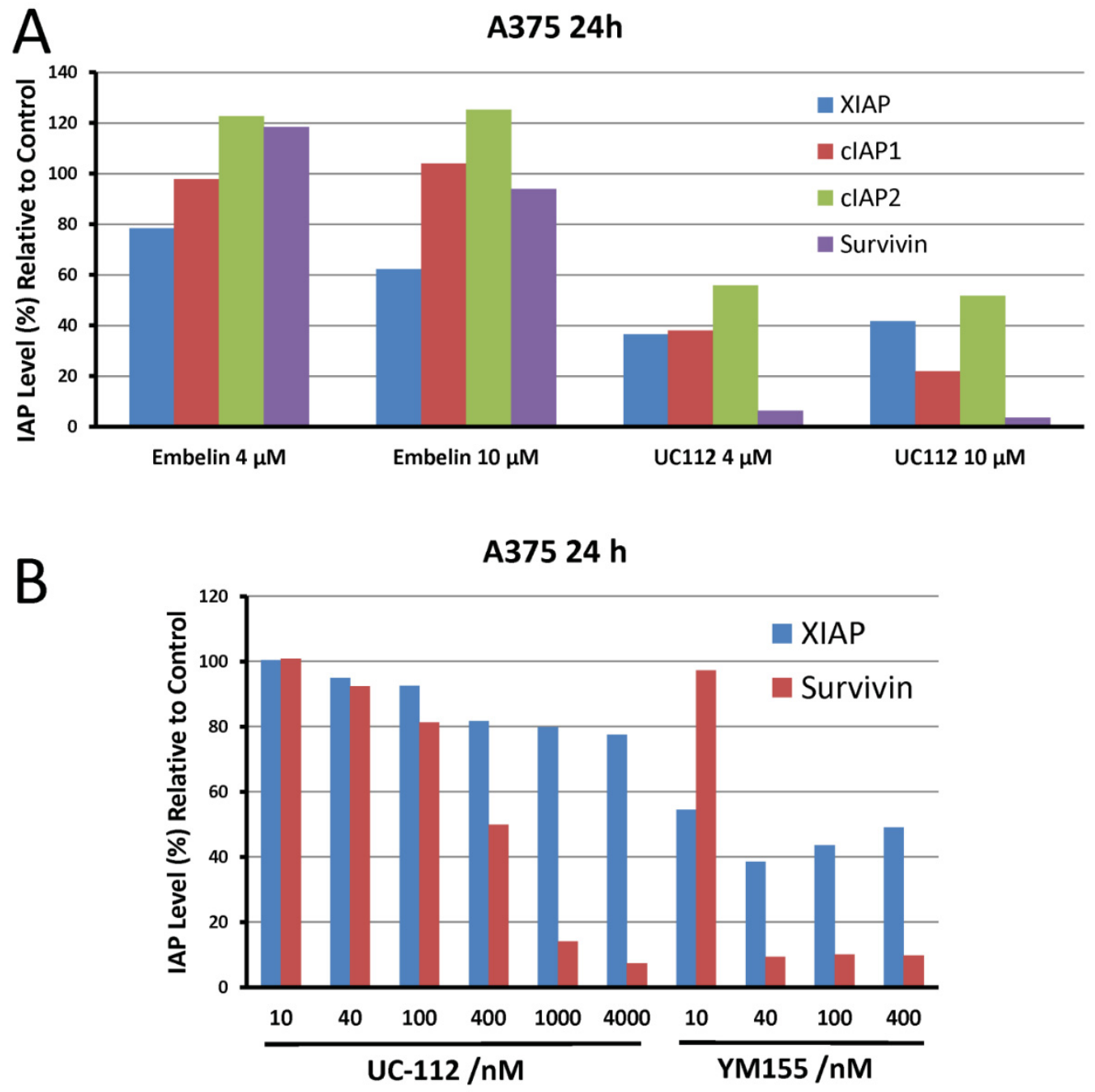

Figure 4-6. Lane density quantification data of western blotting analysis results (A375 cells) in (A) Figure 4-5A and (B) Figure 4-5C 


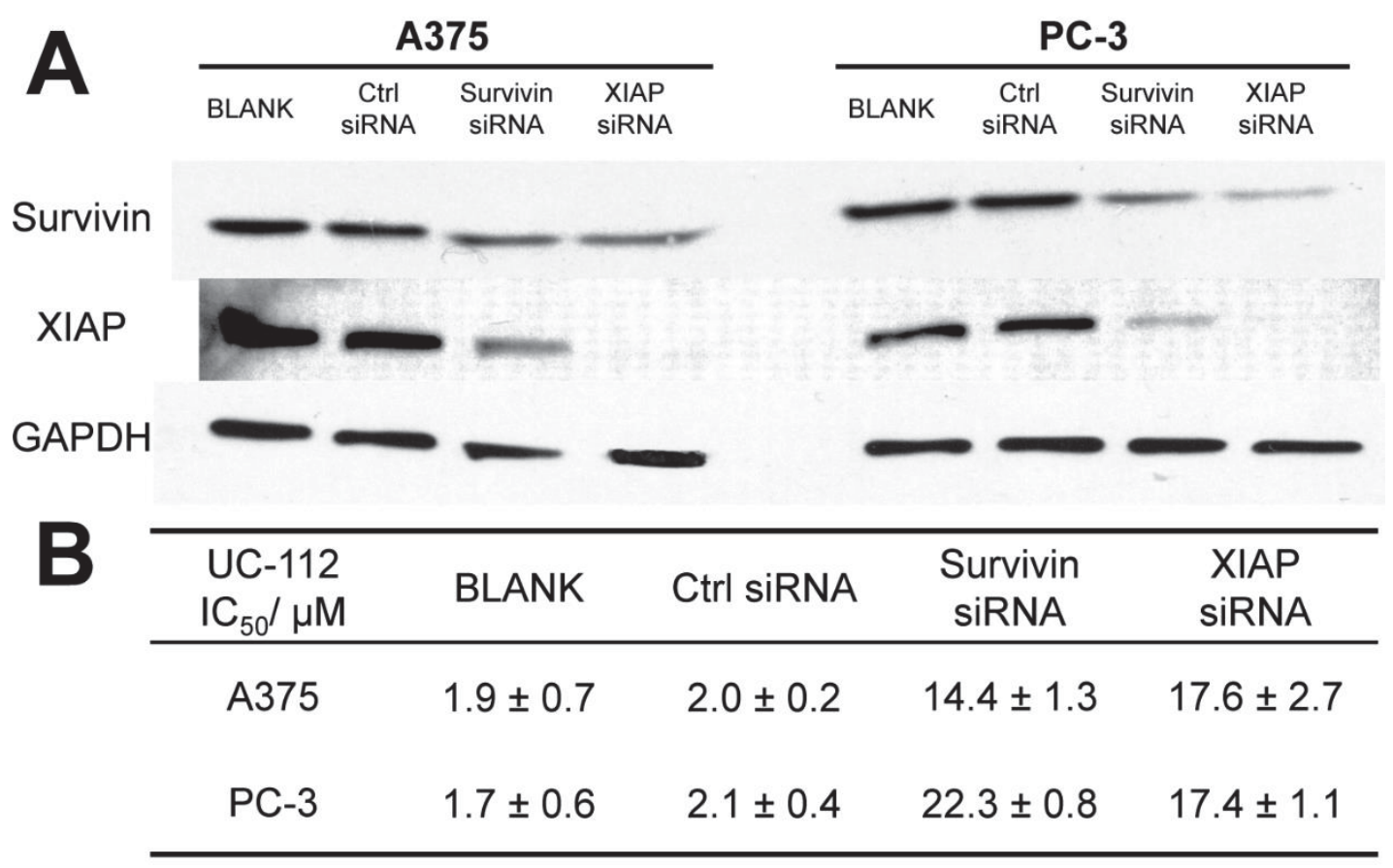

Figure 4-7. IC IC $_{50}$ values of UC-112 increased in survivin or XIAP gene silenced A375 and PC-3 cells

A, western blotting showed that siRNA transfection specifically silenced the expression of survivin or XIAP in A375 and PC-3 cells. B, IC I0 $_{50}$ values of UC-112 tested on different siRNA transfected cells in MTS assay $(n=4)$. 
YM155 is extremely potent in binding and inhibiting the survivin promoter, its main drawback is that it is a substrate for the Pgp drug efflux pump (271). In fact, when tested in Pgp overexpressed M14/MDR1 melanoma cells, UC-112 have comparable $\mathrm{IC}_{50}$ values in the resistant M14/MDR1 and the sensitive M14 parental cells (Figure 4-5E and Table 4-3, small resistance index is desirable to overcome drug resistance), displaying a small resistance index value around 2. In contrast, the resistance index of YM155 was larger than 2900, consistent with the finding that it is a Pgp substrate.

\subsubsection{Proteasome Inhibitor MG132 Rescued the Down-regulated Survivin Levels in Cancer Cells Treated by UC-112}

Although the degradation mechanisms of survivin still need to be clearly delineated, XIAP-associated factor 1 (XAF1) was reported to activate E3 activity in XIAP and directly led to survivin degradation through ubiquitination (172). To further understand the possible mechanism leading to survivin down-regulation by UC-112, we hypothesized that this compound involved the ubiquitin-related degradation process of survivin. To test this hypothesis, we determined whether the addition of a panproteasome inhibitor, MG132, would counteract the ubiquitin-mediated degradation process and rescue survivin from the action of UC-112. As shown in Figure 4-8A, coincubation of pan-proteasome inhibitor MG132 $(10 \mu \mathrm{M})$ with UC-112 for $24 \mathrm{~h}$ effectively rescued the survivin levels in PC-3 and DU145 cells. MG132 at $10 \mu \mathrm{M}$ in this study was unable to rescue survivin in A375 cells because these cells are more sensitive to the survivin down-regulation effect resulted from UC-112 treatment. However, if A375 cells were pretreated with higher concentrations of MG132 $(20 \mu \mathrm{M}$ or $50 \mu \mathrm{M})$ for 6 $\mathrm{h}$ followed by incubation with UC-112 for another $24 \mathrm{~h}$ after washing away MG132, the survivin level could be rescued from UC-112 treatment (Figure 4-8B). The results suggest that UC-112 may produce its survivin inhibition effect, at least in part, via the ubiquitin-mediated degradation of survivin.

\subsubsection{UC-112 Arrested A375 Melanoma Cells in $G_{1}$ Phase}

It is well established that survivin plays complex and critical roles during cell mitosis as a chromosomal passenger protein to promote chromosome segregation and cytokinesis in the $\mathrm{G}_{2} / \mathrm{M}$ phase of the cell cycle $(194,264,267)$. Survivin as a cytoplasmic protein is dominantly expressed in the $\mathrm{G}_{2} / \mathrm{M}$ phase, followed by a rapid degradation via the ubiquitin-proteasome pathway during $\mathrm{G}_{1}(161,170,264,267)$. Therefore, the survival of cancer cells in the $\mathrm{G}_{2} / \mathrm{M}$ phase is highly dependent on the presence of survivin, while cells in the $\mathrm{G}_{1}$ phase are insensitive to survivin expressions. If $\mathrm{UC}-112$ potently degrades survivin in cancer cells, then we would expect that cells in the $\mathrm{G}_{2} / \mathrm{M}$ phase will collapse while the less survivin-dependent cells in the $\mathrm{G}_{1}$ phase will remain, leading to an overall $\mathrm{G}_{1}$ phase accumulation upon UC-112 treatment. To test this hypothesis, we performed cell cycle analysis to determine how UC-112 would influence the cell cycle distribution. 
Table 4-3. UC-112 overcomes Pgp-mediated drug resistance much better than YM155 (n = 4)

\begin{tabular}{lccc}
\hline & \multicolumn{2}{c}{ IC $_{\mathbf{5 0}}$} & \\
\cline { 2 - 3 } Compound ID & M14 (nM) & M14/MDR1 (nM) & Resistant Index \\
\hline UC-112 & $780 \pm 32$ & $1798 \pm 140$ & 2.3 \\
YM155 & $3.4 \pm 0.5$ & $>10,000$ & $>2,941$ \\
Colchicine & $5.0 \pm 0.5$ & $345 \pm 12$ & 69 \\
\hline
\end{tabular}

Resistant Index was calculated by dividing the $\mathrm{IC}_{50}$ in resistant cells by the $\mathrm{IC}_{50}$ in the sensitive parental cells. Higher RI values suggests stronger drug resistance 


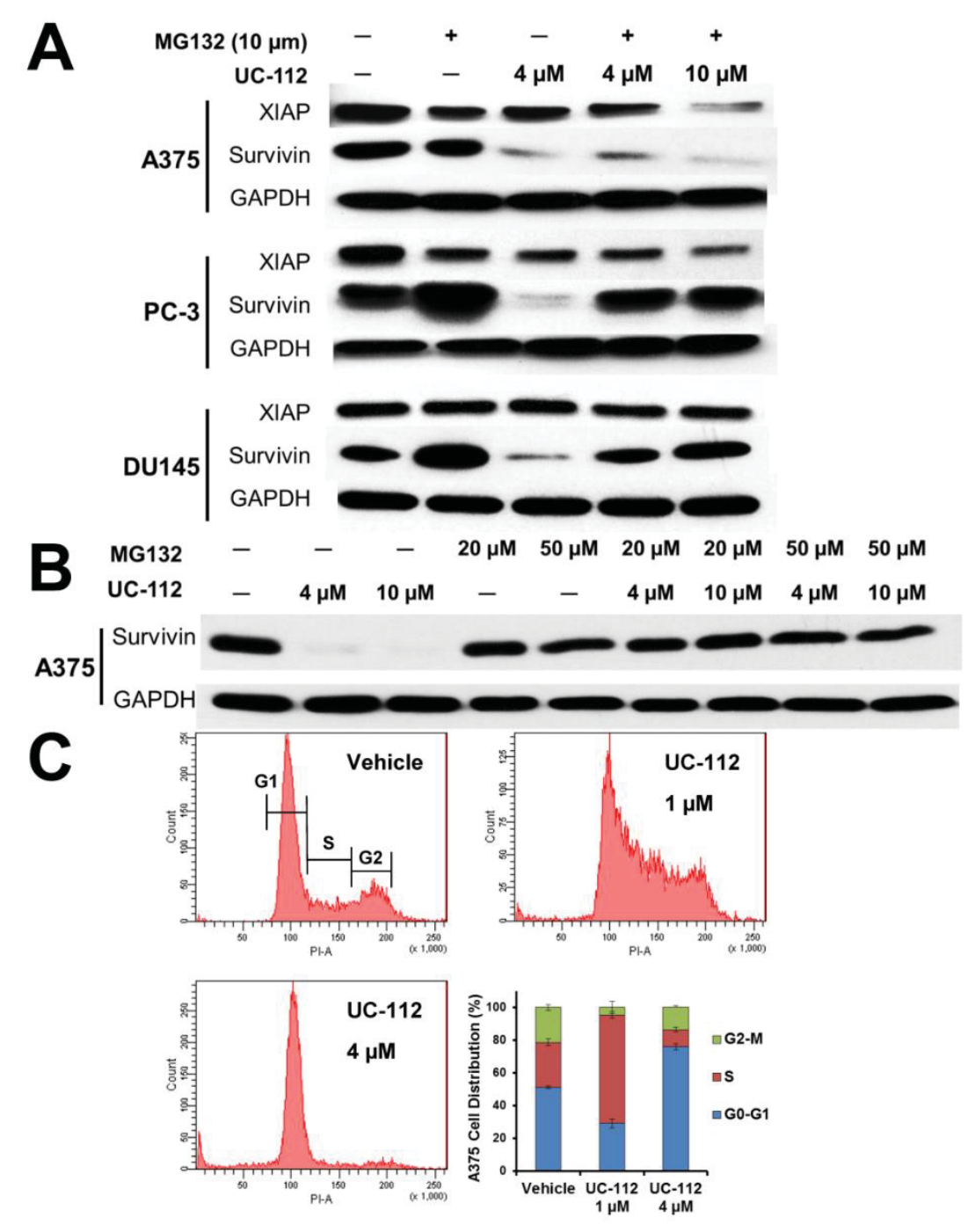

Figure 4-8. Proteasome inhibitor MG132 can rescue survivin inhibition by UC112

A, Western blotting analysis after co-incubation of MG132 $(10 \mu \mathrm{M})$ with UC-112 for 24 $\mathrm{h}$ in A375, PC-3, and DU145 cells. B, A375 cells were pretreated by high doses of MG132 $(20 \mu \mathrm{M}$ or $50 \mu \mathrm{M})$ for $6 \mathrm{~h}$ and washed once by blank cell culture medium. Then UC-112 was added for following $24 \mathrm{~h}$ incubation. Photographs showed representative data from three independent experiments. C, cell cycle analysis of A375 cells incubated with $1 \mu \mathrm{M}$ or $4 \mu \mathrm{M} \mathrm{UC}-112$ for $24 \mathrm{~h}(n=3)$. 
The results (Figure 4-8C) of flow cytometry analysis showed that incubation with $1 \mu \mathrm{M}$ UC-112 for $24 \mathrm{~h}$ caused A375 cells to accumulate in the S phase, with a concurrent reduction of cells in the $\mathrm{G}_{2} / \mathrm{M}$ phase. When the concentration of UC-112 increased to 4 $\mu \mathrm{M}$, a large percentage of $\mathrm{A} 375$ cells $(76 \pm 2 \%$ in treatment vs. $51 \pm 1 \%$ in vehicle control) accumulated in the $G_{0} / G_{1}$ phase with escalated sub- $G_{1}$ events, which indicated induction of cell apoptosis. The percentage of cells remaining in the $\mathrm{G}_{2} / \mathrm{M}$ phase was decreased (13 $\pm 1 \%$ in treatment vs. $21 \pm 2 \%$ in vehicle control). In this study, UC-112 blocked the A375 cell cycle in $\mathrm{G}_{1}$ phase in a dose-dependent manner. This observation is consistent with the proposed mechanisms of action for UC-112 as described above, providing additional evidence that UC-112 targets survivin degradation, a posttranslational modification process. In contrast, down-regulation of survivin gene expression by YM155 was a cell cycle-independent event without $\mathrm{G}_{1}$ cell arrest in a wide panel of cancer cells (272). Thus, unlike YM155, UC-112 down-regulated the survivin levels in cancer cells more likely by promoting the ubiquitin-mediated degradation of survivin rather than by altering the expression of survivin at the transcription level.

\subsubsection{UC-112 Inhibited Tumor Growth in a Melanoma Xenograft Model In Vivo}

To further investigate the therapeutic potential of compound UC-112, we evaluated its anti-tumor efficacy in an A375 xenograft mouse model. We first estimated the maximum tolerable dose (MTD) in mice. UC-112 was well tolerated in mice up to $200 \mathrm{mg} / \mathrm{kg}$ which was the highest dose we tested. The very low toxicity displayed by UC112 is consistent with the above evidence suggesting that UC-112 is a selective survivin inhibitor, because survivin is highly expressed in tumor cells but minimally expressed in normal tissues $(157,263,266)$. Considering realistic doses that are normally used clinically, we decreased the dose of UC-112 to $10 \%(20 \mathrm{mg} / \mathrm{kg})$ and $20 \%(40 \mathrm{mg} / \mathrm{kg})$ in subsequent in vivo efficacy studies. After 3 weeks of continuous treatment, UC-112 was highly effective in inhibiting melanoma tumor growth in the A375 xenograft model in a dose-dependent pattern (Figure 4-9A and Figure 4-10) without causing $(>10 \%)$ body weight loss in nude mice (Figure 4-9B). Treatment with UC-112 at $20 \mathrm{mg} / \mathrm{kg}$ achieved $65.59 \pm 19.56 \%$ tumor growth inhibition (TGI) compared to vehicle control group, while the dose at $40 \mathrm{mg} / \mathrm{kg}$ almost completely inhibited tumor growth (TGI was $95.23 \pm$ $3.11 \%$ ). The average tumor weight in the UC-112 $40-\mathrm{mg} / \mathrm{kg}$ treatment group was only $13.56 \%$ of that in the vehicle control group (Figure 4-9C). To determine whether the potential mechanisms of action for UC-112 observed in vitro will remain in vivo, we compared the IAP protein expressions between the control and treatment tumors. As shown in Figure 4-9D, UC-112 significantly reduced both survivin and XIAP levels in the lysates of fresh tumors isolated at the end of the treatment, confirming the in vivo efficacy of UC-112 was at least in part through the strong inhibition of IAPs.

Hematoxylin and eosin staining showed that UC-112 induced extensive tumor cell death in these A375 melanoma tumors (Figure 4-9E). Furthermore, TUNEL assay which measured nuclear DNA fragmentation (Figure 4-9F) clearly showed the dose-dependent increasing of apoptosis in tumor sections of UC-112 treatment groups. All these results are consistent with our in vitro observations that UC-112 could efficiently inhibit tumor cell proliferation by down-regulating the level of IAPs, especially survivin protein. 


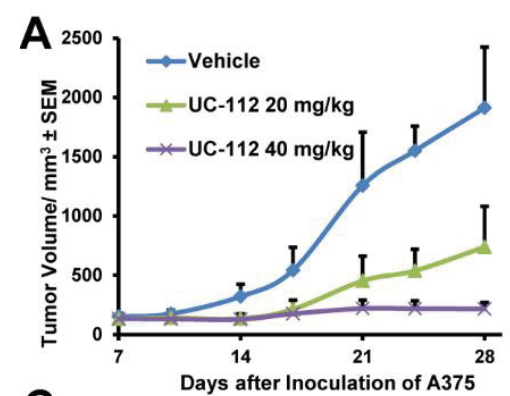

C
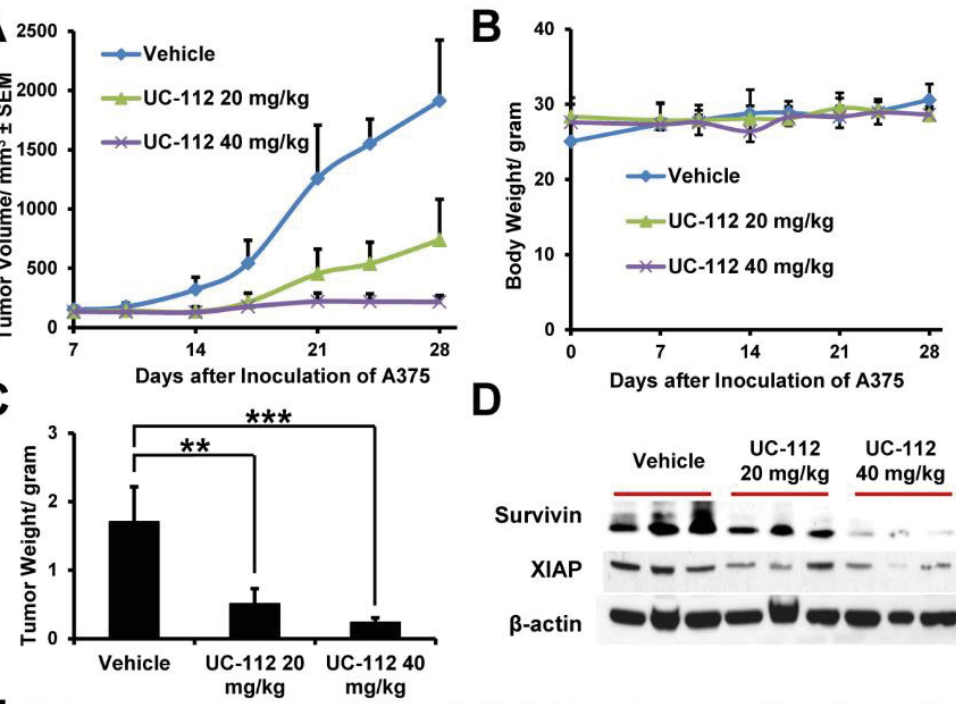

D
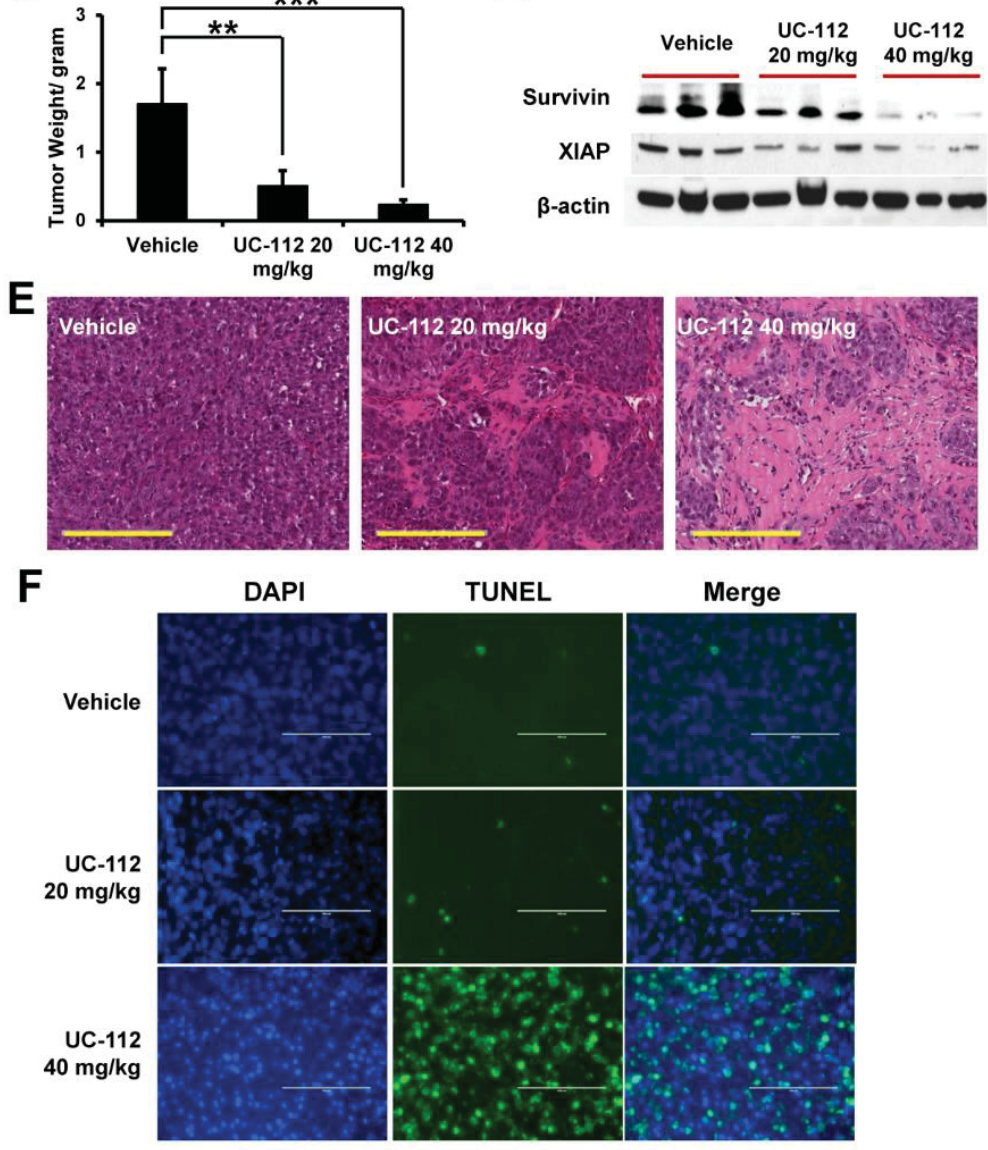

TUNEL

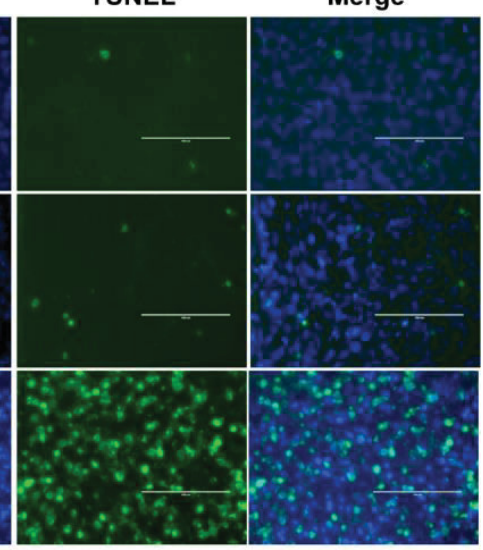

Figure 4-9. UC-112 (20 mg/kg and $40 \mathrm{mg} / \mathrm{kg})$ strongly suppress melanoma tumor growth in an A375 xenograft mouse model ( $n=7$, i.p. injection 5 days per week) A, tumor volume growth curve. B, mice body weight versus time plot. C, tumor weight comparison bar graph. $* * P<0.01, * * * P<0.001$ compared to the corresponded results of vehicle group. D, Western blotting analysis of survivin and XIAP levels in fresh tumor lysates. Each lane represents tumor tissue lysate from a single mouse. E, representative immunohistochemistry images for $\mathrm{H} \& \mathrm{E}$ staining of A375 tumor tissue sections. The scale bar (yellow) in each image represents $200 \mu \mathrm{m}$. Photographs show representative data from three independent experiments. F, representative fluorescence microscope images for TUNEL assay of A375 tumor tissue sections. The scale bar (white) in each image represents $100 \mu \mathrm{m}$. 


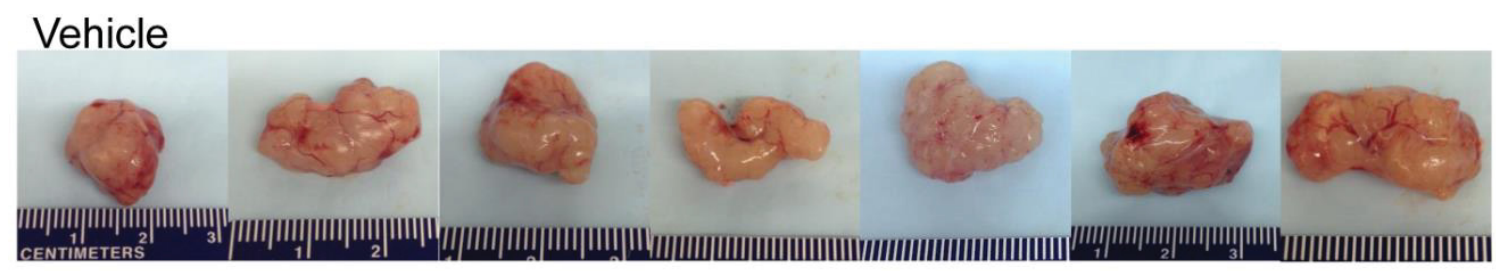

UC-112 $20 \mathrm{mg} / \mathrm{kg}$

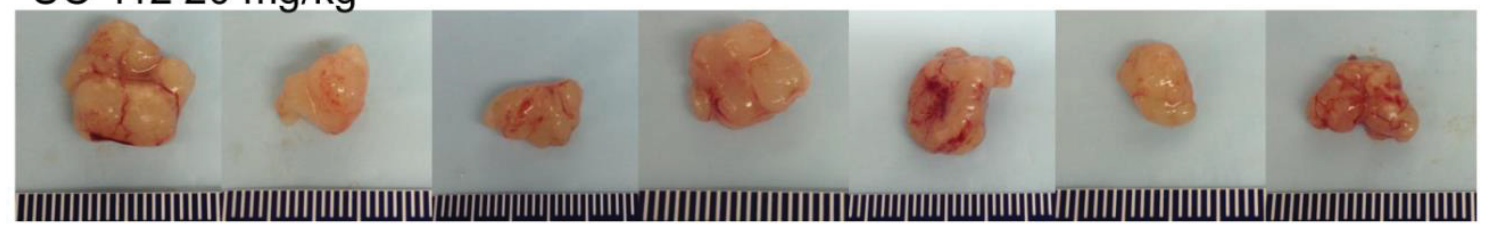

UC-112 $40 \mathrm{mg} / \mathrm{kg}$

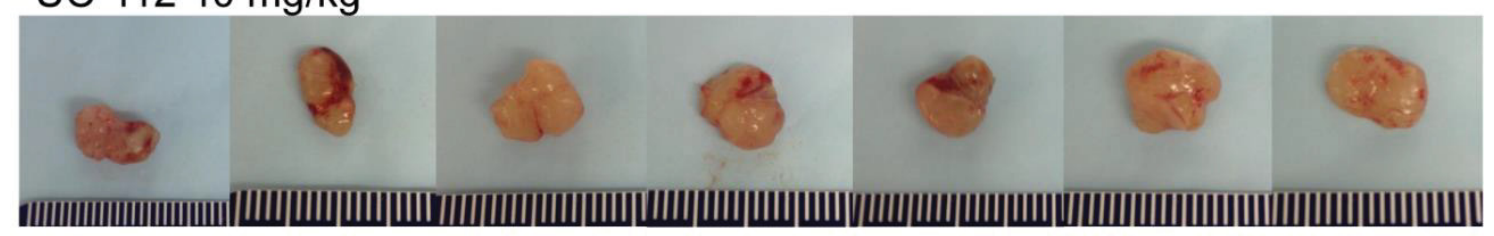

Figure 4-10. Tumor images of in vivo anti-tumor efficacy evaluation of UC-112 (20 $\mathrm{mg} / \mathrm{kg}$ and $40 \mathrm{mg} / \mathrm{kg}$ ) on $\mathrm{A375}$ xenograft nude mice model ( $n=7$, i.p. injection five days per week for three continuous weeks) 


\section{CHAPTER 5. ANTI-CANCER EFFICACY, PHYSIO-CHEMICAL PROPERTY AND TARGET VALIDATION STUDIES OF OPTIMIZED UC-112 ANALOGS*}

\subsection{Introduction}

We recently discovered that UC-112, [5-((benzyloxy)methyl)-7-(pyrrolidin-1ylmethyl)quinolin-8-ol], is a potent, selective survivin inhibitor (273). UC-112 inhibits tumor cell growth in several cancer cell lines in vitro and suppresses melanoma tumor growth in vivo (273). Mechanistic studies indicated that UC-112 selectively inhibits survivin expression and induces strong cancer cell apoptosis. By performing targeted structural modifications, a series of novel UC-112 analogs were obtained. Biological evaluation of these new analogs revealed their excellent anti-proliferative activity against several cancer cell lines including multidrug-resistant phenotypes. Mechanism of action studies confirmed that these new UC-112 analogs maintained their mechanisms of actions by selectively down-regulating the level of survivin among other IAP family proteins. Preliminary in vivo evaluation for the most active compound MX106 demonstrated its efficacy against human melanoma tumor growth.

\subsection{Materials and Methods}

\subsubsection{Cell Culture and Cell Viability Assay}

The anti-proliferative effect of UC-112 and its analogs were tested in human melanoma (A375, M14 and M14/LCC6MDR1) and human prostate cancer (PC-3) cell lines. All the cell lines were purchased from ATCC (American Type Culture Collection, Manassas, VA). The cancer cells were cultured using the supplemented cell culture medium as described before $(259)$ at $37^{\circ} \mathrm{C}$ in a humidified atmosphere containing $5 \%$ $\mathrm{CO}_{2} .5000$ cells in logarithm growing phase were seeded overnight into each well of a 96-well plate. Then the cells were continuously incubated with sequential diluted compound solution ( $10 \mathrm{nM}$ to $100 \mu \mathrm{M}, 100 \mu \mathrm{l}$ per well) in cell culture medium. After 48 $\mathrm{h}$ treatment, the cell viability was determined in MTS assay and $\mathrm{IC}_{50}$ was calculated $(n=$ $4)$ as described before $(127,273)$.

\subsubsection{NCI-60 Screening}

Four compounds including the parental compound UC-112 were submitted to National Cancer Institute for its NCI-60 cell line screening, initially tested at one

* Modified with permission. Xiao M, Wang J, Lin Z, Lu Y, Li Z, White S, Miller D, Li W. Design, synthesis and structure-activity relationship studies of novel survivin inhibitors with potent anti-proliferative properties. PLOS ONE. DOI:

10.1371/journal.pone.0129807 
concentration $(10 \mu \mathrm{M})$, and subsequently selected for full five concentration testing following the standard protocols disclosed by NCI (274). In brief, cells were plated into 96 well micro-titer plates $24 \mathrm{~h}$ prior to the treatment of compound solution for $48 \mathrm{~h}$, then the cell viability was read out through absorbance of sulforhodamine B (SRB) staining.

\subsubsection{Western Blotting}

Lysates of A375 cells treated by the compound solution for $24 \mathrm{~h}$ were used to determine the change of IAP protein levels through western blotting. Primary rabbit antibodies against survivin, XIAP, cIAP1, cIAP2, livin, p53 and GAPDH were purchased from Cell Signaling Technology, Inc. (Danvers, MA) and used according to manufacture instructions as reported previously (273). Protein lane intensities were quantified by ImageJ software (US National Institutes of Health, Bethesda, MD).

\subsubsection{DARTS Assay}

Drug affinity responsive target stability (DARTS) assay was performed to identify the protein targets of UC-112 analogs in A375 or M14 cell lysates following the protocols described in Dr. Jing Huang's publications $(275,276)$. In brief, A375 or M14 lysates were prepared in nondenaturing M-PER lysis buffer (Thermo Fisher Scientific, Waltham, MA) with protease and phosphatase inhibitors. Then TNC (Tris, $\mathrm{NaCl}, \mathrm{CaCl}_{2}$ ) buffer was added into cell lysates before the total protein concentration of lysate being determined by BCA protein assay kit (Thermo Fisher Scientific, Waltham, MA). Next the lysates were split into two groups: one group was added with compound of interest solution, the other group was added with same amount of vehicle (DMSO). The samples were mixed thoroughly and incubated at room temperature for $1 \mathrm{~h}$. Then $10 \mathrm{mg} / \mathrm{mL}$ pronase stock solution was added into both compound treated or vehicle control groups to achieve the final dilution of 1:100,1:300, 1:1000 and 1:3000. One aliquot of each group was kept as un-digested control. The proteolysis was performed at room temperature for $30 \mathrm{~min}$ before ice cold protease inhibitor stock being added into the mixture. Then SDS loading buffer was added into all the samples and heated to $70{ }^{\circ} \mathrm{C}$ for $10 \mathrm{~min}$. Finally, the results were analyzed by SDS-PAGE and immunoblotting.

\subsubsection{Molecular Modeling}

The molecular docking studies were performed following similar procedure as described before $(273,277)$ in Schrodinger Molecular Modeling Suite 2014 (Schrodinger Inc., Portland, OR). All the ligands were prepared to generate various conformation before being docked into the Smac AVPI binding site of a human survivin crystal structure (Protein Data Bank entry: 3UIH). Molecular dynamic calculation was done after the docking to minimize the energy of potential ligand binding poses. Results were visualized using the Maestro interface of the Schrodinger software. 


\subsubsection{Liver Microsomal Stability Study}

Liver microsomal metabolic stability was conducted by incubating the compounds of interest $(0.5 \mu \mathrm{M})$ in a total reaction volume of $1 \mathrm{~mL}$ containing $1 \mathrm{mg} / \mathrm{mL}$ microsomal in reaction buffer [0.2 $\mathrm{M}$ of phosphate buffer solution ( $\mathrm{pH} 7.4), 1.3 \mathrm{mM}$ nicotinamide adenine dinucleotide phosphate (NADP+), $3.3 \mathrm{mM}$ glucose-6-phosphate, and $0.4 \mathrm{U} / \mathrm{mL}$ glucose-6-phosphate dehydrogenase] shaking at $37^{\circ} \mathrm{C} 100 \mathrm{rpm}$. The NADPH regenerating system (solution $\mathrm{A}$ and $\mathrm{B}$ ) was obtained from $\mathrm{BD}$ Biosciences (Bedford, MA). Aliquots $(100 \mu \mathrm{L})$ from the reaction mixtures were sampled at $0,5,10$, $20,30,60,90$ and $120 \mathrm{~min}$. Ice cold acetonitrile $(150 \mu \mathrm{L})$ containing $200 \mathrm{nM}$ of the internal standard was added to quench the reaction and to precipitate the proteins. Samples operated under similar condition using heat-denatured microsomal were used as controls. After centrifugation at 13,000g for $15 \mathrm{~min}$, the supernatant was submitted for LC-MS/MS analysis.

\subsubsection{Plasma Protein Bounding Study}

Plasma (human and rat) protein binding was analyzed using a rapid equilibrium dialysis (RED) device (Thermo Fisher Scientific, Waltham, MA) containing dialysis membrane with molecular weight cut-off at 8,000 Daltons. $50 \mu \mathrm{M}$ test compound was sparkled into the plasma chamber. Then dialysis was performed at $37^{\circ} \mathrm{C}$ with $100 \mathrm{rpm}$ shaking for $4 \mathrm{~h}$ as per manufacturer's recommendation. At the end of dialysis, $50 \mu \mathrm{L}$ aliquot from both plasma chamber and buffer saline chamber was removed and quenched with equal volume of ice cold acetonitrile with internal standard in $-20{ }^{\circ} \mathrm{C}$ for $20 \mathrm{~min}$. After centrifugation at 13,000g for 15 min, the supernatant was submitted for LC-MS/MS analysis. Free drug percentage was calculated as peak area of test compound in dialysate buffer divided by peak area of test compound in dialysate plasma.

\subsubsection{In Vivo Anti-tumor Efficacy Test}

$3 \times 10^{6}$ A375 cells were implanted into the left-side dorsal flank of each nude mouse $(n=5)$ to establish the human melanoma A375 tumor xenograft model as described previously $(129,273)$. Compound MX106 were first dissolved in DMSO then diluted by $2 \%$ methylcellulose PBS buffer. The proportion of DMSO in final solution was kept at lower than 5\%. Mice body weight and the size of tumor were closely monitored during the 3 week continuous treatment (i.p. injection, one dose per day five days per week). At the end point of the treatment, mice were sacrificed after anesthesia.

\subsubsection{TUNEL Assay}

A375 tumor tissues collected from the in vivo efficacy study in the above were fixed in formalin phosphate buffer for one week. Then the tissues were processed to get paraffin embedded sections. TUNEL assay was performed using DeadEnd ${ }^{\mathrm{TM}}$ 
Fluorometric kit (Promega Corporation, Madison, WI) following manufacturer's instructions. By the end of the experiment, VECTASHIELD ${ }^{\circledR}$ Hard Set $^{\mathrm{TM}}$ mounting medium with DAPI (Vector Lab, Inc., Burlingame, CA) was used to mount the tumor slides and stain the nuclei. The final slides were analyzed immediately under a fluorescence microscope $\left(\operatorname{EVOS}^{\circledR}\right.$ FL Cell Imaging System, Thermo Fisher Scientific Inc., NY).

\subsection{Results and Discussion}

\subsubsection{UC-112 Analogs Could Overcome Multidrug Resistance}

The co-existing of survivin and drug resistant marker in tumor tissue has hindered the in vivo anti-cancer activity of survivin inhibitors. In order to determine whether the new analogs we made can overcome multidrug resistance (MDR), we compared the activity of those analogs against multidrug-resistant melanoma cells (M14/LCCMDR1) and their parental sensitive cancer cells (M14). This pair of cell line is well validated and widely used to assess whether the drugs can overcome Pgp-mediated MDR (278-280). Our three best compounds MX86, MX35 and MX106 together with the most successful small molecule survivin inhibitor YM155 were tested on both the MDR melanoma cells and their parental cells (Table 5-1). The resistance index is calculated by dividing the $\mathrm{IC}_{50}$ value of the resistant cell line by $\mathrm{IC}_{50}$ value of the sensitive parental cell line. So the smaller this value, the better resistance overcoming effect obtained. As this table shows, compounds MX86, MX35 and MX106 all have very small resistance indexes $(1.8,1.9$ and 2.3 respectively). Their activity in the resistant cell line is comparable with that in the parental cell line. For existing survivin inhibitor, YM155, although it is very potent in parental cell line $\left(\mathrm{IC}_{50}\right.$ at $3 \mathrm{nM}$ ), its activity in resistant cell line is considerably lower $\left(\mathrm{IC}_{50}\right.$ is higher than $10 \mu \mathrm{M}$, resistance index is higher than 2941). This data indicate that the new UC-112 analogs can circumvent Pgp-mediated multi-drug resistance and are distinct from that of YM-155.

\subsubsection{UC-112 Analogs Showed Good Anti-proliferation Effects with Selectivity in NCI-60 Cell Line Screening}

UC-112 and three potent analogs MX86, MX106 and MX35 were submitted to $\mathrm{NCI}$ for its one-concentration $(10 \mu \mathrm{M})$ screening against the NCI-60 cell lines. All four compounds showed good activity and were selected for the subsequent five doses testing to determine their growth inhibition potency $\left(\mathrm{GI}_{50}\right)$ in the NCI-60 cell lines. As shown in Figure 5-1, structure modification from UC-112 to our best compound MX106 has improved the average $\mathrm{GI}_{50}$ by nearly four times $(2.2 \mu \mathrm{M}$ for UC-112 vs. $0.5 \mu \mathrm{M}$ for MX106).

The heat map in Figure 5-2 summarizes the compound growth inhibition pattern which is characterized by the $\mathrm{GI}_{50}$ mean values from NCI-60 screening. UC-112 and its 
Table 5-1. In vitro anti-proliferation activities of UC-112 analogs determined by MTS assay $(n=4)$

$$
\mathrm{IC}_{50} \pm \operatorname{SEM}(\mu \mathrm{M})
$$

\begin{tabular}{|c|c|c|c|c|c|c|c|c|}
\hline Structure & ID & $\mathbf{X}$ & $\mathbf{R}^{1}$ & A375 & PC-3 & M14 & $\begin{array}{c}\text { M14/LCC } \\
\text { 6MDR1 }\end{array}$ & $\begin{array}{l}\text { Resistance } \\
\text { Index }\end{array}$ \\
\hline \multirow{5}{*}{ (c) } & UC-112 & $\mathrm{O}$ & $\mathrm{H}$ & $\begin{array}{c}1.9 \pm \\
0.6\end{array}$ & $\begin{array}{c}1.6 \pm \\
1.0\end{array}$ & $\begin{array}{c}2.1 \pm \\
0.3\end{array}$ & $3.2 \pm 0.5$ & 1.5 \\
\hline & MX35 & $\mathrm{S}$ & $\mathrm{H}$ & $\begin{array}{c}1.4 \pm \\
0.3\end{array}$ & $\begin{array}{c}1.1 \pm \\
0.1\end{array}$ & $\begin{array}{c}1.4 \pm \\
0.2\end{array}$ & $2.9 \pm 0.6$ & 2.1 \\
\hline & MX86 & $\mathrm{O}$ & $\mathrm{Br}$ & $\begin{array}{c}0.9 \pm \\
0.2\end{array}$ & $\begin{array}{c}0.7 \pm \\
0.3\end{array}$ & $\begin{array}{c}1.0 \pm \\
0.2\end{array}$ & $1.8 \pm 0.6$ & 1.8 \\
\hline & MX106 & $\mathrm{O}$ & $\begin{array}{l}\text { i- } \\
\operatorname{Pr}\end{array}$ & $\begin{array}{c}0.9 \pm \\
0.1\end{array}$ & $\begin{array}{c}0.7 \pm \\
0.1\end{array}$ & $\begin{array}{c}0.8 \pm \\
0.2\end{array}$ & $1.8 \pm 0.4$ & 2.3 \\
\hline & YM155 & & & $\begin{array}{c}0.003 \\
\pm \\
0.002\end{array}$ & $\begin{array}{c}0.005 \\
\pm \\
0.002\end{array}$ & $\begin{array}{c}0.003 \\
\pm \\
0.001\end{array}$ & $>10$ & $>2941$ \\
\hline
\end{tabular}

Resistance Index was calculated through dividing $\mathrm{IC}_{50}$ value in resistant cell line (M14/LCC6MDR1) by $\mathrm{IC}_{50}$ in sensitive cell line (M14) for each compound. 


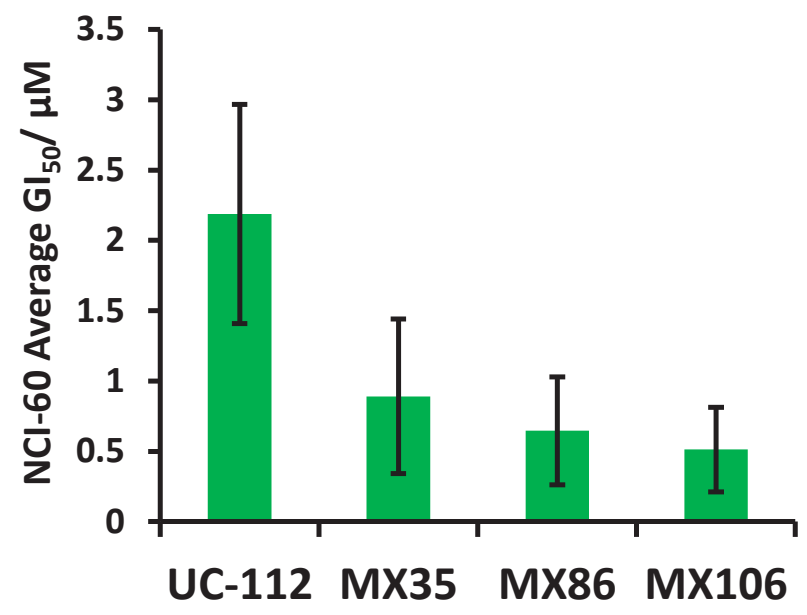

Figure 5-1. Average GI 50 $_{0}$ data for UC-112, compound MX35, MX86 and MX106 tested in NCI-60 anti-proliferative screening

Data is shown with mean $\pm \mathrm{SD}$ as bar graph. 
new analogs showed interesting selective growth inhibition behavior within the NCI 60 cell lines. The $\mathrm{GI}_{50}$ value of compound MX106 in renal cancer cell line UO-31 was as low as $52.5 \mathrm{nM}$. Interestingly, two other cell lines, HCT-15 (colon cancer) and $\mathrm{NCI} / \mathrm{ADR}-\mathrm{RES}$ (ovarian cancer), were also particularly sensitive to the treatment of UC-112 and its analogs, with lowest GI $_{50}$ value (highest activity) for compound MX106 at $46.8 \mathrm{nM}$ and $50.1 \mathrm{nM}$, respectively. Since colorectal adenocarcinoma HCT-15 cells intrinsically expresses moderate levels of Pgp, multidrug-resistance-associated protein (MRP) and lung-resistance-associated protein (LRP) (281), and ovarian cancer NCI/ADR-RES cells are naturally over-expressing MDR1 and resistant to various chemotherapies including doxorubicin (282), these data have supported our findings that UC-112 and its analogs could effectively overcome the multidrug resistance in vitro.

\subsubsection{Mechanism of Action Studies}

In our recently report (273), UC-112 exerted its tumor killing effect by selectively inhibiting the expression of survivin. In order to determine whether the new UC-112 analogs maintained the same mechanism of action, we performed the western blotting assay for MX106 in A375 and PC-3 cells (Figure 5-3A). Compound MX106 dosedependently suppressed survivin level in these two cancer cell lines, while the levels of other IAPs were minimally affected. The presence of compound MX106 at $300 \mathrm{nM}$ for $24 \mathrm{~h}$ reduced survivin levels over 50\% in both A375 and PC-3 cells (lane density was quantified by ImageJ software, data not shown). Interestingly, A375 is a p53 wild type cancer cell, whereas PC-3 is a p53 null cancer cell. As shown in Figure 5-3B, UC-112 and MX106 reduced the level of survivin in both A375 and PC-3 cells, which indicated the survivin down-regulation effects of UC-112 and MX106 is not p53 status dependent.

Next, we have conducted DARTS assay which is designed on the fact that the protease susceptibility of the target protein will decrease upon ligand binding. DARTS methodology is developed for general identifying protein-ligand interactions and has been proved to be universally applicable without acquiring the modification of ligand or targeted protein $(275,276)$. A375 and M14 cell lysates were pre-incubated with $1 \mu \mathrm{M}$ MX106 before proteolysis with pronase at gradient concentrations. Immunoblotting results in Figure 5-4 revealed that incubation with MX106 protected the survivin protein from the digestion of pronase, comparing with untreated samples. Non-target proteins like GAPDH were non-differentiated digested by pronase with or without the presence of MX106. Furthermore, pronase digestion of XIAP, cIAP1 and cIAP2 proteins was not protected by the pre-incubation of MX106 in our study. The observation indicated the existing of some specific interaction between MX106 and survivin protein, which made the later more resistant to pronase digestion in A375 and M14 cell lysates.

\subsubsection{Molecular Modeling Study}

To understand the observed improvement in potency of compound MX106 over its parental compound UC-112, we developed a molecular model and performed the 


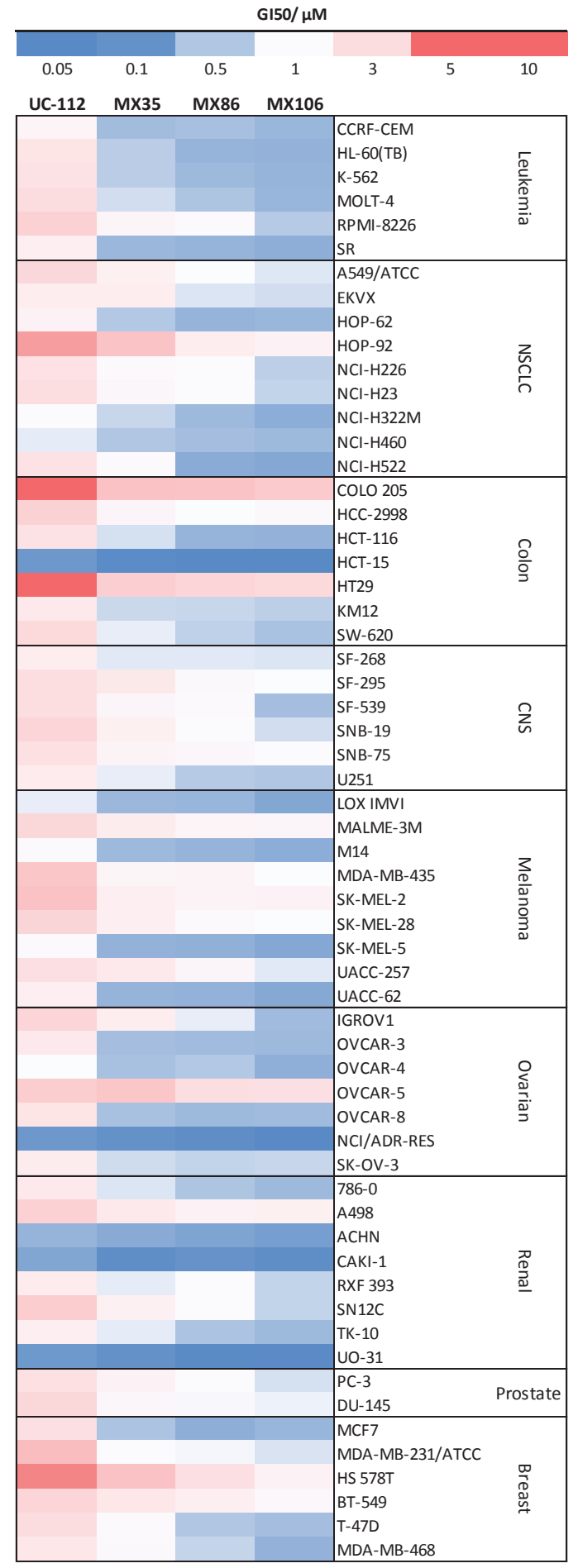

Figure 5-2. Heat map showing the $\mathrm{GI}_{50}$ values $(\mathrm{nM})$ for $\mathrm{UC}-112$ and three analogs in the NCI-60 screening

High intensity (blue) cells indicate high activity and low intensity (red) cells indicate low activity. Average $\mathrm{GI}_{50}$ values were calculated for each compound, separately. 


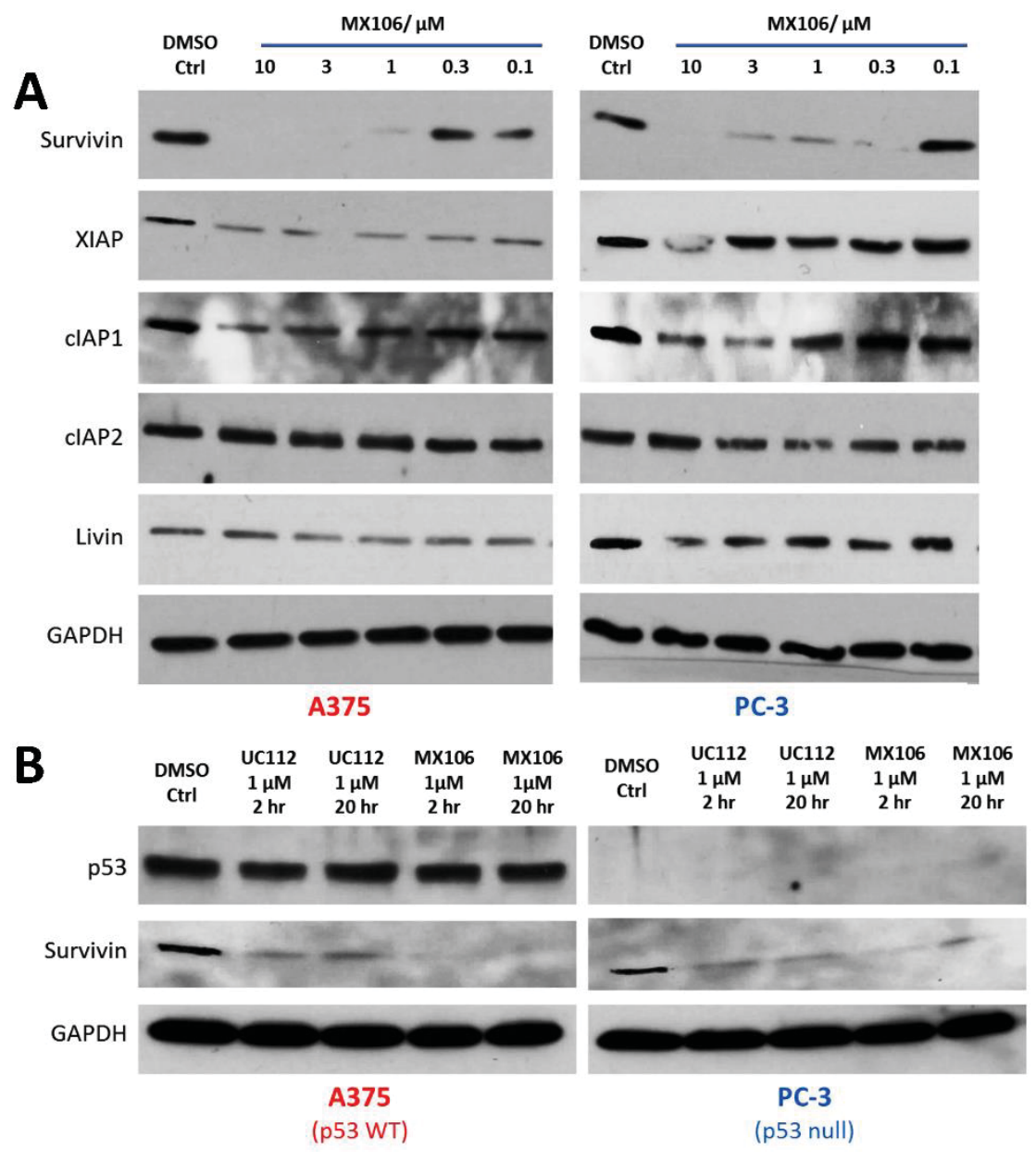

Figure 5-3. Western blotting analysis of MX106 in A375 and PC-3 cell lines A, representative images of western blotting analysis of A375 or PC-3 cells treated with gradient increasing concentrations of MX106 for $24 \mathrm{~h}$. B, representative images of western blotting analysis of A375 or PC-3 cells treated with $1 \mu \mathrm{M}$ UC-112 or MX106 for $2 \mathrm{~h}$ or $20 \mathrm{~h}$. 


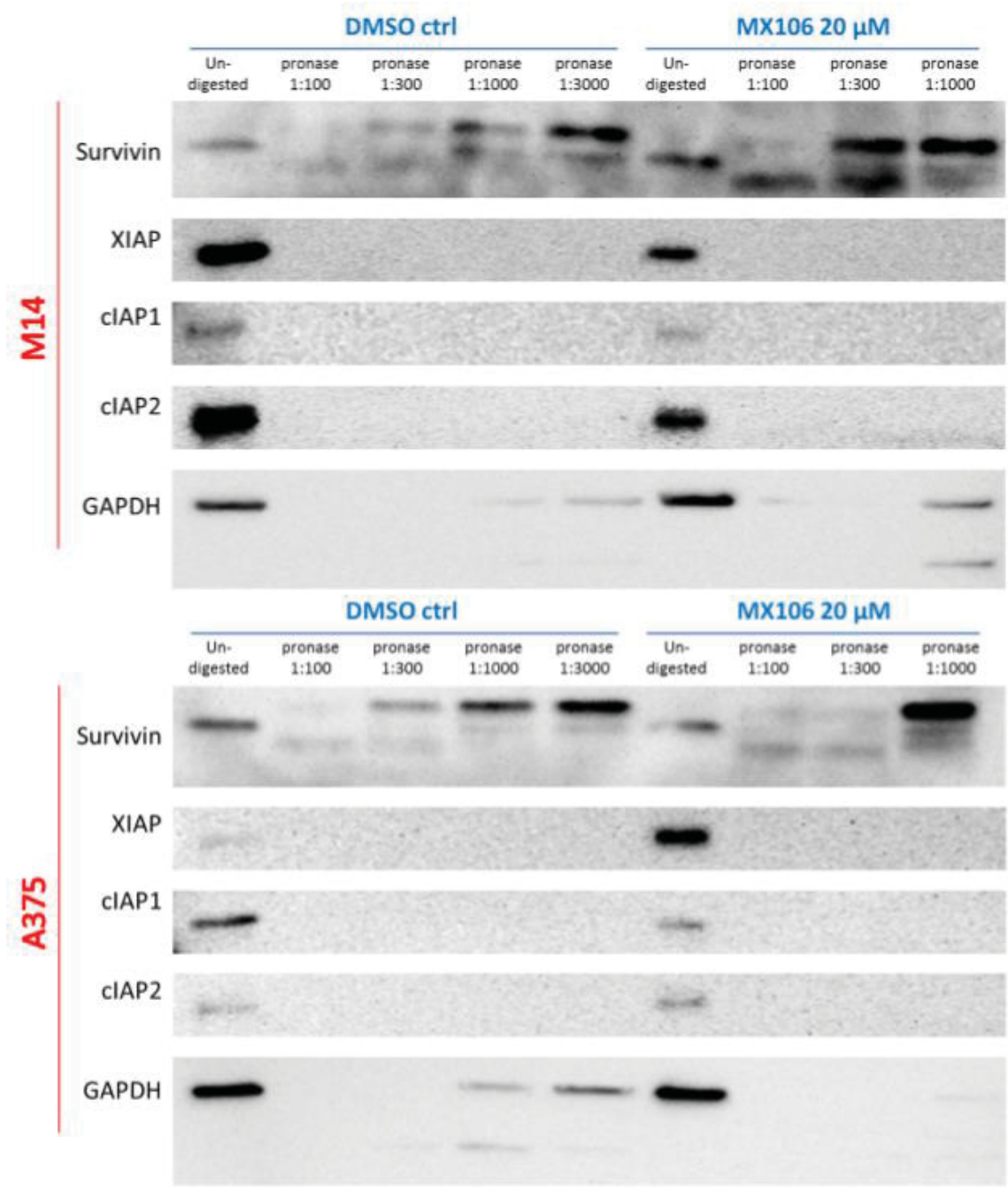

Figure 5-4. Representative DARTS results for pronase-digested A375 or M14 cell lysates

Immunoblotting showed protection of the target protein, survivin, by the incubation with MX106, whereas digestion of the non-target proteins like GAPDH was unchanged. 
molecular docking study using the complex of human survivin-Smac AVPI (PDB entry: 3UIH). As shown in Figure 5-5A, UC-112 displayed several interesting interactions with the survivin protein BIR domain: (1) two hydrogen bonding interactions between the A/B-ring of UC-112 and residue Asp71; (2) one hydrogen bonding interaction between the D-ring nitrogen and residue Glu68; and (3) an $\pi-\pi$ stacking interaction between the A/B-ring of UC-112 and residue Typ67 (Figure 5-5A). Examination of this proposed binding pose clearly suggests that the un-substituted phenyl ring of UC-112 failed to occupy a hydrophobic groove (cycled with green dash line in Figure 5-5A), and a properly sized, non-polar para-substitution (e.g., an isopropyl moiety as in compound MX106) to this ring would fill this groove and also provide excellent overlap with the bioactive AVPI peptide (Figure 5-5B). The binding mode is consistent with our experimental results. Further refinement to this model, and ultimately an X-ray crystal structure will greatly facilitate the future optimization of this scaffold.

\subsubsection{In Vivo Anti-tumor Efficacy Assessment}

Since our in vitro study showed that compound MX106 has the highest antiproliferative potency in this series of UC-112 analogs, we selected MX106 to test its in vivo efficacy against tumor growth in a human melanoma A375 xenograft model through i.p injection. As shown in the Figure 5-6A and B, compound MX106 inhibited the growth of A375 xenograft tumor in a dose-dependent manner during the three weeks of continuous treatment group is $53 \%$ and $79 \%$ slower than the vehicle control group, respectively. Terminal deoxynucleotidyl transferase dUTP nick end labeling (TUNEL) assay, which measured the nuclear DNA fragmentation, showed that a high dose (40 $\mathrm{mg} / \mathrm{kg}$ ) of compound MX106 treatment caused the widely-spread cell apoptosis inside the tumor tissues (Figure 5-6C). 

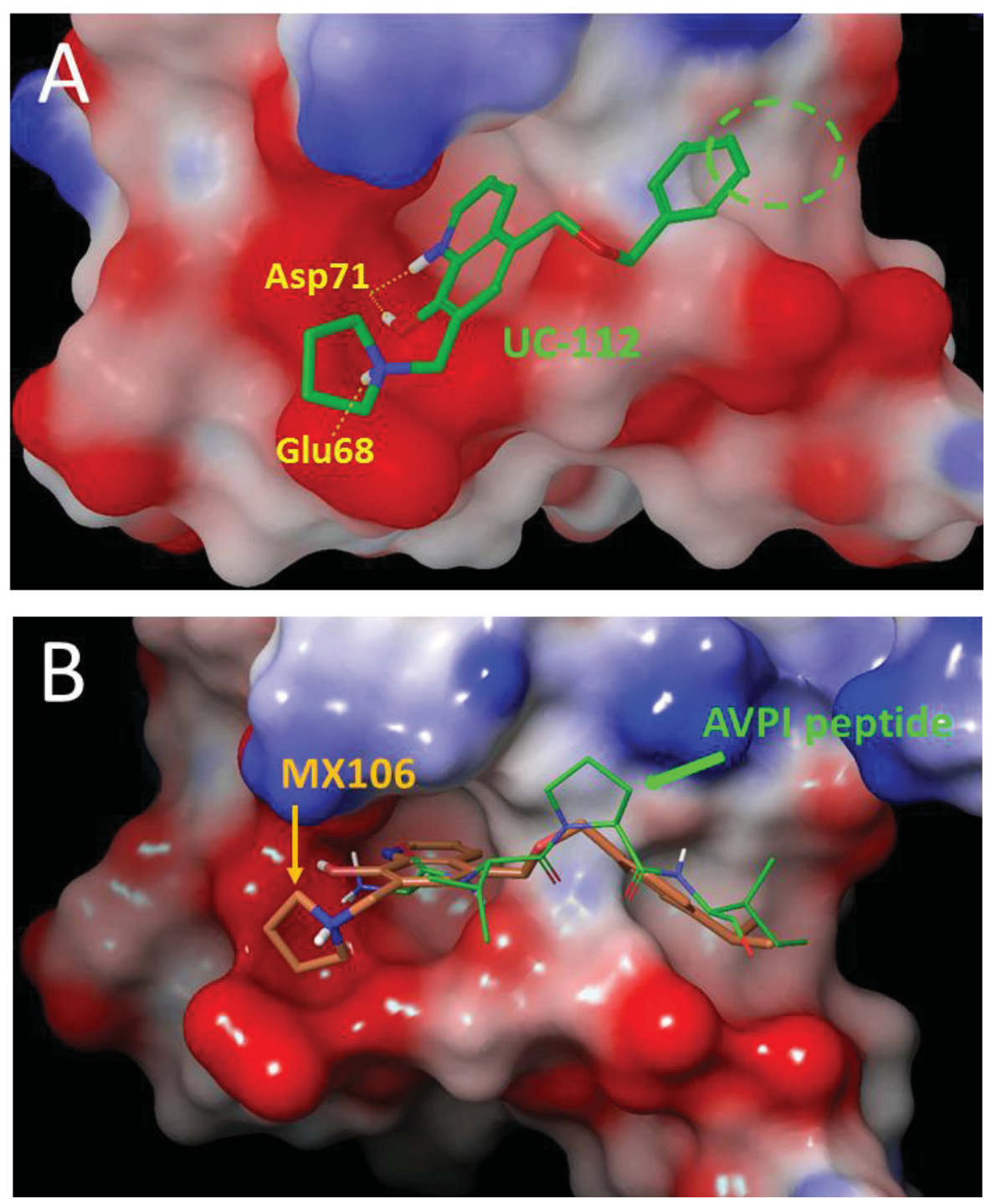

Figure 5-5. Potential binding pose of UC-112 and compound MX106 in the Smac N-terminus tetra-peptide AVPI binding site of survivin crystal structure (PDB entry: 3UIH) .

The surface of AVPI binding site in survivin was colored according to residue charge (blue for positive while red for negative). A, UC-112 (green tube) formed hydrogen bonds with residue Asp71 and Glu68 on the survivin protein BIR domain. B, compound MX106 (orange tube) displayed similar binding pose with UC-112 but had better occupied the grove toward the N-terminus of survivin protein. And this pose was overlapping well with the binding mode of native ligand, Smac AVPI (green stick). 


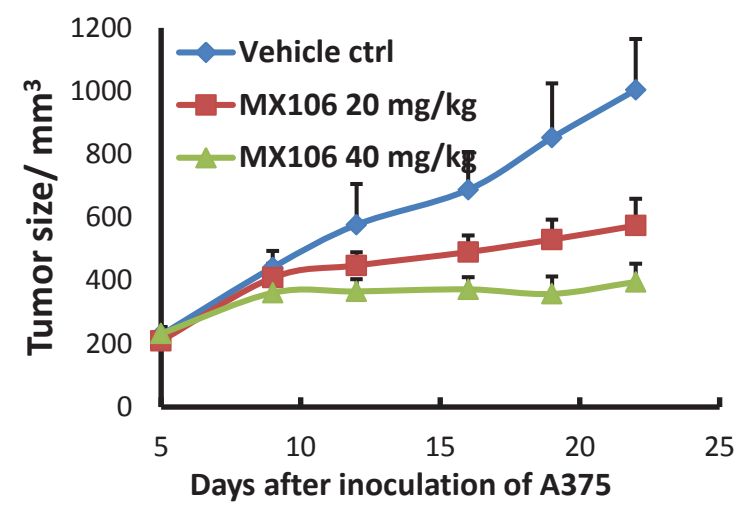

DAPI
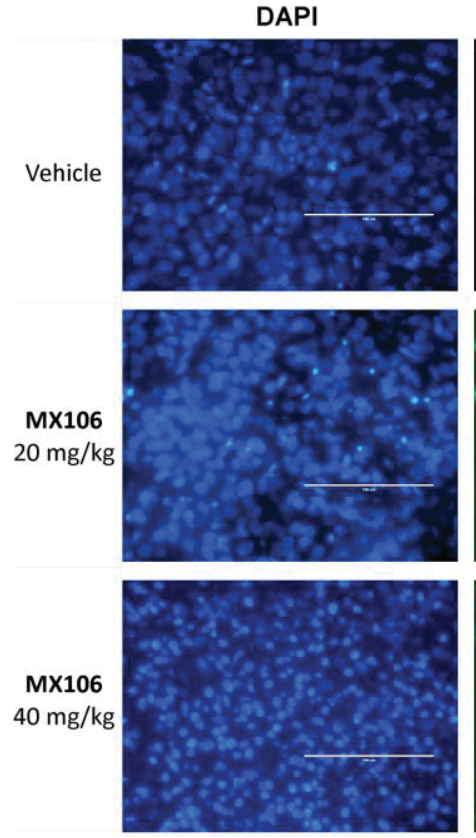

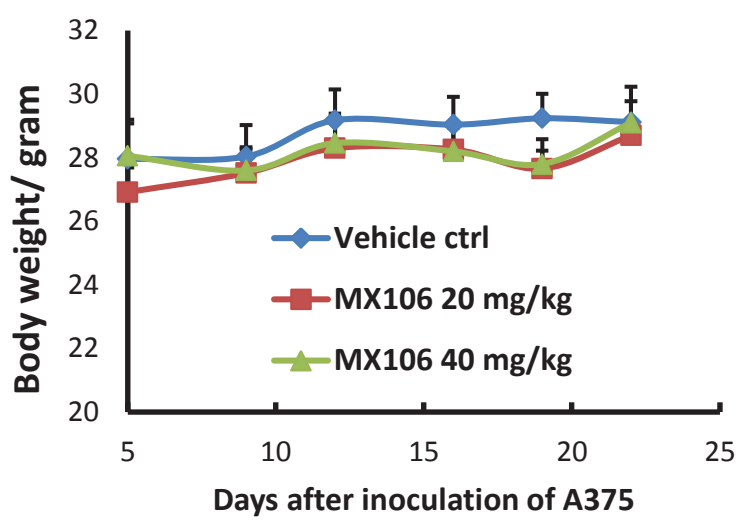

GFP
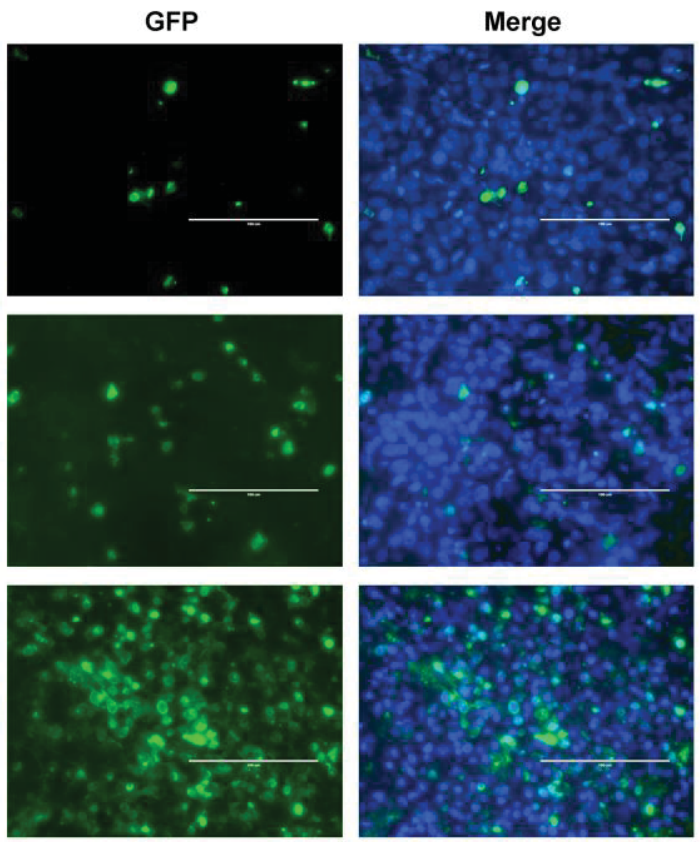
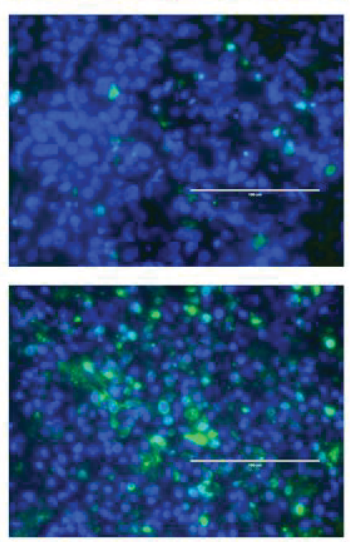

Figure 5-6. In vivo anti-tumor efficacy of compound MX106

A and B, MX106 effectively inhibited the growth of A375 xenograft tumor after three weeks continuous treatment (i.p. injection) in a dose-dependent manner (A) without causing obvious decrease of mice body weight (B). C, Representative images of TUNEL assay using the formalin-fixed tumor sections. 


\section{CHAPTER 6. SUMMARY AND FUTURE DIRECTION}

In the combination project, since one of the major hallmarks for tubulin inhibitors is their ability to strongly arrest cells in the $\mathrm{G}_{2} / \mathrm{M}$ phase, we hypothesized the combination of vemurafenib and a tubulin inhibitor would synergistically arrest melanoma cells, leading to enhanced apoptosis, and overcome acquired resistance. In this study we selected novel tubulin inhibitors, ABI-231 and ABI-274, to investigate its combination with vemurafenib against melanoma tumors. We developed vemurafenibresistant human melanoma cell line A375RF21 and MDA-MB-435 VemR. Results showed that the combination of ABI compound and vemurafenib had strong synergy in vitro. We confirmed that the synergy is unlikely through enhanced inhibition of tubulin polymerization or diminished of p-ERK activation. Instead, our experimental results revealed that this combined treatment overcomes the acquired vemurafenib-resistance through enhanced apoptosis induction produced by synergistic $G_{1}$ and $G_{2} / M$ cell-cycle arrest and substantially impaired the survival signaling pathway related to AKT phosphorylation. We showed that the strong synergy observed in vitro clearly translated to significant efficacy in vivo when tested in a vemurafenib-resistant xenograft model. Further immunohistochemistry analyses on tissue sections confirmed the strong inhibition of tumor proliferation and the diminished activity of pAKT. The in vivo studies presented here show an effective combination treatment in tumor cells that are already vemurafenib-resistant by using A375RF21 xenograft models. It is conceivable that if we used the combination before tumor cells became resistant to vemurafenib, tumor regression may be more enhanced and we could significantly delay or even prevent the development of resistant tumor cells. This could translate into at least a substantially longer progression-free time in patients, and/or enhanced disease regression. Collectively, our study offers the first direct evidence and a rationale for combining a potent tubulin inhibitor with an inhibitor targeting the RAF/MEK/ERK pathway for greatly improved therapy for melanoma patients. To further conduct this project, we need to address three types of questions: 1) can we find a better methodology other than CI values to precisely evaluate the combination effect test in vitro; 2) can we establish more BRAF inhibition resistant models with known and clinical relevant mechanisms; 3) can we deal with the tumor heterogeneity, which is extremely important in control of acquired resistance to small molecule cancer therapy, through rational designed combination treatment.

In the survivin inhibitor project, using shape-based virtual screening combined with biological activity evaluation, we identified four Smac mimetic hits with higher or comparable anti-proliferation potency compared with embelin. The overall hypothesis is, theses hit compounds can mimic the function of Smac, which is the endogenous inhibitor of IAPs including survivin, and thus trigger the programmed apoptosis in cancer cells. The four identified hits significantly activated caspase-3/7 and caspase- 9 function in human melanoma A375 and prostate cancer PC-3 cell lines. Compounds UC-112 and UC-222 strongly suppressed XIAP and survivin levels in vitro and had scaffolds amicable for modification to further improve their potency. In particular, compound UC112 showed good selectivity in decreasing the level of survivin (BIRC5) in a dosedependent manner, possibly through the ubiquitin-mediated degradation pathways, and it also has the potential to overcome Pgp-mediated multidrug-resistance which is a 
limitation for many of the existing IAP inhibitors. More excitingly, UC-112 displayed very low toxicity, showed potent efficacy of tumor growth inhibition in an A375 melanoma xenograft model, and maintained its mechanisms of actions in vivo. This integrated chemical biology study provided promising lead compounds amicable for further structural optimization in order to develop new selective IAP inhibitors as potential therapeutic agents for resistant cancers, either as a single agent or in combinations with existing drugs. Novel analogs which are structurally modified from the lead compound UC-112 have showed excellent anti-proliferative activities and could also effectively overcome Pgp-mediated multiple drug resistance. The most potent compound MX106 has selectively down-regulated the level of survivin as the parent compound UC-112 in a p53 status independent manner. And compound MX106 greatly inhibited the growth of A 375 xenograft tumor in vivo. These findings are encouraging but target validation work is essential to further direct the future study of this project. In other words, we need to know why the level of survivin protein is decreased after the treatment of UC-112 and its analogs. Considering the regulation mechanisms of survivin, we should investigate whether the compounds are promoting degradation or inhibiting the expression of survivin. Meanwhile, the off-target effect of this series of compounds need to be properly evaluate although in the xenograft models, i.p. injection for three continued weeks at doses up to $40 \mathrm{mg} / \mathrm{kg}$ did not significantly affect the nude mice body weight. 


\section{LIST OF REFERENCES}

1. Hersh EM, O'Day SJ, Powderly J, Khan KD, Pavlick AC, Cranmer LD, et al. A phase II multicenter study of ipilimumab with or without dacarbazine in chemotherapy-naive patients with advanced melanoma. Invest New Drugs. 2011;29: 489-498.

2. Sosman JA, Kim KB, Schuchter L, Gonzalez R, Pavlick AC, Weber JS, et al. Survival in BRAF V600-mutant advanced melanoma treated with vemurafenib. N Engl J Med. 2012;366: 707-714.

3. Al-Lazikani B, Workman P. Unpicking the combination lock for mutant BRAF and RAS melanomas. Cancer Discov. 2013;3: 14-19.

4. Hu-Lieskovan S, Robert L, Homet Moreno B, Ribas A. Combining Targeted Therapy With Immunotherapy in BRAF-Mutant Melanoma: Promise and Challenges. J Clin Oncol. 2014;32: 2248-2254.

5. Nijenhuis CM, Haanen JB, Schellens JH, Beijnen JH. Is combination therapy the next step to overcome resistance and reduce toxicities in melanoma? Cancer Treat Rev. 2013;39: 305-312.

6. Voskoboynik M, Arkenau HT. Combination therapies for the treatment of advanced melanoma: a review of current evidence. Biochem Res Int. 2014;2014: 307059.

7. Wang J, Miller DD, Li W. Emerging Drug Combination Approaches in Melanoma Therapy. InTech, 2015. ISBN: 978-953-51-2036-0, DOI: $10.5772 / 59360$

8. Wan PT, Garnett MJ, Roe SM, Lee S, Niculescu-Duvaz D, Good VM, et al. Mechanism of activation of the RAF-ERK signaling pathway by oncogenic mutations of B-RAF. Cell. 2004;116: 855-867.

9. Tsai J, Lee JT, Wang W, Zhang J, Cho H, Mamo S, et al. Discovery of a selective inhibitor of oncogenic B-Raf kinase with potent antimelanoma activity. Proc Natl Acad Sci U S A. 2008;105: 3041-3046.

10. King AJ, Arnone MR, Bleam MR, Moss KG, Yang J, Fedorowicz KE, et al. Dabrafenib; preclinical characterization, increased efficacy when combined with trametinib, while BRAF/MEK tool combination reduced skin lesions. PLoS One. 2013;8: e67583.

11. Solit DB, Rosen N. Towards a unified model of RAF inhibitor resistance. Cancer Discov. 2014;4: 27-30.

12. Hooijkaas A, Gadiot J, Morrow M, Stewart R, Schumacher T, Blank CU. Selective BRAF inhibition decreases tumor-resident lymphocyte frequencies in a mouse model of human melanoma. Oncoimmunology. 2012;1: 609-617.

13. Menzies AM, Long GV. Dabrafenib and trametinib, alone and in combination for BRAF-mutant metastatic melanoma. Clin Cancer Res. 2014;20: 2035-2043.

14. Carlino MS, Gowrishankar K, Saunders CA, Pupo GM, Snoyman S, Zhang XD, et al. Antiproliferative effects of continued mitogen-activated protein kinase pathway inhibition following acquired resistance to BRAF and/or MEK inhibition in melanoma. Mol Cancer Ther. 2013;12: 1332-1342. 
15. Klein O, Clements A, Menzies AM, O'Toole S, Kefford RF, Long GV. BRAF inhibitor activity in V600R metastatic melanoma. European Journal of Cancer. 2013;49: 1073-1079.

16. Vin H, Ojeda SS, Ching G, Leung ML, Chitsazzadeh V, Dwyer DW, et al. BRAF inhibitors suppress apoptosis through off-target inhibition of JNK signaling. Elife. 2013;2: e00969.

17. Roesch A. Tumor heterogeneity and plasticity as elusive drivers for resistance to MAPK pathway inhibition in melanoma. Oncogene. 2014.

18. Yancovitz M, Litterman A, Yoon J, Ng E, Shapiro RL, Berman RS, et al. Intraand inter-tumor heterogeneity of $\mathrm{BRAF}(\mathrm{V} 600 \mathrm{E}))$ mutations in primary and metastatic melanoma. PLoS One. 2012;7: e29336.

19. Little AS, Smith PD, Cook SJ. Mechanisms of acquired resistance to ERK1/2 pathway inhibitors. Oncogene. 2013;32: 1207-1215.

20. Tentori L, Lacal PM, Graziani G. Challenging resistance mechanisms to therapies for metastatic melanoma. Trends Pharmacol Sci. 2013;34: 656-666.

21. Straussman R, Morikawa T, Shee K, Barzily-Rokni M, Qian ZR, Du J, et al. Tumour micro-environment elicits innate resistance to RAF inhibitors through HGF secretion. Nature. 2012;487: 500-504.

22. Johannessen CM, Boehm JS, Kim SY, Thomas SR, Wardwell L, Johnson LA, et al. COT drives resistance to RAF inhibition through MAP kinase pathway reactivation. Nature. 2010;468: 968-972.

23. Wagle N, Emery C, Berger MF, Davis MJ, Sawyer A, Pochanard P, et al. Dissecting therapeutic resistance to RAF inhibition in melanoma by tumor genomic profiling. J Clin Oncol. 2011;29: 3085-3096.

24. Poulikakos PI, Zhang C, Bollag G, Shokat KM, Rosen N. RAF inhibitors transactivate RAF dimers and ERK signalling in cells with wild-type BRAF. Nature. 2010;464: 427-430.

25. Gowrishankar K, Snoyman S, Pupo GM, Becker TM, Kefford RF, Rizos H. Acquired resistance to BRAF inhibition can confer cross-resistance to combined BRAF/MEK inhibition. J Invest Dermatol. 2012;132: 1850-1859.

26. Carlino MS, Todd JR, Gowrishankar K, Mijatov B, Pupo GM, Fung C, et al. Differential activity of MEK and ERK inhibitors in BRAF inhibitor resistant melanoma. Mol Oncol. 2014;8: 544-554.

27. Sanchez-Laorden B, Viros A, Girotti MR, Pedersen M, Saturno G, Zambon A, et al. BRAF inhibitors induce metastasis in RAS mutant or inhibitor-resistant melanoma cells by reactivating MEK and ERK signaling. Sci Signal. 2014;7: $\mathrm{ra30.}$

28. Su F, Bradley WD, Wang Q, Yang H, Xu L, Higgins B, et al. Resistance to selective BRAF inhibition can be mediated by modest upstream pathway activation. Cancer Res. 2012;72: 969-978.

29. Nathanson KL, Martin AM, Wubbenhorst B, Greshock J, Letrero R, D'Andrea K, et al. Tumor genetic analyses of patients with metastatic melanoma treated with the BRAF inhibitor dabrafenib (GSK2118436). Clin Cancer Res. 2013;19: 48684878. 
30. Smalley KS, Lioni M, Dalla Palma M, Xiao M, Desai B, Egyhazi S, et al. Increased cyclin D1 expression can mediate BRAF inhibitor resistance in BRAF V600E-mutated melanomas. Mol Cancer Ther. 2008;7: 2876-2883.

31. Choi J, Landrette SF, Wang T, Evans P, Bacchiocchi A, Bjornson R, et al. Identification of PLX4032-resistance mechanisms and implications for novel RAF inhibitors. Pigment Cell Melanoma Res. 2014;27: 253-262.

32. Wu CP, Sim HM, Huang YH, Liu YC, Hsiao SH, Cheng HW, et al. Overexpression of ATP-binding cassette transporter ABCG2 as a potential mechanism of acquired resistance to vemurafenib in BRAF(V600E) mutant cancer cells. Biochem Pharmacol. 2013;85: 325-334.

33. Flaherty KT, Robert C, Hersey P, Nathan P, Garbe C, Milhem M, et al. Improved survival with MEK inhibition in BRAF-mutated melanoma. N Engl J Med. 2012;367: 107-114.

34. Wright CJ, McCormack PL. Trametinib: first global approval. Drugs. 2013;73: 1245-1254.

35. Atefi M, von Euw E, Attar N, Ng C, Chu C, Guo D, et al. Reversing melanoma cross-resistance to BRAF and MEK inhibitors by co-targeting the AKT/mTOR pathway. PLoS One. 2011;6: e28973.

36. Kim KB, Kefford R, Pavlick AC, Infante JR, Ribas A, Sosman JA, et al. Phase II study of the MEK1/MEK2 inhibitor Trametinib in patients with metastatic BRAFmutant cutaneous melanoma previously treated with or without a BRAF inhibitor. J Clin Oncol. 2013;31: 482-489.

37. Flaherty KT, Infante JR, Daud A, Gonzalez R, Kefford RF, Sosman J, et al. Combined BRAF and MEK inhibition in melanoma with BRAF V600 mutations. N Engl J Med. 2012;367: 1694-1703.

38. Goldinger SM, Zimmer L, Schulz C, Ugurel S, Hoeller C, Kaehler KC, et al. Upstream mitogen-activated protein kinase (MAPK) pathway inhibition: MEK inhibitor followed by a BRAF inhibitor in advanced melanoma patients. European Journal of Cancer. 2014;50: 406-410.

39. Greger JG, Eastman SD, Zhang V, Bleam MR, Hughes AM, Smitheman KN, et al. Combinations of BRAF, MEK, and PI3K/mTOR inhibitors overcome acquired resistance to the BRAF inhibitor GSK2118436 dabrafenib, mediated by NRAS or MEK mutations. Mol Cancer Ther. 2012;11: 909-920.

40. Nakamura A, Arita T, Tsuchiya S, Donelan J, Chouitar J, Carideo E, et al. Antitumor activity of the selective pan-RAF inhibitor TAK-632 in BRAF inhibitor-resistant melanoma. Cancer Res. 2013;73: 7043-7055.

41. Lee CI, Menzies AM, Haydu LE, Azer M, Clements A, Kefford RF, et al. Features and management of pyrexia with combined dabrafenib and trametinib in metastatic melanoma. Melanoma Res. 2014.

42. Ribas A, Gonzalez R, Pavlick A, Hamid O, Gajewski TF, Daud A, et al. Combination of vemurafenib and cobimetinib in patients with advanced BRAF(V600)-mutated melanoma: a phase 1b study. Lancet Oncol. 2014;15: 954965. 
43. Kefford R, Miller WH, Tan DSW, Sullivan RJ, Long G, Dienstmann R, et al. Preliminary results from a phase Ib/II, open-label, dose-escalation study of the oral BRAF inhibitor LGX818 in combination with the oral MEK1/2 inhibitor MEK162 in BRAF V600-dependent advanced solid tumors. Journal of Clinical Oncology. 2013;31.

44. Dummer R, Robert C, Nyakas M, McArthur GA, Kudchadkar RR, Gomez-Roca $\mathrm{C}$, et al. Initial results from a phase I, open-label, dose escalation study of the oral BRAF inhibitor LGX818 in patients with BRAF V600 mutant advanced or metastatic melanoma. Journal of Clinical Oncology. 2013;31.

45. Carracedo A, Pandolfi PP. The PTEN-PI3K pathway: of feedbacks and crosstalks. Oncogene. 2008;27: 5527-5541.

46. Villanueva J, Vultur A, Lee JT, Somasundaram R, Fukunaga-Kalabis M, Cipolla AK, et al. Acquired resistance to BRAF inhibitors mediated by a RAF kinase switch in melanoma can be overcome by cotargeting MEK and IGF-1R/PI3K. Cancer Cell. 2010;18: 683-695.

47. Lasithiotakis KG, Sinnberg TW, Schittek B, Flaherty KT, Kulms D, Maczey E, et al. Combined inhibition of MAPK and mTOR signaling inhibits growth, induces cell death, and abrogates invasive growth of melanoma cells. J Invest Dermatol. 2008;128: 2013-2023.

48. Niessner H, Beck D, Sinnberg T, Lasithiotakis K, Maczey E, Gogel J, et al. The farnesyl transferase inhibitor lonafarnib inhibits mTOR signaling and enforces sorafenib-induced apoptosis in melanoma cells. J Invest Dermatol. 2011;131: 468-479.

49. Khalili JS, Yu X, Wang J, Hayes BC, Davies MA, Lizee G, et al. Combination small molecule MEK and PI3K inhibition enhances uveal melanoma cell death in a mutant GNAQ- and GNA11-dependent manner. Clin Cancer Res. 2012;18: 4345-4355.

50. Lassen A, Atefi M, Robert L, Wong DJ, Cerniglia M, Comin-Anduix B, et al. Effects of AKT inhibitor therapy in response and resistance to BRAF inhibition in melanoma. Mol Cancer. 2014;13: 83.

51. Byron SA, Loch DC, Wellens CL, Wortmann A, Wu J, Wang J, et al. Sensitivity to the MEK inhibitor E6201 in melanoma cells is associated with mutant BRAF and wildtype PTEN status. Mol Cancer. 2012;11: 75.

52. Posch C, Moslehi H, Feeney L, Green GA, Ebaee A, Feichtenschlager V, et al. Combined targeting of MEK and $\mathrm{PI} 3 \mathrm{~K} / \mathrm{mTOR}$ effector pathways is necessary to effectively inhibit NRAS mutant melanoma in vitro and in vivo. Proc Natl Acad Sci U S A. 2013;110: 4015-4020.

53. Shi H, Kong X, Ribas A, Lo RS. Combinatorial treatments that overcome PDGFRbeta-driven resistance of melanoma cells to V600EB-RAF inhibition. Cancer Res. 2011;71: 5067-5074.

54. Shimizu T, Tolcher AW, Papadopoulos KP, Beeram M, Rasco DW, Smith LS, et al. The clinical effect of the dual-targeting strategy involving PI3K/AKT/mTOR and RAS/MEK/ERK pathways in patients with advanced cancer. Clin Cancer Res. 2012;18: 2316-2325. 
55. Frederick DT, Piris A, Cogdill AP, Cooper ZA, Lezcano C, Ferrone CR, et al. BRAF Inhibition Is Associated with Enhanced Melanoma Antigen Expression and a More Favorable Tumor Microenvironment in Patients with Metastatic Melanoma. Clinical Cancer Research. 2013;19: 1225-1231.

56. Lev DC, Ruiz M, Mills L, McGary EC, Price JE, Bar-Eli M. Dacarbazine causes transcriptional up-regulation of interleukin 8 and vascular endothelial growth factor in melanoma cells: a possible escape mechanism from chemotherapy. Mol Cancer Ther. 2003;2: 753-763.

57. Ghosh S, Maity P. Augmented antitumor effects of combination therapy with VEGF antibody and cisplatin on murine B16F10 melanoma cells. Int Immunopharmacol. 2007; 7: 1598-1608.

58. Sini P, Samarzija I, Baffert F, Littlewood-Evans A, Schnell C, Theuer A, et al. Inhibition of multiple vascular endothelial growth factor receptors (VEGFR) blocks lymph node metastases but inhibition of VEGFR-2 is sufficient to sensitize tumor cells to platinum-based chemotherapeutics. Cancer Res. 2008;68: 15811592.

59. Terheyden P, Hofmann MA, Weininger M, Brocker EB, Becker JC. Anti-vascular endothelial growth factor antibody bevacizumab in conjunction with chemotherapy in metastasising melanoma. J Cancer Res Clin Oncol. 2007;133: 897-901.

60. Sun C, Wang L, Huang S, Heynen GJ, Prahallad A, Robert C, et al. Reversible and adaptive resistance to BRAF(V600E) inhibition in melanoma. Nature. 2014;508: 118-122.

61. Motl S. Bevacizumab in combination chemotherapy for colorectal and other cancers. Am J Health Syst Pharm. 2005;62: 1021-1032.

62. Del Vecchio M, Mortarini R, Canova S, Di Guardo L, Pimpinelli N, Sertoli MR, et al. Bevacizumab plus fotemustine as first-line treatment in metastatic melanoma patients: clinical activity and modulation of angiogenesis and lymphangiogenesis factors. Clin Cancer Res. 2010;16: 5862-5872.

63. Kottschade LA, Suman VJ, Perez DG, McWilliams RR, Kaur JS, Amatruda TT, 3 rd, et al. A randomized phase 2 study of temozolomide and bevacizumab or nabpaclitaxel, carboplatin, and bevacizumab in patients with unresectable stage IV melanoma : a North Central Cancer Treatment Group study, N0775. Cancer. 2013;119: 586-592.

64. Li B, Lalani AS, Harding TC, Luan B, Koprivnikar K, Huan Tu G, et al. Vascular endothelial growth factor blockade reduces intratumoral regulatory $\mathrm{T}$ cells and enhances the efficacy of a GM-CSF-secreting cancer immunotherapy. Clin Cancer Res. 2006;12: 6808-6816.

65. Mita MM, Rowinsky EK, Forero L, Eckhart SG, Izbicka E, Weiss GR, et al. A phase II, pharmacokinetic, and biologic study of semaxanib and thalidomide in patients with metastatic melanoma. Cancer Chemother Pharmacol. 2007;59: 165174.

66. Hasumi Y, Klosowska-Wardega A, Furuhashi M, Ostman A, Heldin CH, Hellberg C. Identification of a subset of pericytes that respond to combination therapy targeting PDGF and VEGF signaling. Int J Cancer. 2007;121: 2606-2614. 
67. O'Reilly T, Lane HA, Wood JM, Schnell C, Littlewood-Evans A, Brueggen J, et al. Everolimus and PTK/ZK show synergistic growth inhibition in the orthotopic BL16/BL6 murine melanoma model. Cancer Chemother Pharmacol. 2011;67: 193-200.

68. Hainsworth JD, Infante JR, Spigel DR, Peyton JD, Thompson DS, Lane CM, et al. Bevacizumab and everolimus in the treatment of patients with metastatic melanoma: a phase 2 trial of the Sarah Cannon Oncology Research Consortium. Cancer. 2010;116: 4122-4129.

69. Mahalingam D, Malik L, Beeram M, Rodon J, Sankhala K, Mita A, et al. Phase II study evaluating the efficacy, safety, and pharmacodynamic correlative study of dual antiangiogenic inhibition using bevacizumab in combination with sorafenib in patients with advanced malignant melanoma. Cancer Chemother Pharmacol. 2014;74: 77-84.

70. Mittal K, Koon H, Elson P, Triozzi P, Dowlati A, Chen H, et al. Dual VEGF/VEGFR inhibition in advanced solid malignancies: Clinical effects and pharmacodynamic biomarkers. Cancer Biol Ther. 2014;15: 975-981.

71. Mirmohammadsadegh A, Mota R, Gustrau A, Hassan M, Nambiar S, Marini A, et al. ERK1/2 is highly phosphorylated in melanoma metastases and protects melanoma cells from cisplatin-mediated apoptosis. J Invest Dermatol. 2007;127: 2207-2215.

72. Ivanov VN, Hei TK. Combined treatment with EGFR inhibitors and arsenite upregulated apoptosis in human EGFR-positive melanomas: a role of suppression of the PI3K-AKT pathway. Oncogene. 2005;24: 616-626.

73. Sinnberg T, Lasithiotakis K, Niessner H, Schittek B, Flaherty KT, Kulms D, et al. Inhibition of PI3K-AKT-mTOR signaling sensitizes melanoma cells to cisplatin and temozolomide. J Invest Dermatol. 2009;129: 1500-1515.

74. Keuling AM, Andrew SE, Tron VA. Inhibition of p38 MAPK enhances ABT737-induced cell death in melanoma cell lines: novel regulation of PUMA. Pigment Cell Melanoma Res. 2010;23: 430-440.

75. Frederick DT, Salas Fragomeni RA, Schalck A, Ferreiro-Neira I, Hoff T, Cooper ZA, et al. Clinical profiling of BCL-2 family members in the setting of BRAF inhibition offers a rationale for targeting de novo resistance using $\mathrm{BH} 3$ mimetics. PLoS One. 2014;9: e101286.

76. Ji Z, Kumar R, Taylor M, Rajadurai A, Marzuka-Alcala A, Chen YE, et al. Vemurafenib synergizes with nutlin-3 to deplete survivin and suppresses melanoma viability and tumor growth. Clin Cancer Res. 2013;19: 4383-4391.

77. Vultur A, Villanueva J, Krepler C, Rajan G, Chen Q, Xiao M, et al. MEK inhibition affects STAT3 signaling and invasion in human melanoma cell lines. Oncogene. 2014;33: 1850-1861.

78. Martin MJ, Hayward R, Viros A, Marais R. Metformin accelerates the growth of BRAF V600E-driven melanoma by upregulating VEGF-A. Cancer Discov. 2012;2: 344-355.

79. Woodard J, Platanias LC. AMP-activated kinase (AMPK)-generated signals in malignant melanoma cell growth and survival. Biochem Biophys Res Commun. 2010;398: 135-139. 
80. Niehr F, von Euw E, Attar N, Guo D, Matsunaga D, Sazegar H, et al. Combination therapy with vemurafenib (PLX4032/RG7204) and metformin in melanoma cell lines with distinct driver mutations. J Transl Med. 2011;9: 76.

81. Sagoo MS, Harbour JW, Stebbing J, Bowcock AM. Combined PKC and MEK inhibition for treating metastatic uveal melanoma. Oncogene. 2014.

82. Wu X, Zhu M, Fletcher JA, Giobbie-Hurder A, Hodi FS. The protein kinase C inhibitor enzastaurin exhibits antitumor activity against uveal melanoma. PLoS One. 2012;7: e29622.

83. Chen X, Wu Q, Tan L, Porter D, Jager MJ, Emery C, et al. Combined PKC and MEK inhibition in uveal melanoma with GNAQ and GNA11 mutations. Oncogene. 2013.

84. Wang J, Chen J, Miller DD, Li W. Synergistic combination of novel tubulin inhibitor ABI-274 and vemurafenib overcome vemurafenib acquired resistance in BRAFV600E melanoma. Mol Cancer Ther. 2014;13: 16-26.

85. Acquaviva J, Smith DL, Jimenez JP, Zhang C, Sequeira M, He S, et al. Overcoming acquired BRAF inhibitor resistance in melanoma via targeted inhibition of Hsp90 with ganetespib. Mol Cancer Ther. 2014;13: 353-363.

86. Yadav V, Burke TF, Huber L, Van Horn RD, Zhang Y, Buchanan SG, et al. The CDK4/6 Inhibitor LY2835219 Overcomes Vemurafenib Resistance Resulting from MAPK Reactivation and Cyclin D1 Upregulation. Mol Cancer Ther. 2014.

87. Hodi FS, O'Day SJ, McDermott DF, Weber RW, Sosman JA, Haanen JB, et al. Improved survival with ipilimumab in patients with metastatic melanoma. N Engl J Med. 2010;363: 711-723.

88. Srivastava N, McDermott D. Update on benefit of immunotherapy and targeted therapy in melanoma: the changing landscape. Cancer Manag Res. 2014;6: 279289.

89. Riley JL. Combination checkpoint blockade--taking melanoma immunotherapy to the next level. N Engl J Med. 2013;369: 187-189.

90. Maio M, Di Giacomo AM, Robert C, Eggermont AM. Update on the role of ipilimumab in melanoma and first data on new combination therapies. Curr Opin Oncol. 2013;25: 166-172.

91. Topalian SL, Hodi FS, Brahmer JR, Gettinger SN, Smith DC, McDermott DF, et al. Safety, activity, and immune correlates of anti-PD-1 antibody in cancer. N Engl J Med. 2012;366: 2443-2454.

92. Shah DJ, Dronca RS. Latest advances in chemotherapeutic, targeted, and immune approaches in the treatment of metastatic melanoma. Mayo Clin Proc. 2014;89: 504-519.

93. Perez-Gracia JL, Labiano S, Rodriguez-Ruiz ME, Sanmamed MF, Melero I. Orchestrating immune check-point blockade for cancer immunotherapy in combinations. Curr Opin Immunol. 2014;27: 89-97.

94. Brahmer JR, Tykodi SS, Chow LQ, Hwu WJ, Topalian SL, Hwu P, et al. Safety and activity of anti-PD-L1 antibody in patients with advanced cancer. N Engl J Med. 2012;366: 2455-2465.

95. Curran MA, Montalvo W, Yagita H, Allison JP. PD-1 and CTLA-4 combination blockade expands infiltrating $\mathrm{T}$ cells and reduces regulatory $\mathrm{T}$ and myeloid cells within B16 melanoma tumors. Proc Natl Acad Sci U S A. 2010;107: 4275-4280. 
96. Wolchok JD, Kluger H, Callahan MK, Postow MA, Rizvi NA, Lesokhin AM, et al. Nivolumab plus ipilimumab in advanced melanoma. N Engl J Med. 2013;369: 122-133.

97. Kudchadkar RR, Gibney GT, Weber J, Chen A, Smith K, Merek S. A phase IB study of ipilimumab with peginterferon alfa- $2 \mathrm{~b}$ in patients with unresectable melanoma. Journal of Clinical Oncology. 2013;31.

98. Hodi FS, Lee SJ, McDermott DF, Rao UNM, Butterfield LH, Tarhini AA, et al. Multicenter, randomized phase II trial of GM-CSF (GM) plus ipilimumab (Ipi) versus ipi alone in metastatic melanoma: E1608. Journal of Clinical Oncology. 2013;31.

99. Kwong ML, Neyns B, Yang JC. Adoptive T-cell transfer therapy and oncogenetargeted therapy for melanoma: the search for synergy. Clin Cancer Res. 2013;19: 5292-5299.

100. Wilmott JS, Long GV, Howle JR, Haydu LE, Sharma RN, Thompson JF, et al. Selective BRAF Inhibitors Induce Marked T-cell Infiltration into Human Metastatic Melanoma. Clinical Cancer Research. 2012;18: 1386-1394.

101. Donia M, Fagone P, Nicoletti F, Andersen RS, Hogdall E, Straten PT, et al. BRAF inhibition improves tumor recognition by the immune system: Potential implications for combinatorial therapies against melanoma involving adoptive Tcell transfer. Oncoimmunology. 2012;1: 1476-1483.

102. Liu C, Peng W, Xu C, Lou Y, Zhang M, Wargo JA, et al. BRAF inhibition increases tumor infiltration by $\mathrm{T}$ cells and enhances the antitumor activity of adoptive immunotherapy in mice. Clin Cancer Res. 2013;19: 393-403.

103. Jiang X, Zhou J, Giobbie-Hurder A, Wargo J, Hodi FS. The activation of MAPK in melanoma cells resistant to BRAF inhibition promotes PD-L1 expression that is reversible by MEK and PI3K inhibition. Clin Cancer Res. 2013;19: 598-609.

104. Vella LJ, Pasam A, Dimopoulos N, Andrews M, Knights A, Puaux AL, et al. MEK inhibition, alone or in combination with BRAF inhibition, affects multiple functions of isolated normal human lymphocytes and dendritic cells. Cancer Immunol Res. 2014;2: 351-360.

105. Ribas A, Hodi FS, Callahan M, Konto C, Wolchok J. Hepatotoxicity with combination of vemurafenib and ipilimumab. N Engl J Med. 2013;368: 13651366.

106. Lee JT, Li L, Brafford PA, van den Eijnden M, Halloran MB, Sproesser K, et al. PLX4032, a potent inhibitor of the B-Raf V600E oncogene, selectively inhibits V600E-positive melanomas. Pigment Cell Melanoma Res. 2010;23: 820-827.

107. Yang H, Higgins B, Kolinsky K, Packman K, Go Z, Iyer R, et al. RG7204 (PLX4032), a selective BRAFV600E inhibitor, displays potent antitumor activity in preclinical melanoma models. Cancer Res. 2010;70: 5518-5527.

108. Yang H, Higgins B, Kolinsky K, Packman K, Bradley WD, Lee RJ, et al. Antitumor activity of BRAF inhibitor vemurafenib in preclinical models of BRAF-mutant colorectal cancer. Cancer Res. 2012;72: 779-789.

109. Little AS, Smith PD, Cook SJ. Mechanisms of acquired resistance to ERK1/2 pathway inhibitors. Oncogene. 2012. 
110. Bollag G, Hirth P, Tsai J, Zhang J, Ibrahim PN, Cho H, et al. Clinical efficacy of a RAF inhibitor needs broad target blockade in BRAF-mutant melanoma. Nature. 2010;467: 596-599.

111. Flaherty KT. Targeting metastatic melanoma. Annu Rev Med. 2012;63: 171-183.

112. Shi H, Moriceau G, Kong X, Lee MK, Lee H, Koya RC, et al. Melanoma wholeexome sequencing identifies (V600E)B-RAF amplification-mediated acquired BRAF inhibitor resistance. Nat Commun. 2012;3: 724.

113. Montagut C, Sharma SV, Shioda T, McDermott U, Ulman M, Ulkus LE, et al. Elevated CRAF as a potential mechanism of acquired resistance to BRAF inhibition in melanoma. Cancer Res. 2008;68: 4853-4861.

114. Nazarian R, Shi H, Wang Q, Kong X, Koya RC, Lee H, et al. Melanomas acquire resistance to B-RAF(V600E) inhibition by RTK or N-RAS upregulation. Nature. 2010;468: 973-977.

115. Girotti MR, Pedersen M, Sanchez-Laorden B, Viros A, Turajlic S, NiculescuDuvaz D, et al. Inhibiting EGF Receptor or SRC Family Kinase Signaling Overcomes BRAF Inhibitor Resistance in Melanoma. Cancer Discov. 2013;3: 158-167.

116. Whittaker S, Kirk R, Hayward R, Zambon A, Viros A, Cantarino N, et al. Gatekeeper mutations mediate resistance to BRAF-targeted therapies. Sci Transl Med. 2010;2: 35ra41.

117. Balzano D, Santaguida S, Musacchio A, Villa F. A general framework for inhibitor resistance in protein kinases. Chem Biol. 2011;18: 966-975.

118. Sierra JR, Cepero V, Giordano S. Molecular mechanisms of acquired resistance to tyrosine kinase targeted therapy. Mol Cancer. 2010;9: 75.

119. Wilson TR, Fridlyand J, Yan Y, Penuel E, Burton L, Chan E, et al. Widespread potential for growth-factor-driven resistance to anticancer kinase inhibitors. Nature. 2012;487: 505-509.

120. Poulikakos PI, Persaud Y, Janakiraman M, Kong X, Ng C, Moriceau G, et al. RAF inhibitor resistance is mediated by dimerization of aberrantly spliced BRAF(V600E). Nature. 2011;480: 387-390.

121. Carrick S, Parker S, Wilcken N, Ghersi D, Marzo M, Simes J. Single agent versus combination chemotherapy for metastatic breast cancer. Cochrane Database Syst Rev. 2005: CD003372.

122. Fassnacht M, Terzolo M, Allolio B, Baudin E, Haak H, Berruti A, et al. Combination chemotherapy in advanced adrenocortical carcinoma. N Engl J Med. 2012;366: 2189-2197.

123. Pannu V, Karna P, Sajja HK, Shukla D, Aneja R. Synergistic antimicrotubule therapy for prostate cancer. Biochem Pharmacol. 2011;81: 478-487.

124. Patel SP, Lazar AJ, Papadopoulos NE, Liu P, Infante JR, Glass MR, et al. Clinical responses to selumetinib (AZD6244; ARRY-142886)-based combination therapy stratified by gene mutations in patients with metastatic melanoma. Cancer. 2012.

125. Chen J, Li CM, Wang J, Ahn S, Wang Z, Lu Y, et al. Synthesis and antiproliferative activity of novel 2-aryl-4-benzoyl-imidazole derivatives targeting tubulin polymerization. Bioorg Med Chem. 2011;19: 4782-4795. 
126. Chen J, Wang Z, Li CM, Lu Y, Vaddady PK, Meibohm B, et al. Discovery of novel 2-aryl-4-benzoyl-imidazoles targeting the colchicines binding site in tubulin as potential anticancer agents. J Med Chem. 2010;53: 7414-7427.

127. Chen J, Ahn S, Wang J, Lu Y, Dalton JT, Miller DD, et al. Discovery of novel 2aryl-4-benzoyl-imidazole (ABI-III) analogues targeting tubulin polymerization as antiproliferative agents. J Med Chem. 2012;55: 7285-7289.

128. Li CM, Lu Y, Chen J, Costello TA, Narayanan R, Dalton MN, et al. Orally bioavailable tubulin antagonists for paclitaxel-refractory cancer. Pharm Res. 2012;29: 3053-3063.

129. Wang Z, Chen J, Wang J, Ahn S, Li CM, Lu Y, et al. Novel tubulin polymerization inhibitors overcome multidrug resistance and reduce melanoma lung metastasis. Pharm Res. 2012;29: 3040-3052.

130. Chou TC. Drug combination studies and their synergy quantification using the Chou-Talalay method. Cancer Res. 2010;70: 440-446.

131. Villanueva J, Infante JR, Krepler C, Reyes-Uribe P, Samanta M, Chen HY, et al. Concurrent MEK2 mutation and BRAF amplification confer resistance to BRAF and MEK inhibitors in melanoma. Cell Rep. 2013;4: 1090-1099.

132. Bollag G, Tsai J, Zhang J, Zhang C, Ibrahim P, Nolop K, et al. Vemurafenib: the first drug approved for BRAF-mutant cancer. Nat Rev Drug Discov. 2012;11: 873-886.

133. Paraiso KH, Haarberg HE, Wood E, Rebecca VW, Chen YA, Xiang Y, et al. The HSP90 inhibitor XL888 overcomes BRAF inhibitor resistance mediated through diverse mechanisms. Clin Cancer Res. 2012;18: 2502-2514.

134. Koya RC, Mok S, Otte N, Blacketor KJ, Comin-Anduix B, Tumeh PC, et al. BRAF inhibitor vemurafenib improves the antitumor activity of adoptive cell immunotherapy. Cancer Res. 2012;72: 3928-3937.

135. Bartholomeusz C, Gonzalez-Angulo AM. Targeting the PI3K signaling pathway in cancer therapy. Expert Opin Ther Targets. 2012;16: 121-130.

136. Liu R, Liu D, Xing M. The Akt inhibitor MK2206 synergizes, but perifosine antagonizes, the BRAF(V600E) inhibitor PLX4032 and the MEK1/2 inhibitor AZD6244 in the inhibition of thyroid cancer cells. J Clin Endocrinol Metab. 2012;97: E173-182.

137. Krishnegowda G, Prakasha Gowda AS, Tagaram HR, Carroll KF, Irby RB, Sharma AK, et al. Synthesis and biological evaluation of a novel class of isatin analogs as dual inhibitors of tubulin polymerization and Akt pathway. Bioorg Med Chem. 2011;19: 6006-6014.

138. Viola G, Bortolozzi R, Hamel E, Moro S, Brun P, Castagliuolo I, et al. MG-2477, a new tubulin inhibitor, induces autophagy through inhibition of the Akt/mTOR pathway and delayed apoptosis in A549 cells. Biochem Pharmacol. 2012;83: 1626.

139. Zhang C, Yang N, Yang CH, Ding HS, Luo C, Zhang Y, et al. S9, a novel anticancer agent, exerts its anti-proliferative activity by interfering with both PI3K-Akt-mTOR signaling and microtubule cytoskeleton. PLoS One. 2009;4: e4881.

140. Bhalla KN. Microtubule-targeted anticancer agents and apoptosis. Oncogene. 2003;22: 9075-9086. 
141. Haass NK, Sproesser K, Nguyen TK, Contractor R, Medina CA, Nathanson KL, et al. The mitogen-activated protein/extracellular signal-regulated kinase kinase inhibitor AZD6244 (ARRY-142886) induces growth arrest in melanoma cells and tumor regression when combined with docetaxel. Clin Cancer Res. 2008;14: 230239.

142. Ambrosini G, Adida C, Altieri DC. A novel anti-apoptosis gene, survivin, expressed in cancer and lymphoma. Nat Med. 1997;3: 917-921.

143. Ryan BM, O'Donovan N, Duffy MJ. Survivin: a new target for anti-cancer therapy. Cancer Treat Rev. 2009;35: 553-562.

144. Sun C, Nettesheim D, Liu Z, Olejniczak ET. Solution structure of human survivin and its binding interface with Smac/Diablo. Biochemistry. 2005;44: 11-17.

145. Wendt MD, Sun C, Kunzer A, Sauer D, Sarris K, Hoff E, et al. Discovery of a novel small molecule binding site of human survivin. Bioorg Med Chem Lett. 2007; 17: 3122-3129.

146. Altieri DC. Survivin, cancer networks and pathway-directed drug discovery. Nat Rev Cancer. 2008;8: 61-70.

147. Tamm I, Wang Y, Sausville E, Scudiero DA, Vigna N, Oltersdorf T, et al. IAPfamily protein survivin inhibits caspase activity and apoptosis induced by Fas (CD95), Bax, caspases, and anticancer drugs. Cancer Res. 1998;58: 5315-5320.

148. Satoh K, Kaneko K, Hirota M, Masamune A, Satoh A, Shimosegawa T. Expression of survivin is correlated with cancer cell apoptosis and is involved in the development of human pancreatic duct cell tumors. Cancer. 2001;92: 271-278.

149. Kawasaki H, Toyoda M, Shinohara H, Okuda J, Watanabe I, Yamamoto T, et al. Expression of survivin correlates with apoptosis, proliferation, and angiogenesis during human colorectal tumorigenesis. Cancer. 2001;91: 2026-2032.

150. Altieri DC. Targeting survivin in cancer. Cancer Lett. 2013;332: 225-228.

151. Bates DJ, Lewis LD. Manipulating the apoptotic pathway: potential therapeutics for cancer patients. Br J Clin Pharmacol. 2013;76: 381-395.

152. Athanasoula K, Gogas H, Polonifi K, Vaiopoulos AG, Polyzos A, Mantzourani M. Survivin beyond physiology: orchestration of multistep carcinogenesis and therapeutic potentials. Cancer Lett. 2014;347: 175-182.

153. Singh N, Subramanian K, Kanwar RK, Cheung CH, Kanwar JR. Clinical aspects for survivin: a crucial molecule for targeting drug-resistant cancers. Drug Discov Today. 2014.

154. Cheung CH, Huang CC, Tsai FY, Lee JY, Cheng SM, Chang YC, et al. Survivin biology and potential as a therapeutic target in oncology. Onco Targets Ther. 2013;6: 1453-1462.

155. Coumar MS, Tsai FY, Kanwar JR, Sarvagalla S, Cheung CH. Treat cancers by targeting survivin: just a dream or future reality? Cancer Treat Rev. 2013;39: 802811.

156. Pennati M, Folini M, Zaffaroni N. Targeting survivin in cancer therapy. Expert Opin Ther Targets. 2008;12: 463-476.

157. Mamori S, Asakura T, Ohkawa K, Tajiri H. Survivin expression in early hepatocellular carcinoma and post-treatment with anti-cancer drug under hypoxic culture condition. World J Gastroenterol. 2007;13: 5306-5311. 
158. Fortugno P, Beltrami E, Plescia J, Fontana J, Pradhan D, Marchisio PC, et al. Regulation of survivin function by Hsp90. Proc Natl Acad Sci U S A. 2003;100: 13791-13796.

159. Cheung CH, Chen HH, Cheng LT, Lyu KW, Kanwar JR, Chang JY. Targeting Hsp90 with small molecule inhibitors induces the over-expression of the antiapoptotic molecule, survivin, in human A549, HONE-1 and HT-29 cancer cells. Mol Cancer. 2010;9: 77.

160. Peng XH, Karna P, Cao Z, Jiang BH, Zhou M, Yang L. Cross-talk between epidermal growth factor receptor and hypoxia-inducible factor-1alpha signal pathways increases resistance to apoptosis by up-regulating survivin gene expression. J Biol Chem. 2006;281: 25903-25914.

161. Asanuma H, Torigoe T, Kamiguchi K, Hirohashi Y, Ohmura T, Hirata K, et al. Survivin expression is regulated by coexpression of human epidermal growth factor receptor 2 and epidermal growth factor receptor via phosphatidylinositol 3kinase/AKT signaling pathway in breast cancer cells. Cancer Res. 2005;65: 11018-11025.

162. Carter BZ, Mak DH, Schober WD, Cabreira-Hansen M, Beran M, McQueen T, et al. Regulation of survivin expression through Bcr-Abl/MAPK cascade: targeting survivin overcomes imatinib resistance and increases imatinib sensitivity in imatinib-responsive CML cells. Blood. 2006;107: 1555-1563.

163. Okuya M, Kurosawa H, Kikuchi J, Furukawa Y, Matsui H, Aki D, et al. Upregulation of survivin by the E2A-HLF chimera is indispensable for the survival of $\mathrm{t}(17 ; 19)$-positive leukemia cells. J Biol Chem. 2010;285: 1850-1860.

164. Sommer KW, Rodgarkia-Dara CJ, Schreiner C, Holzmann K, Krupitza G, Cerni C. Oncogenic c-H-ras deregulates survivin expression: an improvement for survival. FEBS Lett. 2007;581: 4921-4926.

165. Nabilsi NH, Broaddus RR, Loose DS. DNA methylation inhibits p53-mediated survivin repression. Oncogene. 2009;28: 2046-2050.

166. Erkanli S, Kayaselcuk F, Kuscu E, Bagis T, Bolat F, Haberal A, et al. Expression of survivin, PTEN and p27 in normal, hyperplastic, and carcinomatous endometrium. Int J Gynecol Cancer. 2006;16: 1412-1418.

167. Guha M, Plescia J, Leav I, Li J, Languino LR, Altieri DC. Endogenous tumor suppression mediated by PTEN involves survivin gene silencing. Cancer Res. 2009;69: 4954-4958.

168. $\mathrm{Ku}$ JH, Godoy G, Amiel GE, Lerner SP. Urine survivin as a diagnostic biomarker for bladder cancer: a systematic review. BJU Int. 2012;110: 630-636.

169. Santa Cruz Guindalini R, Mathias Machado MC, Garicochea B. Monitoring survivin expression in cancer: implications for prognosis and therapy. Mol Diagn Ther. 2013;17: 331-342.

170. Zhao J, Tenev T, Martins LM, Downward J, Lemoine NR. The ubiquitinproteasome pathway regulates survivin degradation in a cell cycle-dependent manner. J Cell Sci. 2000;113 Pt 23: 4363-4371.

171. White-Gilbertson S, Kurtz DT, Voelkel-Johnson C. The role of protein synthesis in cell cycling and cancer. Mol Oncol. 2009;3: 402-408. 
172. Arora V, Cheung HH, Plenchette S, Micali OC, Liston P, Korneluk RG. Degradation of survivin by the X-linked inhibitor of apoptosis (XIAP)-XAF1 complex. J Biol Chem. 2007;282: 26202-26209.

173. Esteve PO, Chin HG, Pradhan S. Human maintenance DNA (cytosine-5)methyltransferase and p53 modulate expression of p53-repressed promoters. Proc Natl Acad Sci U S A. 2005;102: 1000-1005.

174. Wang Z, Fukuda S, Pelus LM. Survivin regulates the p53 tumor suppressor gene family. Oncogene. 2004;23: 8146-8153.

175. Vegran F, Boidot R, Oudin C, Defrain C, Rebucci M, Lizard-Nacol S. Association of p53 gene alterations with the expression of antiapoptotic survivin splice variants in breast cancer. Oncogene. 2007;26: 290-297.

176. Xu R, Zhang P, Huang J, Ge S, Lu J, Qian G. Sp1 and Sp3 regulate basal transcription of the survivin gene. Biochem Biophys Res Commun. 2007;356: 286-292.

177. Chen Y, Wang X, Li W, Zhang H, Zhao C, Li Y, et al. Sp1 upregulates survivin expression in adenocarcinoma of lung cell line A549. Anat Rec (Hoboken). 2011;294: 774-780.

178. Obexer P, Hagenbuchner J, Unterkircher T, Sachsenmaier N, Seifarth C, Bock G, et al. Repression of BIRC5/survivin by FOXO3/FKHRL1 sensitizes human neuroblastoma cells to DNA damage-induced apoptosis. Mol Biol Cell. 2009;20: 2041-2048.

179. Hagenbuchner J, Kuznetsov AV, Obexer P, Ausserlechner MJ. BIRC5/Survivin enhances aerobic glycolysis and drug resistance by altered regulation of the mitochondrial fusion/fission machinery. Oncogene. 2013;32: 4748-4757.

180. Kramer OH, Baus D, Knauer SK, Stein S, Jager E, Stauber RH, et al. Acetylation of Stat1 modulates NF-kappaB activity. Genes Dev. 2006;20: 473-485.

181. Aggarwal BB, Kunnumakkara AB, Harikumar KB, Gupta SR, Tharakan ST, Koca C, et al. Signal transducer and activator of transcription-3, inflammation, and cancer: how intimate is the relationship? Ann N Y Acad Sci. 2009;1171: 5976.

182. Clevers H, Nusse R. Wnt/beta-catenin signaling and disease. Cell. 2012;149: 1192-1205.

183. Vaira V, Lee CW, Goel HL, Bosari S, Languino LR, Altieri DC. Regulation of survivin expression by IGF-1/mTOR signaling. Oncogene. 2007;26: 2678-2684.

184. O'Connor DS, Grossman D, Plescia J, Li F, Zhang H, Villa A, et al. Regulation of apoptosis at cell division by p34cdc2 phosphorylation of survivin. Proc Natl Acad Sci U S A. 2000;97: 13103-13107.

185. Dohi T, Xia F, Altieri DC. Compartmentalized phosphorylation of IAP by protein kinase A regulates cytoprotection. Mol Cell. 2007;27: 17-28.

186. Chu Y, Yao PY, Wang W, Wang D, Wang Z, Zhang L, et al. Aurora B kinase activation requires survivin priming phosphorylation by PLK1. J Mol Cell Biol. 2011;3: 260-267.

187. Wang H, Holloway MP, Ma L, Cooper ZA, Riolo M, Samkari A, et al. Acetylation directs survivin nuclear localization to repress STAT3 oncogenic activity. J Biol Chem. 2010;285: 36129-36137. 
188. Riolo MT, Cooper ZA, Holloway MP, Cheng Y, Bianchi C, Yakirevich E, et al. Histone deacetylase 6 (HDAC6) deacetylates survivin for its nuclear export in breast cancer. J Biol Chem. 2012;287: 10885-10893.

189. Lens SM, Vader G, Medema RH. The case for Survivin as mitotic regulator. Curr Opin Cell Biol. 2006;18: 616-622.

190. Adams RR, Carmena M, Earnshaw WC. Chromosomal passengers and the (aurora) ABCs of mitosis. Trends Cell Biol. 2001;11: 49-54.

191. Shin S, Sung BJ, Cho YS, Kim HJ, Ha NC, Hwang JI, et al. An anti-apoptotic protein human survivin is a direct inhibitor of caspase-3 and -7. Biochemistry. 2001;40: 1117-1123.

192. Song Z, Yao X, Wu M. Direct interaction between survivin and Smac/DIABLO is essential for the anti-apoptotic activity of survivin during taxol-induced apoptosis. J Biol Chem. 2003;278: 23130-23140.

193. Oikawa T, Unno Y, Matsuno K, Sawada J, Ogo N, Tanaka K, et al. Identification of a small-molecule inhibitor of the interaction between Survivin and Smac/DIABLO. Biochem Biophys Res Commun. 2010;393: 253-258.

194. Du J, Kelly AE, Funabiki H, Patel DJ. Structural basis for recognition of H3T3ph and Smac/DIABLO N-terminal peptides by human Survivin. Structure. 2012;20: 185-195.

195. Croci DO, Cogno IS, Vittar NB, Salvatierra E, Trajtenberg F, Podhajcer OL, et al. Silencing survivin gene expression promotes apoptosis of human breast cancer cells through a caspase-independent pathway. J Cell Biochem. 2008;105: 381390.

196. Kelly AE, Sampath SC, Maniar TA, Woo EM, Chait BT, Funabiki H. Chromosomal enrichment and activation of the aurora B pathway are coupled to spatially regulate spindle assembly. Dev Cell. 2007;12: 31-43.

197. Kelly AE, Ghenoiu C, Xue JZ, Zierhut C, Kimura H, Funabiki H. Survivin reads phosphorylated histone $\mathrm{H} 3$ threonine 3 to activate the mitotic kinase Aurora B. Science. 2010;330: 235-239.

198. Jiang G, Ren B, Xu L, Song S, Zhu C, Ye F. Survivin may enhance DNA doublestrand break repair capability by up-regulating $\mathrm{Ku} 70$ in human $\mathrm{KB}$ cells. Anticancer Res. 2009;29: 223-228.

199. Hu S, Qu Y, Xu X, Xu Q, Geng J, Xu J. Nuclear survivin and its relationship to DNA damage repair genes in non-small cell lung cancer investigated using tissue array. PLoS One. 2013;8: e74161.

200. Chang YJ, Li LT, Chen HA, Hung CS, Wei PL. Silencing survivin activates autophagy as an alternative survival pathway in HCC cells. Tumour Biol. 2014;35: 9957-9966.

201. Niu TK, Cheng Y, Ren X, Yang JM. Interaction of Beclin 1 with survivin regulates sensitivity of human glioma cells to TRAIL-induced apoptosis. FEBS Lett. 2010;584: 3519-3524.

202. Rauch A, Hennig D, Schafer C, Wirth M, Marx C, Heinzel T, et al. Survivin and YM155: how faithful is the liaison? Biochim Biophys Acta. 2014;1845: 202-220. 
203. Giaccone G, Zatloukal P, Roubec J, Floor K, Musil J, Kuta M, et al. Multicenter phase II trial of YM155, a small-molecule suppressor of survivin, in patients with advanced, refractory, non-small-cell lung cancer. J Clin Oncol. 2009;27: 44814486.

204. Nakahara T, Yamanaka K, Hatakeyama S, Kita A, Takeuchi M, Kinoyama I, et al. YM155, a novel survivin suppressant, enhances taxane-induced apoptosis and tumor regression in a human Calu 6 lung cancer xenograft model. Anticancer Drugs. 2011;22: 454-462.

205. Satoh T, Okamoto I, Miyazaki M, Morinaga R, Tsuya A, Hasegawa Y, et al. Phase I study of YM155, a novel survivin suppressant, in patients with advanced solid tumors. Clin Cancer Res. 2009;15: 3872-3880.

206. Yamanaka K, Nakahara T, Yamauchi T, Kita A, Takeuchi M, Kiyonaga F, et al. Antitumor activity of YM155, a selective small-molecule survivin suppressant, alone and in combination with docetaxel in human malignant melanoma models. Clin Cancer Res. 2011;17: 5423-5431.

207. Nakahara T, Kita A, Yamanaka K, Mori M, Amino N, Takeuchi M, et al. YM155, a novel small-molecule survivin suppressant, induces regression of established human hormone-refractory prostate tumor xenografts. Cancer Res. 2007;67: 8014-8021.

208. Ling X, Cao S, Cheng Q, Keefe JT, Rustum YM, Li F. A novel small molecule FL118 that selectively inhibits survivin, Mcl-1, XIAP and cIAP2 in a p53independent manner, shows superior antitumor activity. PLoS One. 2012;7: e45571.

209. Zhao J, Ling X, Cao S, Liu X, Wan S, Jiang T, et al. Antitumor activity of FL118, a survivin, Mcl-1, XIAP, and cIAP2 selective inhibitor, is highly dependent on its primary structure and steric configuration. Mol Pharm. 2014;11: 457-467.

210. Smolewski P. Terameprocol, a novel site-specific transcription inhibitor with anticancer activity. IDrugs. 2008;11: 204-214.

211. Khanna N, Dalby R, Tan M, Arnold S, Stern J, Frazer N. Phase I/II clinical safety studies of terameprocol vaginal ointment. Gynecol Oncol. 2007;107: 554-562.

212. Grossman SA, Ye X, Peereboom D, Rosenfeld MR, Mikkelsen T, Supko JG, et al. Phase I study of terameprocol in patients with recurrent high-grade glioma. Neuro Oncol. 2012;14: 511-517.

213. Gyurkocza B, Plescia J, Raskett CM, Garlick DS, Lowry PA, Carter BZ, et al. Antileukemic activity of shepherdin and molecular diversity of hsp90 inhibitors. J Natl Cancer Inst. 2006;98: 1068-1077.

214. Plescia J, Salz W, Xia F, Pennati M, Zaffaroni N, Daidone MG, et al. Rational design of shepherdin, a novel anticancer agent. Cancer Cell. 2005;7: 457-468.

215. Meli M, Pennati M, Curto M, Daidone MG, Plescia J, Toba S, et al. Smallmolecule targeting of heat shock protein 90 chaperone function: rational identification of a new anticancer lead. J Med Chem. 2006;49: 7721-7730.

216. Wall NR, O'Connor DS, Plescia J, Pommier Y, Altieri DC. Suppression of survivin phosphorylation on Thr34 by flavopiridol enhances tumor cell apoptosis. Cancer Res. 2003;63: 230-235. 
217. Pennati M, Campbell AJ, Curto M, Binda M, Cheng Y, Wang LZ, et al.

Potentiation of paclitaxel-induced apoptosis by the novel cyclin-dependent kinase inhibitor NU6140: a possible role for survivin down-regulation. Mol Cancer Ther. 2005;4: 1328-1337.

218. Villerbu N, Gaben AM, Redeuilh G, Mester J. Cellular effects of purvalanol A: a specific inhibitor of cyclin-dependent kinase activities. Int J Cancer. 2002;97: 761-769.

219. Iizuka D, Ogura A, Kuwabara M, Inanami O. Purvalanol A induces apoptosis and downregulation of antiapoptotic proteins through abrogation of phosphorylation of JAK2/STAT3 and RNA polymerase II. Anticancer Drugs. 2008;19: 565-572.

220. Lee HG, Baek JW, Shin SJ, Kwon SH, Cha SD, Park WJ, et al. Antitumor effects of flavopiridol on human uterine leiomyoma in vitro and in a xenograft model. Reprod Sci. 2014;21: 1153-1160.

221. Jain SK, Bharate SB, Vishwakarma RA. Cyclin-dependent kinase inhibition by flavoalkaloids. Mini Rev Med Chem. 2012;12: 632-649.

222. Carrasco RA, Stamm NB, Marcusson E, Sandusky G, Iversen P, Patel BK. Antisense inhibition of survivin expression as a cancer therapeutic. Mol Cancer Ther. 2011;10: 221-232.

223. Talbot DC, Ranson M, Davies J, Lahn M, Callies S, Andre V, et al. Tumor survivin is downregulated by the antisense oligonucleotide LY2181308: a proofof-concept, first-in-human dose study. Clin Cancer Res. 2010;16: 6150-6158.

224. Wiechno P, Somer BG, Mellado B, Chlosta PL, Cervera Grau JM, Castellano D, et al. A randomised phase 2 study combining LY2181308 sodium (survivin antisense oligonucleotide) with first-line docetaxel/prednisone in patients with castration-resistant prostate cancer. Eur Urol. 2014;65: 516-520.

225. Hansen JB, Fisker N, Westergaard M, Kjaerulff LS, Hansen HF, Thrue CA, et al. SPC3042: a proapoptotic survivin inhibitor. Mol Cancer Ther. 2008;7: 27362745.

226. Sapra P, Wang M, Bandaru R, Zhao H, Greenberger LM, Horak ID. Downmodulation of survivin expression and inhibition of tumor growth in vivo by EZN-3042, a locked nucleic acid antisense oligonucleotide. Nucleosides Nucleotides Nucleic Acids. 2010;29: 97-112.

227. Raetz EA, Morrison D, Romanos-Sirakis E, Gaynon P, Sposto R, Bhojwani D, et al. A phase I study of EZN-3042, a novel survivin messenger ribonucleic acid (mRNA) antagonist, administered in combination with chemotherapy in children with relapsed acute lymphoblastic leukemia (ALL): a report from the therapeutic advances in childhood leukemia and lymphoma (TACL) consortium. J Pediatr Hematol Oncol. 2014;36: 458-463.

228. Aspe JR, Wall NR. Survivin-T34A: molecular mechanism and therapeutic potential. Onco Targets Ther. 2010;3: 247-254.

229. Grossman D, Kim PJ, Schechner JS, Altieri DC. Inhibition of melanoma tumor growth in vivo by survivin targeting. Proc Natl Acad Sci U S A. 2001;98: 635640.

230. Yan H, Thomas J, Liu T, Raj D, London N, Tandeski T, et al. Induction of melanoma cell apoptosis and inhibition of tumor growth using a cell-permeable Survivin antagonist. Oncogene. 2006;25: 6968-6974. 
231. Kanwar JR, Shen WP, Kanwar RK, Berg RW, Krissansen GW. Effects of survivin antagonists on growth of established tumors and B7-1 immunogene therapy. J Natl Cancer Inst. 2001;93: 1541-1552.

232. Baratchi S, Kanwar RK, Kanwar JR. Novel survivin mutant protects differentiated SK-N-SH human neuroblastoma cells from activated T-cell neurotoxicity. J Neuroimmunol. 2011;233: 18-28.

233. Baratchi S, Kanwar RK, Cheung CH, Kanwar JR. Proliferative and protective effects of SurR9-C84A on differentiated neural cells. J Neuroimmunol. 2010;227: 120-132.

234. Roy K, Kanwar RK, Krishnakumar S, Cheung CH, Kanwar JR. Competitive inhibition of survivin using a cell-permeable recombinant protein induces cancerspecific apoptosis in colon cancer model. Int J Nanomedicine. 2015;10: 10191043.

235. Zhang R, Wang T, Li KN, Qin WW, Chen R, Wang K, et al. A survivin double point mutant has potent inhibitory effect on the growth of hepatocellular cancer cells. Cancer Biol Ther. 2008;7: 547-554.

236. Li F, Ackermann EJ, Bennett CF, Rothermel AL, Plescia J, Tognin S, et al. Pleiotropic cell-division defects and apoptosis induced by interference with survivin function. Nat Cell Biol. 1999;1: 461-466.

237. Molckovsky A, Siu LL. First-in-class, first-in-human phase I results of targeted agents: highlights of the 2008 American society of clinical oncology meeting. J Hematol Oncol. 2008;1: 20.

238. Paduano F, Villa R, Pennati M, Folini M, Binda M, Daidone MG, et al. Silencing of survivin gene by small interfering RNAs produces supra-additive growth suppression in combination with 17-allylamino-17-demethoxygeldanamycin in human prostate cancer cells. Mol Cancer Ther. 2006;5: 179-186.

239. Zhen HN, Li LW, Zhang W, Fei Z, Shi CH, Yang TT, et al. Short hairpin RNA targeting survivin inhibits growth and angiogenesis of glioma U251 cells. Int $\mathrm{J}$ Oncol. 2007;31: 1111-1117.

240. Zaffaroni N, Daidone MG. Survivin expression and resistance to anticancer treatments: perspectives for new therapeutic interventions. Drug Resist Updat. 2002;5: 65-72.

241. Iwai M, Minematsu T, Li Q, Iwatsubo T, Usui T. Utility of P-glycoprotein and organic cation transporter 1 double-transfected LLC-PK1 cells for studying the interaction of YM155 monobromide, novel small-molecule survivin suppressant, with P-glycoprotein. Drug Metab Dispos. 2011;39: 2314-2320.

242. Guenova ML, Balatzenko GN, Nikolova VR, Spassov BV, Konstantinov SM. An anti-apoptotic pattern correlates with multidrug resistance in acute myeloid leukemia patients: a comparative study of active caspase-3, cleaved PARPs, Bcl2, Survivin and MDR1 gene. Hematology. 2010;15: 135-143.

243. Baytekin F, Tuna B, Mungan U, Aslan G, Yorukoglu K. Significance of Pglycoprotein, $\mathrm{p} 53$, and survivin expression in renal cell carcinoma. Urol Oncol. 2011;29: 502-507.

244. Imai Y, Yamagishi H, Ono Y, Ueda Y. Versatile inhibitory effects of the flavonoid-derived PI3K/Akt inhibitor, LY294002, on ATP-binding cassette transporters that characterize stem cells. Clin Transl Med. 2012;1: 24. 
245. Fulda S, Vucic D. Targeting IAP proteins for therapeutic intervention in cancer. Nat Rev Drug Discov. 2012;11: 109-124.

246. Wu H, Panakanti R, Li F, Mahato RI. XIAP gene expression protects beta-cells and human islets from apoptotic cell death. Mol Pharm. 2010;7: 1655-1666.

247. Tian F, Lee SW. X-linked inhibitor of apoptosis protein as a therapeutic target in metastatic melanoma. J Invest Dermatol. 2010;130: 2169-2172.

248. Wang S. Design of small-molecule Smac mimetics as IAP antagonists. Curr Top Microbiol Immunol. 2011;348: 89-113.

249. Wu G, Chai J, Suber TL, Wu JW, Du C, Wang X, et al. Structural basis of IAP recognition by Smac/DIABLO. Nature. 2000;408: 1008-1012.

250. de Almagro MC, Vucic D. The inhibitor of apoptosis (IAP) proteins are critical regulators of signaling pathways and targets for anti-cancer therapy. Exp Oncol. 2012;34: 200-211.

251. Flygare JA, Beresini M, Budha N, Chan H, Chan IT, Cheeti S, et al. Discovery of a potent small-molecule antagonist of inhibitor of apoptosis (IAP) proteins and clinical candidate for the treatment of cancer (GDC-0152). J Med Chem. 2012;55: 4101-4113.

252. Lu J, Bai L, Sun H, Nikolovska-Coleska Z, McEachern D, Qiu S, et al. SM-164: a novel, bivalent Smac mimetic that induces apoptosis and tumor regression by concurrent removal of the blockade of cIAP-1/2 and XIAP. Cancer Res. 2008;68: 9384-9393.

253. Nikolovska-Coleska Z, Meagher JL, Jiang S, Kawamoto SA, Gao W, Yi H, et al. Design and characterization of bivalent Smac-based peptides as antagonists of $\mathrm{XIAP}$ and development and validation of a fluorescence polarization assay for XIAP containing both BIR2 and BIR3 domains. Anal Biochem. 2008;374: 87-98.

254. Nikolovska-Coleska Z, Xu L, Hu Z, Tomita Y, Li P, Roller PP, et al. Discovery of embelin as a cell-permeable, small-molecular weight inhibitor of XIAP through structure-based computational screening of a traditional herbal medicine threedimensional structure database. J Med Chem. 2004;47: 2430-2440.

255. Peng Y, Sun H, Lu J, Liu L, Cai Q, Shen R, et al. Bivalent Smac mimetics with a diazabicyclic core as highly potent antagonists of XIAP and cIAP1/2 and novel anticancer agents. J Med Chem. 2012;55: 106-114.

256. Sun H, Nikolovska-Coleska Z, Lu J, Meagher JL, Yang CY, Qiu S, et al. Design, synthesis, and characterization of a potent, nonpeptide, cell-permeable, bivalent Smac mimetic that concurrently targets both the BIR2 and BIR3 domains in XIAP. J Am Chem Soc. 2007;129: 15279-15294.

257. Krepler C, Chunduru SK, Halloran MB, He X, Xiao M, Vultur A, et al. The novel SMAC mimetic birinapant exhibits potent activity against human melanoma cells. Clin Cancer Res. 2013;19: 1784-1794.

258. Vucic D, Franklin MC, Wallweber HJ, Das K, Eckelman BP, Shin H, et al. Engineering ML-IAP to produce an extraordinarily potent caspase 9 inhibitor: implications for Smac-dependent anti-apoptotic activity of ML-IAP. Biochem J. 2005;385: 11-20.

259. Xiao M, Ahn S, Wang J, Chen J, Miller DD, Dalton JT, et al. Discovery of 4Aryl-2-benzoyl-imidazoles as tubulin polymerization inhibitor with potent antiproliferative properties. J Med Chem. 2013;56: 3318-3329. 
260. Sastry GM, Dixon SL, Sherman W. Rapid shape-based ligand alignment and virtual screening method based on atom/feature-pair similarities and volume overlap scoring. J Chem Inf Model. 2011;51: 2455-2466.

261. Hiscutt EL, Hill DS, Martin S, Kerr R, Harbottle A, Birch-Machin M, et al. Targeting X-linked inhibitor of apoptosis protein to increase the efficacy of endoplasmic reticulum stress-induced apoptosis for melanoma therapy. J Invest Dermatol. 2010;130: 2250-2258.

262. Jane EP, Premkumar DR, DiDomenico JD, Hu B, Cheng SY, Pollack IF. YM-155 potentiates the effect of ABT-737 in malignant human glioma cells via survivin and Mcl-1 downregulation in an EGFR-dependent context. Mol Cancer Ther. 2013;12: 326-338.

263. Altieri DC. Survivin and IAP proteins in cell-death mechanisms. Biochem J. 2010;430: 199-205.

264. Kanwar JR, Kamalapuram SK, Kanwar RK. Targeting survivin in cancer: the cell-signalling perspective. Drug Discov Today. 2011;16: 485-494.

265. Pavlyukov MS, Antipova NV, Balashova MV, Vinogradova TV, Kopantzev EP, Shakhparonov MI. Survivin monomer plays an essential role in apoptosis regulation. J Biol Chem. 2011;286: 23296-23307.

266. Fukuda S, Pelus LM. Survivin, a cancer target with an emerging role in normal adult tissues. Mol Cancer Ther. 2006;5: 1087-1098.

267. McKenzie JA, Grossman D. Role of the apoptotic and mitotic regulator survivin in melanoma. Anticancer Res. 2012;32: 397-404.

268. Okamoto K, Okamoto I, Hatashita E, Kuwata K, Yamaguchi H, Kita A, et al. Overcoming erlotinib resistance in EGFR mutation-positive non-small cell lung cancer cells by targeting survivin. Mol Cancer Ther. 2012;11: 204-213.

269. Liu JJ, Huang RW, Lin DJ, Peng J, Wu XY, Lin Q, et al. Expression of survivin and bax/bcl-2 in peroxisome proliferator activated receptor-gamma ligands induces apoptosis on human myeloid leukemia cells in vitro. Ann Oncol. 2005;16: 455-459.

270. Wang Z, Azmi AS, Ahmad A, Banerjee S, Wang S, Sarkar FH, et al. TW-37, a small-molecule inhibitor of Bcl-2, inhibits cell growth and induces apoptosis in pancreatic cancer: involvement of Notch-1 signaling pathway. Cancer Res. 2009; 69: 2757-2765.

271. Lamers F, Schild L, Koster J, Versteeg R, Caron HN, Molenaar JJ. Targeted BIRC5 silencing using YM155 causes cell death in neuroblastoma cells with low ABCB1 expression. European Journal of Cancer. 2012;48: 763-771.

272. Cheng Q, Ling X, Haller A, Nakahara T, Yamanaka K, Kita A, et al. Suppression of survivin promoter activity by YM155 involves disruption of Sp1-DNA interaction in the survivin core promoter. Int J Biochem Mol Biol. 2012;3: 179197.

273. Wang J, Li W. Discovery of novel second mitochondria-derived activator of caspase mimetics as selective inhibitor of apoptosis protein inhibitors. J Pharmacol Exp Ther. 2014;349: 319-329.

274. Shoemaker RH. The NCI60 human tumour cell line anticancer drug screen. Nat Rev Cancer. 2006;6: 813-823. 
275. Lomenick B, Jung G, Wohlschlegel JA, Huang J. Target identification using drug affinity responsive target stability (DARTS). Curr Protoc Chem Biol. 2011;3: $163-180$.

276. Lomenick B, Hao R, Jonai N, Chin RM, Aghajan M, Warburton S, et al. Target identification using drug affinity responsive target stability (DARTS). Proc Natl Acad Sci U S A. 2009;106: 21984-21989.

277. Lu Y, Chen J, Wang J, Li CM, Ahn S, Barrett CM, et al. Design, synthesis, and biological evaluation of stable colchicine binding site tubulin inhibitors as potential anticancer agents. J. Med. Chem. 2014;57: 7355-7366.

278. Leonessa F, Green D, Licht T, Wright A, Wingate-Legette K, Lippman J, et al. MDA435/LCC6 and MDA435/LCC6MDR1: ascites models of human breast cancer. Br J Cancer. 1996;73: 154-161.

279. Zhang S, Morris ME. Effects of the flavonoids biochanin A, morin, phloretin, and silymarin on P-glycoprotein-mediated transport. J Pharmacol Exp Ther. 2003;304: 1258-1267.

280. Dong X, Mattingly CA, Tseng MT, Cho MJ, Liu Y, Adams VR, et al.

Doxorubicin and paclitaxel-loaded lipid-based nanoparticles overcome multidrug resistance by inhibiting P-glycoprotein and depleting ATP. Cancer Res. 2009;69: 3918-3926.

281. Uchiyama-Kokubu N, Watanabe T. Establishment and characterization of adriamycin-resistant human colorectal adenocarcinoma HCT-15 cell lines with multidrug resistance. Anticancer Drugs. 2001;12: 769-779.

282. angthong $\mathrm{S}, \mathrm{Ha} \mathrm{H}$, Teerawattananon T, Ngamrojanavanich N, Neamati N, Muangsin N. Overcoming doxorubicin-resistance in the NCI/ADR-RES model cancer cell line by novel anthracene-9,10-dione derivatives. Bioorg Med Chem Lett. 2013;23: 6156-6160. 


\section{VITA}

Jin Wang was born in Anhui Province, China in 1988. She attended the Sun Yatsen University from Year 2005 to 2009 and received a Bachelor of Science degree in pharmacy. Then she joined the graduate program in the Department of Pharmaceutical Sciences, the University of Tennessee Health Science Center, from where she will receive her Ph.D degree under the supervision of Professor Dr. Wei Li. 